Universidade de São Paulo

Instituto de Astronomia, Geofísica e Ciências Atmosféricas

Departamento de Astronomia

Julia de Carvalho Santos

\title{
Methyl acetylene in G331.512-0.103: \\ Looking at massive star formation through the lens of chemistry
}

São Paulo 

Julia de Carvalho Santos

\section{Methyl acetylene in G331.512-0.103: \\ Looking at massive star formation through the lens of chemistry}

Thesis presented to the Department of Astronomy of Instituto de Astronomia, Geofísica e Ciências Atmosféricas of Universidade de São Paulo as a partial requirement to obtain a Master of Science degree.

Corrected version. The original printed version is available at IAG/USP

Research field: Astronomy

Advisor: Prof. Dr. Jacques R. D. Lépine Co-advisor: Dr. Edgar Mendoza

São Paulo 



\section{Acknowledgements}

The pursuit of a graduate degree is never an easy journey, and I certainly did not expect it to be. However, the outbreak of a pandemic during the first year of my master's degree cannot be described as anything less than a colossal setback. From switching to having classes and meetings online, to the unrelenting uneasiness of an unprecedented and completely unpredictable global-health conjecture, this crisis has been a real challenge that will forever remain in the memories of those who survive it. Nonetheless, "life did not stop, and one had to live"(Leo Tolstoy). And so I lived, and wrote this thesis hoping that, in the future, I will be able to look back at this moment and feel a glimpse of pride.

I would like to first and foremost thank my parents, who provided me the support to pursue this arduous career path. I would also like to thank all of my friends, old and new, for the interesting conversations and, perhaps more importantly, the laughter. I want to thank Fabio, my love, who never ceased to believe in me, even when I did. Lastly, I would like to thank myself, however narcissistic it may sound. Heaven knows I struggled.

This study was financed in part by the Coordenação de Aperfeiçoamento de Pessoal de Nível Superior Brasil (CAPES)

Esta tese/dissertação foi escrita em ATEX com a classe IAGTESE, para teses e dissertações do IAG. 

"If I were not the type of atheist who believes with unflinching faith in the purposelessness of life, who believes in the unrelenting chaos of a cold universe, who believes that life is a brutal cosmic accident, who believes there is no one watching and certainly no one pulling the strings, I would believe there is someone watching and pulling the strings, and that the someone or thing that has it out for me is indeed watching, watching, always watching, damn it." Charlie Kaufman - Antkind 



\section{Resumo}

Nesta dissertação de mestrado, nós pretendemos explorar as propriedades físicas de regiões de formação estelar massiva através de seus espectros de emissão molecular, os quais têm a capacidade de prover informações importantes a respeito da fonte. Dessa forma, nós conduzimos um survey espectral de metil-acetileno $\left(\mathrm{CH}_{3} \mathrm{CCH}\right)$ no Hot Molecular Core/Outflow massivo G331.512-0.103, usando o telescópio de 12 m APEX. As nossas observações resultaram na detecção de 41 linhas nítidas e não-contaminadas de $\mathrm{CH}_{3} \mathrm{CCH}$ no intervalo de frequências entre 172 e $356 \mathrm{GHz}$. Através de uma análise assumindo o Equilíbrio Termodinâmico Local, na qual utilizamos diagramas rotacionais, determinamos $\mathrm{T}_{\text {exc }}=47.1 \pm 1.2 \mathrm{~K}, \mathrm{~N}_{\text {tot }}\left(\mathrm{CH}_{3} \mathrm{CCH}\right)=6.9 \pm 0.5 \times 10^{15} \mathrm{~cm}^{-2}$ e $X\left[\mathrm{CH}_{3} \mathrm{CCH} / \mathrm{H}_{2}\right] \approx(1.5-7.6) \times 10^{-8}$ para uma região extensa de emissão $\left(\sim 10^{\prime \prime}\right)$. Nós observamos que as intensidades relativas das linhas com $\mathrm{K}=2$ e $\mathrm{K}=3$ em um determinado $K$-ladder apresentam uma forte correlação negativa com o número quântico $\mathrm{J}$ superior da transição $(\mathrm{r}=-0.84)$. Essa observação foi analisada em conjunto com simulações dos espectros rotacionais puros de $\mathrm{CH}_{3} \mathrm{CCH}$ em diferentes temperaturas, juntamente com adaptações da técnica do diagrama rotacional. Os resultados indicaram que a emissão é caracterizada por um gradiente de temperaturas, com limites inferior e superior de $\sim 40$ e $\sim 60 \mathrm{~K}$, respectivamente. Além disso, as larguras das linhas e as velocidades dos picos apresentam, em geral, uma forte correlação com as frequências das transições, sugerindo que o gás mais quente também está associado a efeitos mais fortes de turbulência. As transições com $\mathrm{K}=0$ apresentam uma assinatura cinemática ligeiramente diferente do resto das linhas, indicando que elas podem estar traçando uma componente distinta do gás. Nós especulamos que essa componente é caracterizada por temperaturas mais baixas, e portanto tamanhos maiores. No entanto, observações com maiores resoluções angulares são necessárias para verificar estas conclusões. 



\section{Abstract}

In the present master's thesis, we aim to explore the physical properties of massive starforming regions by means of their molecular emission spectra, which can provide powerful information on the source. Thus, we conducted a spectral survey of methyl acetylene $\left(\mathrm{CH}_{3} \mathrm{CCH}\right)$ toward the Hot Molecular Core/massive outflow G331.512-0.103, using the APEX 12 m telescope. Our observations yielded the detection of 41 clear and uncontaminated rotational lines of $\mathrm{CH}_{3} \mathrm{CCH}$ in the frequency range between $172-356 \mathrm{GHz}$. Through an analysis under the Local Thermodynamic Equilibrium (LTE) assumption, by means of rotational diagrams, we determined $\mathrm{T}_{e x c}=47.1 \pm 1.2 \mathrm{~K}, \mathrm{~N}_{t o t}\left(\mathrm{CH}_{3} \mathrm{CCH}\right)=6.9 \pm 0.5 \times 10^{15} \mathrm{~cm}^{-2}$ and $X\left[\mathrm{CH}_{3} \mathrm{CCH} / \mathrm{H}_{2}\right] \approx(1.5-7.6) \times 10^{-8}$ for an extended emitting region $\left(\sim 10^{\prime \prime}\right)$. We observed that the relative intensities of the $K=2$ and $K=3$ lines within a given K-ladder are strongly negatively correlated to the transitions' upper J quantum number $(\mathrm{r}=-0.84)$. Pure rotational spectra of $\mathrm{CH}_{3} \mathrm{CCH}$ were simulated at different temperatures, in order to explore this observation. Conjointly, adaptations to the rotational diagram technique were also employed. The results indicate that the emission is characterized by a non-negligible temperature gradient with upper and lower limits of $\sim 40$ and $\sim 60 \mathrm{~K}$, respectively. Moreover, the line widths and peak velocities show an overall strong correlation with their rest frequencies, suggesting that the warmer gas is also associated with stronger turbulence effects. The $K=0$ transitions present a slightly different kinematic signature than the remainder, indicating that they might be tracing a different gas component. We speculate that this component is characterized by lower temperatures, and therefore larger sizes. However, observations with higher angular resolution are imperative to verify our conclusions. 



\section{List of Figures}

1.1 From van Dishoeck (2014). Formation and evolution of the interstellar ices.

The $\mathrm{H}_{2} \mathrm{O}$-rich ice is shown in red, while the CO-rich ice is shown in blue. .

1.2 Sketch of the three major surface reaction mechanisms. From Herbst and van Dishoeck (2009).

1.3 Figure from Sadaghiani et al. (2020), showing the different fragmentation levels of the NGC 6334 star-forming complex from the scale of the filament down to the scale of individual dense cores and protostars. The colors indicate different densities - lighter colors correspond to lower densities, whereas darker colors indicate higher densities - as well as ionized gas (in orange). Vertical and horizontal orientations correspond to the Galactic latitude and longitude coordinates, respectively. The distribution is based on observations by Russeil et al. (2013). . . . . . . . . . . . . . . . . . . . . . . . . .

1.4 Superimposed maps of the $\mathrm{CH}_{3} \mathrm{CN}(5-4)$ traced $\mathrm{HMC}$ in the high-mass starforming region IRAS $20126+4104$ (grey scale) and the $\mathrm{C}^{17} \mathrm{O}(2-1)$ traced surrounding molecular clump (contours). From Cesaroni 2005. . . . . . .

1.5 Zeroth moment maps of emission from G331, integrated in the range of the systemic velocity. The $862 \mu \mathrm{m}$ continuum emission map is also shown (bottom right panel). From Hervías-Caimapo et al. (2019). . . . . . . . . .

1.6 Schematic view of the physical model proposed for G331. Based on the models of Merello et al. (2013a); Duronea et al. (2019); Hervías-Caimapo et al. $(2019) \ldots \ldots \ldots \ldots \ldots \ldots$

1.7 Transitions between the energy levels $E_{i}$ and $E_{j}$ of a given species and the Einstein probabilities. Adapted from Wilson et al. (2009). . . . . . . . . . 42 
1.8 Sketch of the ellipsoid of inertia of $\mathrm{H}_{2} \mathrm{CO}$ adapted from Levine (1975). Each cross-section contains two principal axes. . . . . . . . . . . 44

1.9 Overview of commonly used molecular diagnostics and the respective range of physical conditions that they trace. From Genzel 1991. . . . . . . . . 47

$1.103 \mathrm{D}$ models of the molecules $\mathrm{CH}_{3} \mathrm{CCH}, \mathrm{CH}_{3} \mathrm{CN}$ and $\mathrm{CH}_{3} \mathrm{OH}$, for comparison purposes. Symmetric rotors: a) methyl acetylene $\left(\mathrm{CH}_{3} \mathrm{CCH}\right)$ and b) acetonitrile $\left(\mathrm{CH}_{3} \mathrm{CN}\right)$. Asymmetric rotor: c) methanol $\left(\mathrm{CH}_{3} \mathrm{OH}\right)$. The white, gray, blue and red spheres correspond to the $\mathrm{H}, \mathrm{C}, \mathrm{N}$ and $\mathrm{O}$ atoms, respectively. Those three molecules have been observed in G331 (Hervías-Caimapo et al., 2019), and play an important role in the inference of the physical conditions of HMCs and related sources. . . . . . . . . . . 5 50

1.11 Rotational energy levels of $\mathrm{CH}_{3} \mathrm{CCH}$. Adapted from Kuiper et al. (1984). . 52

2.1 APEX 12 m telescope. Credit: ESO/B. Tafreshi . . . . . . . . . 54

2.2 Gaussian fitting of the $\mathrm{CH}_{3} \mathrm{CCH}\left(10_{0}-9_{0}\right)$ line (green) over the observed spectrum (black). The upper and lower $\mathrm{x}$-axes are given in frequency units $(\mathrm{MHz})$ and velocity units $\left(\mathrm{km} \mathrm{s}^{-1}\right)$, respectively. The $\mathrm{y}$-axis is given in temperature units $(\mathrm{K}) \ldots \ldots \ldots \ldots \ldots \ldots$

3.1 Spectra of $\mathrm{CH}_{3} \mathrm{CCH}$ exhibiting the different K-ladders identified from $\mathrm{J}=10$ 9 to $\mathrm{J}=18-17$. For each spectrum, a linear baseline was subtracted. K-level transitions are indicated for the $\mathrm{J}=10-9 \mathrm{~K}$-ladder. . . . . . . . . . . . 64 64

3.2 Plot of $\mathrm{A}_{i j}$ versus $\mathrm{E}_{u p}$ of the observed $\mathrm{CH}_{3} \mathrm{CCH}$ lines. The transitions are grouped according to their K quantum number: blue cross-marks correspond to transitions with $K=0$, magenta squares correspond to $K=1$, green circles correspond to $\mathrm{K}=2$, red triangles correspond to $\mathrm{K}=3$ and yellow diamonds correspond to $\mathrm{K}=4 \ldots \ldots \ldots \ldots \ldots \ldots$

3.3 Upper panel: Rotational diagram of the $\mathrm{CH}_{3} \mathrm{CCH}$ lines for a source size of $5^{\prime \prime}$. Least squares fits with and without opacity corrections are shown in red and blue, respectively. Lower panel: Same as the upper panel, but for a source size of $10^{\prime \prime} \ldots \ldots \ldots \ldots \ldots \ldots$ 
3.4 Optical depth $(\tau)$ vs. $\mathrm{E}_{u}$ of the $\mathrm{CH}_{3} \mathrm{CCH}$ lines assuming a source size of $10^{\prime \prime}$. Blue cross marks represent transitions with $\mathrm{K}=0$ and $\mathrm{K}=1$, red circles represent transitions with $\mathrm{K}=2$ and $\mathrm{K}=3$, and yellow triangles represent transitions with $\mathrm{K}=4$. Linear fits to the points are shown for each group of K quantum numbers, to guide the eye. . . . . . . . . . . . . 69 69

3.5 Spectra of $\mathrm{CH}_{3} \mathrm{CCH}$ observed toward G331. Upper pannels: J=10-9, 170.87$170.91 \mathrm{GHz}$; Lower pannels: J=18-17, 307.52-307.59 GHz. Left pannels: LTE models simulated for a compact emission $\left(5^{\prime \prime}\right)$. Right pannels: LTE models simulated for an extended emission $\left(10^{\prime \prime}\right)$. For all panels, the black and red spectra correspond to the observation and the LTE model, respectively. The dashed line indicates the systemic velocity of the source. The abscissas of all spectra were adjusted to the systemic velocity of the $K=0$

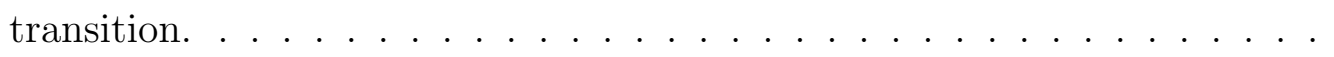

3.6 Observed spectra of the detected $\mathrm{CH}_{3} \mathrm{CCH}$ lines (black), superimposed by the LTE models (red). The dashed line indicates the systemic velocity of the source. The abscissas of all spectra were adjusted to the systemic velocity of the $\mathrm{K}=0$ transition.

3.7 Experimental (red, upper panel) and simulated (black, lower panel) pure rotational spectra of $\mathrm{CH}_{3} \mathrm{CCH}$ at $300 \mathrm{~K}$. The inset shows the $\mathrm{J}=10-9 \mathrm{~K}-$ ladder

3.8 $\mathrm{J}=10_{K^{-}} 9_{K}$ K-ladder of $\mathrm{CH}_{3} \mathrm{CCH}$ simulated at $10 \mathrm{~K}$ (upper-left panel), 40 K (upper-right panel), $70 \mathrm{~K}$ (lower-left panel) and $100 \mathrm{~K}$ (lower-right panel). 75

3.9 Intensity ratios $\mathrm{I}[\mathrm{K}=\mathrm{n}] / \mathrm{I}[\mathrm{K}=0]$ versus temperature for transitions within a given K-ladder. Purple squares represent ratios with $n=1$, green circles represent ratios with $n=2$, and red triangles represent ratios with $n=3$. Solid and dashed lines correspond to, respectively, the $\mathrm{J}=10-9$ and $\mathrm{J}=18-17$ K-ladders. The red and blue regions are discussed in 3.3 .4 . . . . . . . .

3.10 Superposition of the $K=2$ and $K=3$ lines of the $J=10-9$ (red) and $K=18-17$ (blue) K-ladders, evincing the intensity inversion of the peaks. The abscissa is adjusted to the systemic velocity of the $K=2$ lines. 
3.11 Ratios of the areas for $\mathrm{K}=2$ and $\mathrm{K}=3$ lines versus the upper $\mathrm{J}$ value of the transition. Areas and uncertainties were obtained through gaussian fittings of the observed data using CLASS. . . . . . . . . . 78

3.12 Upper panel: Modified rotational diagram of the $\mathrm{CH}_{3} \mathrm{CCH}$ lines for a source size of $5^{\prime \prime}$. Least squares fits with and without opacity corrections are shown in red and blue, respectively. Lower panel: Same as the upper panel, but assuming a source size of $10^{\prime \prime} \ldots \ldots \ldots \ldots \ldots$

3.13 rotational diagram of the $\mathrm{CH}_{3} \mathrm{CCH}$ lines for a source size of $10^{\prime \prime}$. The points were divided into two subsets indicated by the dashed line. The points pertaining to the low- and high-energy subsets are shown in blue and red, respectively. Least squares fits to the points within each subset are shown. Circles and squares correspond to the lines with and without opacity cor-

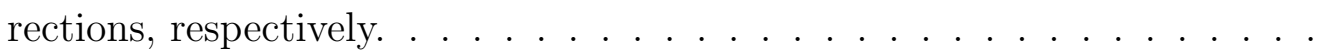

3.14 Peak velocities versus line frequency obtained from gaussian fittings to the $\mathrm{CH}_{3} \mathrm{CCH}$ lines. Each plot contains only transitions with the same K quantum number. . . . . . . . . . . . . . . . .

3.15 Line widths vs. rest frequencies obtained from gaussian fittings of the observed transitions. Each subplot contains only transitions with the same $\mathrm{K}$

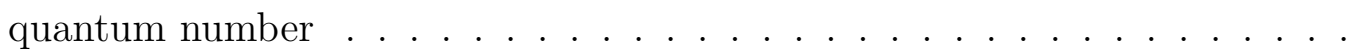

3.16 Line widths vs. peak velocities obtained from gaussian fittings of the observed transitions. Blue cross marks represent transitions with $\mathrm{K}=0$, purple squares represent transitions with $\mathrm{K}=1$, green circles represent transitions with $\mathrm{K}=2$ and red triangles represent transitions with $\mathrm{K}=3$. The black dashed line separates the blue- and red-shifted regions of the plot. G331 has a systemic velocity of $-90 \mathrm{~km} \mathrm{~s}^{-1}$.

3.17 Top pannel: Spectrum of G331 showing the $10_{0}-9_{0}$ and $10_{1}-9_{1}$ transitions of $\mathrm{CH}_{3} \mathrm{CCH}$ in solid red line. Dashed green lines are the Gaussian profile fittings. The red dash-dot line indicates the peak velocity of the $10_{0}-9_{0}$ transition. Bottom pannel: Same as the top pannel, but for the $18_{0}-17_{0}$ and $18_{1}-17_{1}$ transitions - in blue instead of red. . . . . . . . . . . . . 
A.1 Spectral bands: 170630-171030 MHz; 187690-188130 MHz; 204750$205220 \mathrm{MHz} \ldots \ldots \ldots \ldots \ldots \ldots$

A.2 Spectral bands: 221800-222320 MHz; 238840-239430 MHz; 255920$256520 \mathrm{MHz} \ldots \ldots \ldots \ldots 113$

A.3 Spectral bands: 272970-273610 MHz; 290030-290710 MHz; 307080$307800 \mathrm{MHz} \ldots \ldots \ldots \ldots \ldots$. . . . . . . . . . . . . . . . . . . . . . . . 



\section{List of Tables}

1.1 Generic gas-phase reactions and typical rate coefficients (Tielens, 2005, van Dishoeck, 2014). ${ }^{a}$ Highly reaction specific. ${ }^{b}$ In the exothermic direction.

${ }^{c}$ In the unshielded radiation field. ${ }^{d}$ In the exothermic direction and assuming no activation barrier. The rate coefficient units will depend on the rate expression and therefore on the order of the reaction. First-order and second-order reactions yield, respectively, $\mathrm{k}=\left[\mathrm{s}^{-1}\right]$ and $\mathrm{k}=\left[\mathrm{cm}^{3} \mathrm{~s}^{-1}\right] \ldots \ldots$

1.2 Elemental abundance with respect to total hydrogen in dark clouds. From the AL model in Acharyya and Herbst $(2017) \ldots \ldots . . \ldots$. . . . . . 28

2.1 Frequency channels of the SHeFI instrument. SIS stands for superconductorinsulator-superconductor mixer; SSB stands for single sideband receiver; HEB stands for hot electron bolometer mixer; and DSB stands for double sideband receiver. . . . . . . . . . . ...... 55

2.2 Frequency channels of the SEPIA instrument. SIS stands for superconductorinsulator-superconductor mixer and 2SB stands for dual sideband receiver.

3.1 Spectral lines of $\mathrm{CH}_{3} \mathrm{CCH}$ observed toward G331 and their parameters obtained from Gaussian fits.

3.2 Values of $\mathrm{T}_{e x c}$ and $\mathrm{N}_{\text {tot }}$ of $\mathrm{CH}_{3} \mathrm{CCH}$ toward $\mathrm{G} 331$ obtained from the analysis of the rotational diagrams. . . . . . . . . . . . . . . 68

3.3 Abundances of $\mathrm{CH}_{3} \mathrm{CCH}$ observed toward high- and low-mass star-forming sites. ${ }^{a}$ From Taniguchi et al. $(2019)$. . . . . . . . . . . . . . . 
3.4 Spectroscopic parameters employed in the spectrum simulations. The experimental measurements of the B rotational constant and the centrifugal distortion constants were obtained from Dubrulle et al. (1978). The dipole moment was measured by Muenter and Laurie (1966). Since the A rotational constant cannot be determined directly from the measured spectra, the estimated constant from the JPL database was used. . . . . . . . 73

3.5 Values of $\chi_{\text {red }}^{2}$ obtained from the least square fits of the traditional and modified rotational diagrams. . . . . . . . . . . . . . . . 79

3.6 Values of $\mathrm{T}_{\text {exc }}$ and $\mathrm{N}_{\text {tot }}$ of $\mathrm{CH}_{3} \mathrm{CCH}$ obtained from the 2-component rotational diagrams for a source size of $10^{\prime \prime} \ldots \ldots \ldots$. . . . . . . . . . 81 


\section{Contents}

1. Introduction . . . . . . . . . . . . . . . . 23

1.1 Interstellar chemistry . . . . . . . . . . . . . . . . . 24

1.1.1 Gas-phase processes . . . . . . . . . . . . . 24

1.1.1.1 Bond-formation processes ............ 25

1.1.1.2 Bond-destruction processes ........... 26 26

1.1.1.3 Bond-rearrangement processes . . . . . . . . . . 27

1.1.2 Solid-phase processes . . . . . . . . . . . . . . . 28

1.2 Massive star formation . . . . . . . . . . . . . . . . . . . . . . . . . . . . 29

1.2.1 Core-accretion model . . . . . . . . . . . . . . 32

1.2.2 Competitive-accretion model . . . . . . . . . . . 33

1.2.3 Protostellar-collision model . . . . . . . . . . . . . . . 33

1.3 Hot Molecular Cores . . . . . . . . . . . . . . . . . . . . . . . . 34

1.3.1 The HMC G331.512-0.103 . . . . . . . . . . . . 36

1.4 Spectral lines' fundamentals . . . . . . . . . . . . . . . . . . . 39

1.5 Rotational transitions . . . . . . . . . . . . . . . . . . 42

1.5.1 The types of rotors . . . . . . . . . . . . 43

1.5.2 The rotational Hamiltonian . . . . . . . . . . . 45

1.5.3 Dipole moment and selection rules . . . . . . . . . 46

1.6 Rotational diagrams . . . . . . . . . . . . . . . . 46

1.6.1 Opacity correction factor . . . ............. 48

1.6.2 Beam dilution factor . . . . . . . . . . . . . 49

1.7 The spectral tracer methyl acetylene . . . . . . . . . . . . . . . . . 49 
2. Facilities and methods . . . . . . . . . . . . . . 53

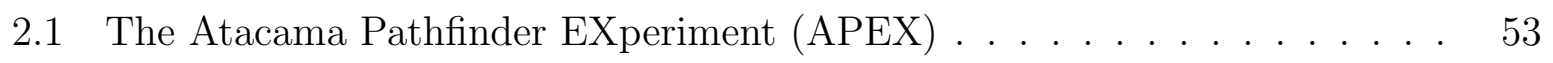

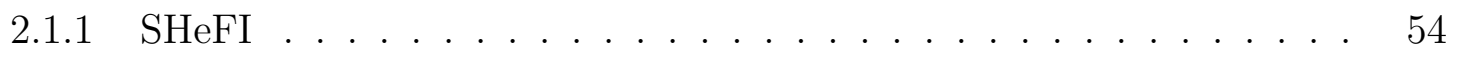

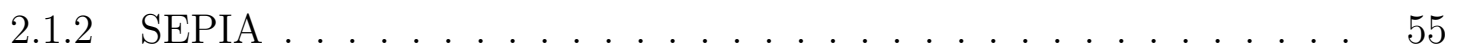

2.2 Observations and data calibration . . . . . . . . . 56

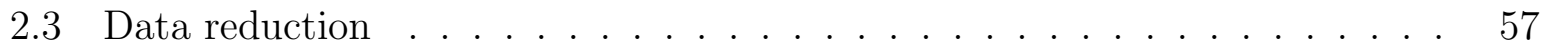

2.4 Line identification and gaussian fitting $\ldots \ldots \ldots \ldots \ldots$

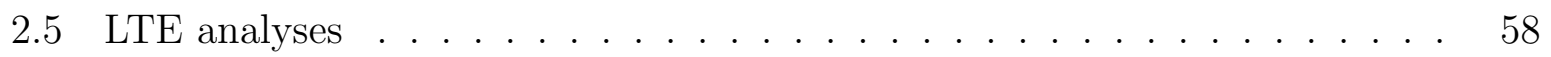

2.5 .1 Rotational diagrams . . . . . . . . . . . . . . 58

2.5.2 Synthetic LTE spectra . . . . . . . . . . . . . . . . 60

2.6 Rotational-spectrum simulations . . . . . . . . . . . . . . . . 61

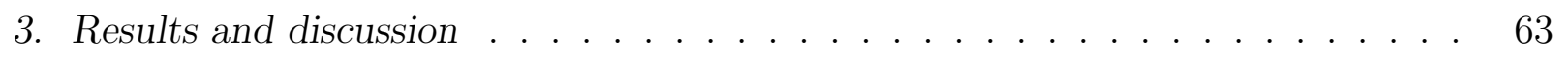

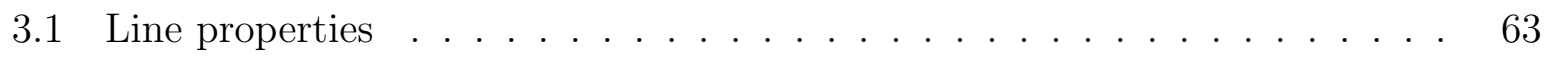

3.2 LTE analyses . . . . . . . . . . . . . . . . . . . . . 66

3.2 .1 Rotational diagrams . . . . . . . . . . . . . . . . 66 66

3.2 .2 Synthetic spectra . . . . . . . . . . . . . . 69

$3.2 .3 \mathrm{CH}_{3} \mathrm{CCH}$ abundance $\ldots \ldots \ldots \ldots \ldots \ldots$

3.3 Intensity inversion $\ldots \ldots \ldots \ldots \ldots \ldots \ldots$

3.3.1 Rotational-spectrum simulations . . . . . . . . . . . . . . 73

3.3.2 Inversion in the observed spectra $\ldots \ldots \ldots \ldots$. . . . . . 76

3.3.3 Modified rotational diagrams . . . . . . . . . . . . . . 78

3.3.4 Quantifying the temperature gradient . . . . . . . . . . 8 80

3.4 Kinematics . . . . . . . . . . . . . . . . . . . . . 82

3.4 .1 Velocity vs. frequency $\ldots \ldots \ldots$. . . . . . . . . . . 82

3.4 .2 FWHM vs. frequency . . . . . . . . . . . . . . . . . 82

3.4 .3 FWHM vs. velocity . . . . . . . . . . . . . . 885

4. Conclusions and future perspectives . . . . . . . . . . . . . . . 89

4.1 Conclusions . . . . . . . . . . . . . . . . . . . . . . 889

4.2 Future perspectives . . . . . . . . . . . . . . . . 91

Bibliography . . . . . . . . . . . . . . . . . 93 
A. Total spectrum of G331 . . . . . . . . . . . . . . . . . . . . 111

A.1 Here we present the total spectrum of G331, including the observations conducted with the SEPIA180, APEX-1 and APEX-2 receivers of the APEX $12 \mathrm{~m}$ telescope. . . . . . . . . . . . . . . . . 111 

Chapter 1

\section{Introduction}

Since the beginning of written history, humanity has contemplated the question as to what is the origin of the stars, planets, and ultimately life. The Greek philosopher Thales of Miletus is believed to be the first individual in Western civilization to explore the formation of the universe from a naturalistic standpoint, earning him the sobriquet "the first philosopher". According to his ideas, likely influenced by Mesopotamian beliefs, water would be the Primary Principle from which the cosmos and everything it encompasses come into being. Other presocratric thinkers, such as Leucippus and Democritus, also theorized on the elements that compose the natural world. They are regarded as the creators of the Atomist Doctrine, in which the world would be composed by two fundamentally different elements: atoms and void. The former are perfectly solid and indivisible bodies, which can only move about in the latter.

Later, during what the French philosopher Auguste Comte described as the third stage of human society, the combination of rational thinking and observation resulted in more solid theories to describe the origin of the stellar bodies. His epistemological arguments were preceded by the ideas of notable characters such as Galileo Galilei, Isaac Newton, Francis Bacon and René Descartes, who heavily contributed to the consolidation of the scientific method. Now, over 200 years after the beginning of Comte's so-called "positive stage", modern science has developed an impressive amount of facilities and techniques to explore thoroughly those fundamental questions. In this context, this master's thesis aims to contribute ever so slightly to our understanding of massive star formation processes, and its entanglement with the chemistry of the Interstellar Medium (ISM). 


\subsection{Interstellar chemistry}

To date, nearly 200 individual molecules, comprising 16 different elements, have been definitively detected in the interstellar and circumstellar media ${ }^{1}$. Those species, which are majorly organic, have also had numerous isotopologue detections. The chemical processes that occur in those environments and result in the formation of molecular species can be divided into two broad classes: reactions that take place on the surface of interstellar grains and reactions that occur in the gas phase. In the following sections, we provide a brief overview of those processes.

\subsubsection{Gas-phase processes}

Gas-phase reactions can be further separated into three different categories: bondformation processes, bond-destruction processes, and bond-rearrangement processes. In Table 1.1, we summarize the generic examples of those processes and their typical reaction rate coefficients.

Table 1.1 - Generic gas-phase reactions and typical rate coefficients (Tielens, 2005, van Dishoeck, 2014). ${ }^{a}$ Highly reaction specific. ${ }^{b}$ In the exothermic direction. ${ }^{c}$ In the unshielded radiation field. ${ }^{d}$ In the exothermic direction and assuming no activation barrier. The rate coefficient units will depend on the rate expression and therefore on the order of the reaction. First-order and second-order reactions yield, respectively, $\mathrm{k}=\left[\mathrm{s}^{-1}\right]$ and $\mathrm{k}=\left[\mathrm{cm}^{3} \mathrm{~s}^{-1}\right]$.

\begin{tabular}{lcc}
\hline \hline & \multicolumn{1}{c}{ Reaction } & Rate coefficient \\
\hline Bond-formation processes & & \\
Radiative association & $\mathrm{X}+\mathrm{Y} \rightarrow \mathrm{XY}+h \nu$ & $10^{-17}$ to $10^{-14} \mathrm{~cm}^{3} \mathrm{~s}^{-1}$ a \\
Associative detachment & $\mathrm{X}^{-}+\mathrm{Y} \rightarrow \mathrm{XY}+\mathrm{e}$ & $\sim 10^{-9} \mathrm{~cm}^{3} \mathrm{~s}^{-1} b$
\end{tabular}

\section{Bond-destruction processes}

$\begin{array}{llc}\text { Photodissociation } & \mathrm{XY}+h \nu \rightarrow \mathrm{X}+\mathrm{Y} & 10^{-10} \text { to } 10^{-8} \mathrm{~s}^{-1} c \\ \text { Dissociative recombination } & \mathrm{XY}^{+}+\mathrm{e} \rightarrow \mathrm{X}+\mathrm{Y} & 10^{-7} \text { to } 10^{-6} \mathrm{~cm}^{3} \mathrm{~s}^{-1}\end{array}$

\section{Bond-rearrangement processes}

\begin{tabular}{lcc} 
Ion-molecule exchange & $\mathrm{X}^{+}+\mathrm{YZ} \rightarrow \mathrm{XY}^{+}+\mathrm{Z}$ & $10^{-9}$ to $10^{-8} \mathrm{~cm}^{3} \mathrm{~s}^{-1} b$ \\
Charge-transfer & $\mathrm{X}^{+}+\mathrm{YZ} \rightarrow \mathrm{X}+\mathrm{YZ}^{+}$ & $10^{-9} \mathrm{~cm}^{3} \mathrm{~s}^{-1} b$ \\
Neutral-neutral & $\mathrm{X}+\mathrm{YZ} \rightarrow \mathrm{XY}+\mathrm{Z}$ & $10^{-11}$ to $10^{-9} \mathrm{~cm}^{3} \mathrm{~s}^{-1} d$ \\
\hline \hline
\end{tabular}

For unimolecular reactions, the rates of formation or destruction of a species are given

\footnotetext{
${ }^{1}$ see https://cdms.astro.uni-koeln.de/
} 
by:

$$
\frac{d n(A)}{d t}=-k n(A)=-\frac{d n(C)}{d t}
$$

Where $k$ is the reaction rate coefficient in $\mathrm{s}^{-1}$ and $n$ is the concentration in $\mathrm{cm}^{-3}$. Analogously, for bimolecular reactions:

$$
\frac{d n(A)}{d t}=-k n(A) n(B)=-\frac{d n(C)}{d t}
$$

Where $k$ is the reaction rate coefficient in $\mathrm{cm}^{3} \mathrm{~s}^{-1}$. Chemical processes that involve three or more bodies are only significant for densities above $10^{13} \mathrm{~cm}^{-3}$ (van Dishoeck, 2014), which is far too high for the regions explored in this work. In the following subsections, we give a panoptic view of the main chemical processes that occur in the gas-phase of the ISM.

\subsubsection{Bond-formation processes}

In radiative association reactions, the binding energy of the newly formed molecule is carried away through the emission of radiation, which stabilizes the collision product. With the exception of large molecules (e.g., Polycyclic Aromatic Hydrocarbons (PAHs)) or tightly bound species, those processes are typically very slow, and therefore are highly inefficient (van Dishoeck, 2014).

In a scenario where the reagent species form a long-lived activated complex before the emission of a photon and stabilization of the final product, the radiative-association process becomes more likely to happen. In this case, the reaction can be represented by:

$$
X+Y \underset{k_{d}}{\stackrel{k_{a}}{\rightleftharpoons}} X Y^{*} \stackrel{k_{r}}{\longrightarrow} X Y
$$

where $k_{a}, k_{d}$, and $k_{r}$ are the association, dissociation, and radiative stabilization rates, respectively. $X$ and $Y$ are the reactants and $X Y^{*}$ is the activated complex. This complex temporarily stores the kinetic energy and interaction energy as vibrational energy, which is eventually released through a vibrational transition accompanied by the emission of a photon. The radiative rate of those transitions is $\sim 10^{-3} \mathrm{~s}^{-1}$, which is usually much slower that collision timescales. For this reason, laboratory measurements of those rate coefficients are generally not feasible, and estimations are obtained through theoretical calculations. 
In associative-detachment reactions, the neutral product of the collision of an anion and an atom is stabilized by the emission of an electron. In general, reactions involving anions as reactants do not contribute significantly to the chemistry of the ISM (the formation of negative ions being the limiting step), although there is one notable exception. The reaction

$$
H+H^{-} \rightarrow H_{2}+e
$$

has a rate coefficient of $k=1.3 \times 10^{-9} \mathrm{~cm}^{3} \mathrm{~s}^{-1}$, and potentially played an important role in the formation of $\mathrm{H}_{2}$ in the early Universe (Tielens, 2005).

\subsubsection{Bond-destruction processes}

The UV radiation that permeates diffuse interstellar clouds leads to the destruction of molecular bonds through photodissociation (Millar and Williams, 1988). While typical bonding energies of molecules are below $10 \mathrm{eV}$, direct absorption into the dissociating continuum state induced by UV radiation is generally not an important effect. Instead, photodissociation more commonly occurs following one of the three routes: through a transition to the continuum of an electronically excited state, through the mixture of the excited electronic state with a dissociative state, or through the radiative decay of an excited electronic state to the continuum of the ground electronic state. In diffuse clouds, where there is an intense UV radiation flux, typical molecular lifetimes are of only 1001000 years van Dishoeck, 2014).

Upon penetrating interstellar clouds, the UV radiation is attenuated due to dust absorption and scattering effects. For this reason, the radiation field will depend on the opacity into the cloud. UV absorption by abundant species can also impact the penetration capability of the radiation and therefore the photodissociation rates. Those reactions are induced by absorption lines, which can in turn become optically thick. In this scenario, the molecular species lying deeper into the cloud are shielded from the radiation by the so-called "self-shielding" effect (van Dishoeck and Black, 1988). This phenomenon is particularly important for species such as $\mathrm{H}_{2}$ (Abgrall et al., 2000), $\mathrm{CO}$ (Visser et al., 2009), and $\mathrm{N}_{2}$ (Li et al., 2013; Heays et al., 2014).

Besides the external radiation field, UV photons can also be produced inside the cloud. Primary electrons generated by cosmic rays can electronically excite $\mathrm{H}_{2}$ molecules, which 
decay radiatively and release UV photons. Since cosmic rays are energetic enough to penetrate deep into the clouds, this mechanism result in a constant UV flux even at long depths.

Another bond-destruction process, dissociative recombination, involves the capture of an electron by a molecular ion, which forms a neutral species in an excited electronic state that can eventually dissociate. This process is usually very quick at low temperatures, thus efficiently destroying molecular ions.

\subsubsection{Bond-rearrangement processes}

Besides forming and destructing molecular bonds, chemical reactions can also lead to the formation of more complex species by means of bond-rearrangement processes. In the case of exothermic ion-molecule exchange, energy barriers can be easily overcome by their strong polarization-induced interaction potential, thus resulting in generally high rate coefficients. That is to say, ion-molecule reactions are usually very fast, and because of that they play an important role in the chemistry of the ISM. In general, those processes are driven either by cosmic ray ionization or photoionization, and their rate coefficients are independent of temperature. Reactions involving ions and molecules with permanent electric dipole moments (e.g., $\mathrm{C}^{+}+\mathrm{H}_{2} \mathrm{O} \rightarrow \mathrm{H}+\mathrm{HOC}^{+}$) have even larger rate coefficients, due to their enhanced long-range attraction. Furthermore, charge exchange reactions involving atoms are particularly important to the ionization balance of the environment, as is the case for $\mathrm{O}$ and $\mathrm{H}^{+}$in HII regions (Tielens, 2005).

Contrarily to ion-molecule reactions, neutral-neutral reactions usually involve considerable activation barriers, as a consequence of the bond-breaking associated with the molecular rearrangement. Since their attractive interaction due to van-der-Waals forces is weak, activation barriers can significantly hinder those processes. For this reason, neutralneutral reactions are generally important only for warm gas - such as in stellar ejecta or hot cores (Tielens, 2005).

Naturally, the importance of each reaction route in forming a particular species will depend on the elemental abundances, the enthalpy of the reaction (i.e., if it's endothermic or exothermic), and whether or not there is an activation barrier. Thus, exothermic reactions involving $\mathrm{H}$ and $\mathrm{H}_{2}$ usually dominate interstellar chemical networks. In Table 1.2 , typical low-metal elemental abundances used for modeling the chemistry of dark clouds are listed. 
Table 1.2 - Elemental abundance with respect to total hydrogen in dark clouds. From the AL model in Acharyya and Herbst (2017).

\begin{tabular}{cccc}
\hline Element & Abundance & Element & Abundance \\
\hline $\mathrm{H}_{2}$ & 0.5 & $\mathrm{~S}^{+}$ & $8 \times 10^{-8}$ \\
$\mathrm{He}$ & 0.09 & $\mathrm{Fe}^{+}$ & $3 \times 10^{-9}$ \\
$\mathrm{C}^{+}$ & $7.3 \times 10^{-5}$ & $\mathrm{Na}^{+}$ & $2 \times 10^{-9}$ \\
$\mathrm{~N}$ & $2.14 \times 10^{-5}$ & $\mathrm{Mg}^{+}$ & $7 \times 10^{-9}$ \\
$\mathrm{O}$ & $1.76 \times 10^{-4}$ & $\mathrm{Cl}^{+}$ & $1 \times 10^{-7}$ \\
$\mathrm{~F}$ & $1.8 \times 10^{-8}$ & $\mathrm{P}^{+}$ & $2 \times 10^{-10}$ \\
$\mathrm{Si}^{+}$ & $8 \times 10^{-9}$ & & \\
\hline \hline
\end{tabular}

\subsubsection{Solid-phase processes}

The interstellar solid-phase chemistry happens in the surface of dust particles that are shrouded by ice mantles. Those bodies have a layered structure consisting of a carbonaceous core, followed by a layer of water-rich ice and a layer of water-poor ice (see Figure 1.1). This stratified shape is due to the condensation of the ice: at the earlier evolutionary stages of the cloud, hydrogenation of atomic oxygen forms the water-rich layer; then, the freeze-out of CO leads to the formation of the CO-rich and water-poor layer (van Dishoeck, 2014). Those icy surfaces act as catalysts (Tielens, 2005), facilitating the formation of many different molecular species even without any energy input (e.g., see Linnartz et al. 2015; Qasim et al. 2020).

The three major mechanisms whereby grain-surface chemistry occurs are the LangmuirHinshelwood, the Eley-Rideal, and the Harris-Kasemo - also known as hot atom. In the first one, also called the diffusive mechanism, the accreted reactants first reach thermal equilibrium with the surface, and then one or both diffuse through the biding sites of the ice until reaching one-another and reacting. In the Eley-Rideal mechanism, the gas-phase species does not diffuse, but rather lands on top of another species and promptly reacts, without reaching thermal equilibrium beforehand. Finally, in the hot-atom mechanism, the accreted reactant diffuses through the ice and reacts with another adsorbed species before reaching thermal equilibrium (Herbst and van Dishoeck, 2009; Linnartz et al., 2015). In Figure 1.2, we present a schematic view of these three processes.

While grain-surface reactions indeed contribute significantly to the chemical inventory of the ISM, solid-phase species lack rotational degrees of freedom and therefore are not 
a) Early ice formation

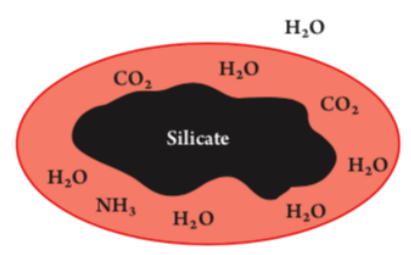

b) Cloud core ice formation

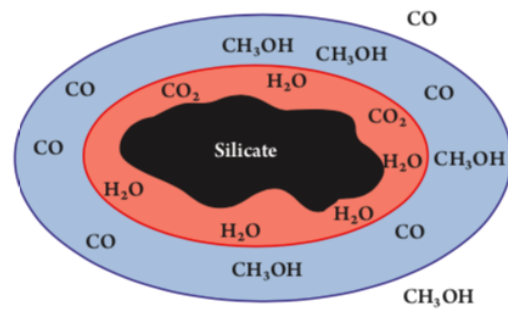

c) Cold (<20 K) UV.processing

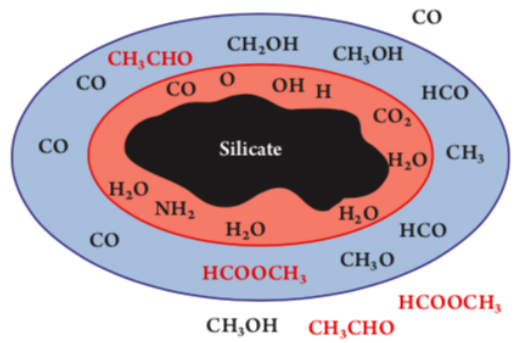

d) Lukewarm protostellar envelope $>20 \mathrm{~K}$

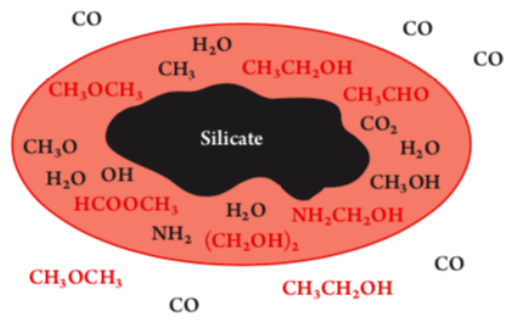

e) Protostellar hot core $>100 \mathrm{~K}$

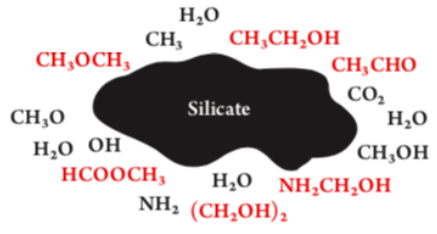

Figure 1.1: From van Dishoeck (2014). Formation and evolution of the interstellar ices. The $\mathrm{H}_{2} \mathrm{O}-$ rich ice is shown in red, while the CO-rich ice is shown in blue.

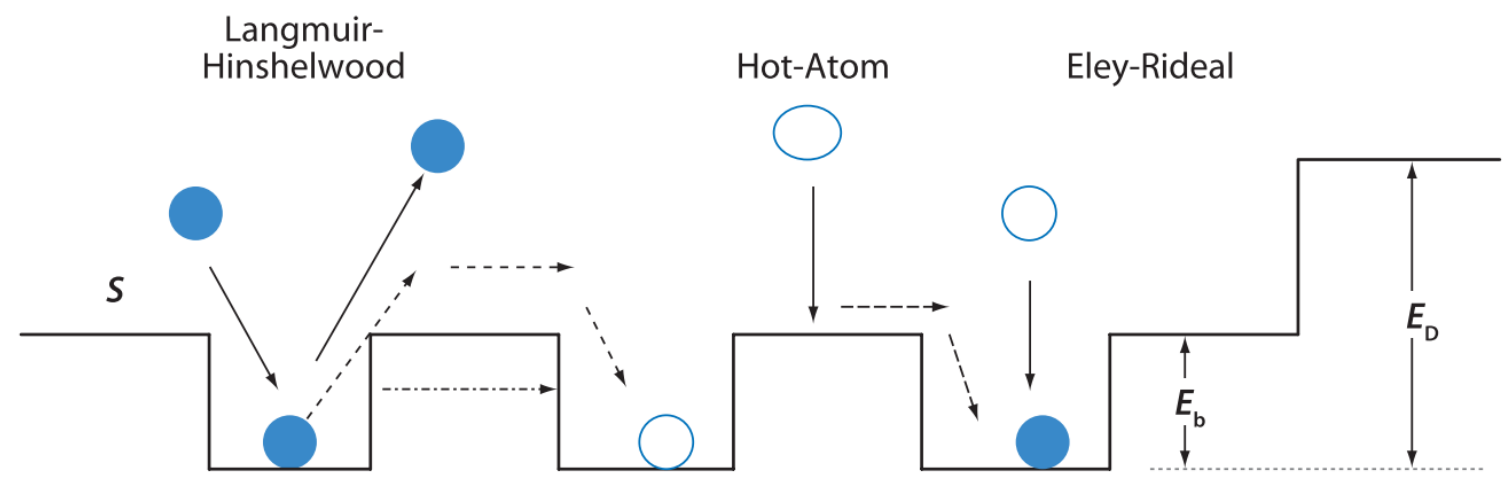

Figure 1.2: Sketch of the three major surface reaction mechanisms. From Herbst and van Dishoeck (2009).

typically observable with radio telescopes. For this reason, the present work focuses on gasphase processes. The reader is referred to specialized literature (e.g., Williams and CecchiPestellini 2016 and the references cited in this subsection) for more in-depth discussions on interstellar solid-phase chemistry.

\subsection{Massive star formation}

Massive stars, as the name suggests, are characterized by gargantuan masses $(M>$ $\left.8 M_{\odot}\right)$ and luminosities $\left(L>10^{3} L_{\odot}\right)$. Through turbulence-enhancing phenomena, such as massive outflows and expanding HII regions, they greatly affect the energy budget of 
the galaxies. Moreover, they are the main sources of heavy-element and UV-radiation injection into the ISM. Thus, massive stars play a crucial role in shaping galactic structure and evolution.

They are born within cloud corpuscles of $\sim 1-10$ pc and $\sim 10-10^{6} \mathrm{M}_{\odot}$, known as massive clumps, which in turn are predominantly observed in hyper-massive and hyper-dense molecular clouds (Tan, 2018). The formation process of those stars, however, is still poorly understood. While, for low-mass stars, there is a solid and well-established evolutionary sequence (see Shu et al. 1987; Andre et al. 2000), many open questions still remain for the massive counterparts. The difficulty associated with the study of massive star formation is mostly due to their substantially shorter evolutionary timescale $\left(t_{K H} \leq 10^{4}\right.$ yr for O-type stars), combined with observational constrains, such as their complex cluster environments $\left(n_{\star} \gtrsim 100 p c^{-3}\right)$ and large distances involved $(d \geq 1 k p c)$ Garay and Lizano, 1999; Zinnecker and Yorke, 2007; Silva et al., 2017). Inasmuch as the observational study of those environments continues to be hampered by their scarcity and other intrinsic limitations, theoretical models that incorporate astrochemistry are key to successfully work toward building a massive star formation paradigm (see Tan 2018).

Massive gas clumps are defined following Tan et al. (2014) and Tan (2018), in which they are self-gravitating structures that fragment into massive star clusters. According to the core-accretion model of massive star formation (see \$1.2.1), this fragmentation occurs through the formation of dense, self-gravitating cores, which in turn collapse to form either single or multiple stars. A schematic view of the different levels of fragmentation involved in the formation of massive stars is shown in Figure 1.3, from Sadaghiani et al. (2020).

In the first hierarchical level, there is the example molecular cloud NGC 6334, which has an elongated filamentary structure. This filament is fragmented into clumps with masses of a few hundred $\mathrm{M}_{\odot}$. The clumps are in turn fragmented into cores, within which the stars are formed. Furthermore, observations and numerical models indicate that some of these cores can be further fragmented to form bound clusters, at scales between 500-5000 au (Offner et al., 2010; Hunter et al., 2014, Brogan et al., 2016; Sadaghiani et al., 2020). In the following subsections, we briefly outline the main formation scenarios of massive stars embedded in those structures. 


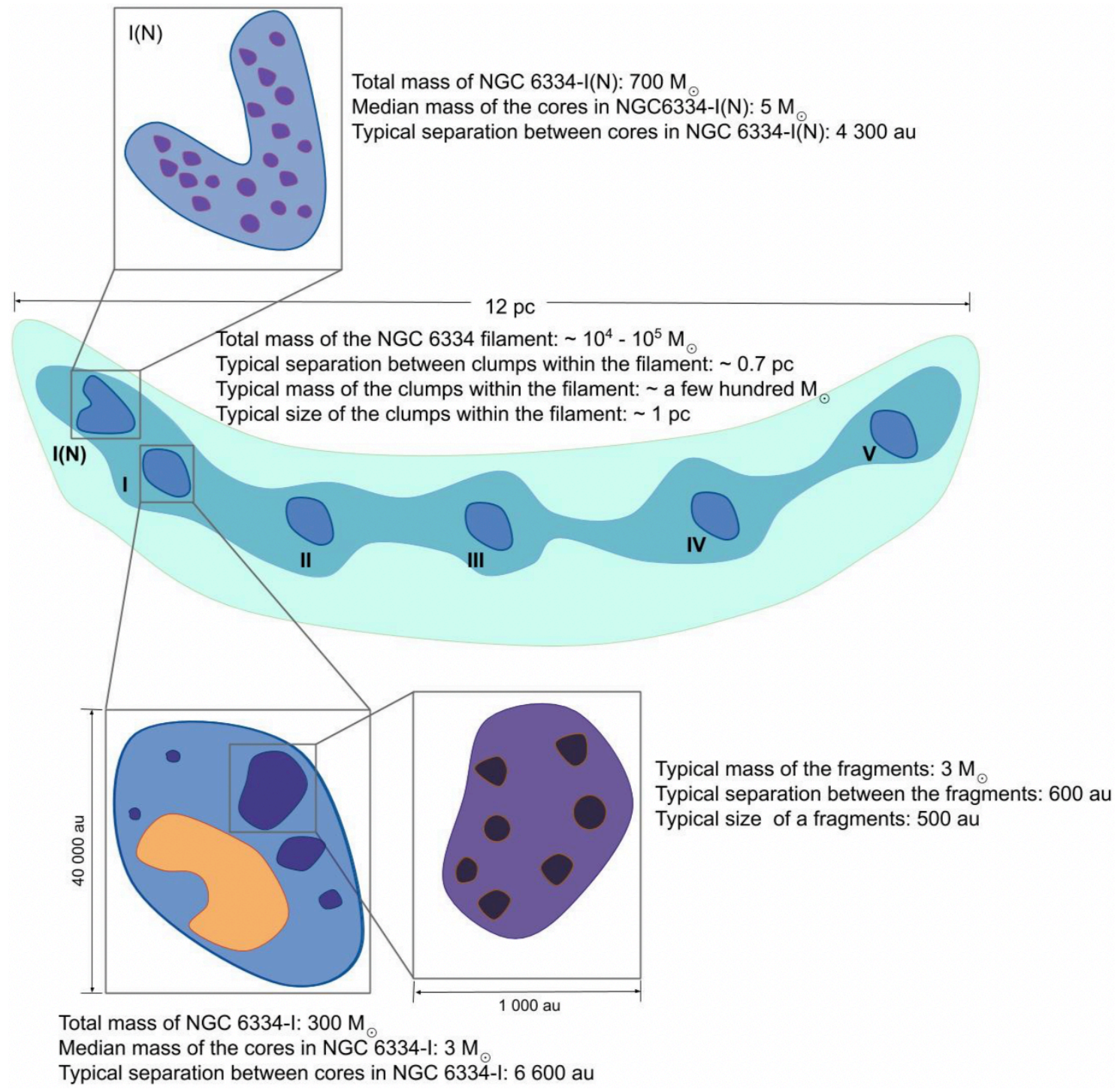

Figure 1.3: Figure from Sadaghiani et al. 2020), showing the different fragmentation levels of the NGC 6334 star-forming complex from the scale of the filament down to the scale of individual dense cores and protostars. The colors indicate different densities - lighter colors correspond to lower densities, whereas darker colors indicate higher densities - as well as ionized gas (in orange). Vertical and horizontal orientations correspond to the Galactic latitude and longitude coordinates, respectively. The distribution is based on observations by Russeil et al. (2013). 


\subsubsection{Core-accretion model}

The core-accretion model is the most conservative and simplest approach to massive star formation. It treats those processes as simply scaled-up versions of low-mass star formation theories, in which gas accretion occurs when gravity overcomes the thermal pressure gradient (Shu et al., 1987; Lada and Kylafis, 1999). In this theory, the final mass of the star is related to the mass of the core from which it forms. Thus, it predicts that the initial mass function is determined by the core mass function.

For a single, isothermal sphere, the low-mass accretion rate follows (Shu, 1977):

$$
\dot{m}_{*}=0.975 \frac{c_{t h}^{3}}{G}=4.36 \times 10^{-6}\left(\frac{T}{20 K}\right)^{3 / 2} M_{\odot} y r^{-1}
$$

where $c_{t h}^{3}$ corresponds to the isothermal sound speed and assuming $n_{H e}=0.2 n_{H_{2}}$. However, some difficulties arise when we expand this theory to high-mass stars. Since the predicted accretion rate is a function of only the gas temperature, and since they are positively correlated, the first massive stars that form in a region (that is, from a gas with T 10-20 K) will have low accretion rates and thus will take too long to form. To address this issue, McKee and Tan (2003) propose the so-called Turbulent Core Model (TCM), in which the internal supersonically turbulent motions observed in the cores induce internal substructures that then lead to an increase in the accretion rate with time (Myers and Fuller, 1992; Caselli and Myers, 1995; McLaughlin and Pudritz, 1997). This model is based on the fact that Equation 1.5 holds approximately when the non-thermal effects of turbulence and magnetic fields are incorporated (Stahler et al., 1980; Shu et al., 1987). Therefore, the pressure support of the massive cores is provided by turbulence and/or magnetic fields. As a result, the free-fall timescale of the dense cores will be shortened, and the accretion rates will be higher, once they become unstable and collapse.

One of the challenges of the core accretion model is related to the fact that the dense cores have many Jeans masses ${ }^{2}$ for a temperature of $\sim 10 \mathrm{~K}$ (Tan et al., 2014). Thus, instead of forming a single massive star, it should fragment further into forming a cluster of low-mass stars. This problem is addressed by McKee and Tan (2003), in which they assume that most of the density fluctuations in a core at a given moment are gravitationally

\footnotetext{
${ }^{2}$ The Jeans mass corresponds to the mass threshold above which the cloud undergoes gravitational collapse.
} 
stable. Furthermore, Krumholz (2006) suggested that the radiative feedback from the first stars would heat up the surrounding gas in the core and thus suppress the fragmentation. This was later confirmed by a number of simulations (see Krumholz and Bonnell 2007; Krumholz et al. 2010, 2011).

\subsubsection{Competitive-accretion model}

In the competitive-accretion model (Bonnell et al., 2001; Wang et al., 2010), the massive clumps fragment into protostellar seeds with initial masses of the order of the thermal Jeans mass. Then, they later accrete the surrounding gas, eventually forming massive stars. Unlike the core-accretion model, in this theory the gas is initially not gravitationally bound to the star. Instead, it is drawn chaotically from a wider region of the clump. In other words, the protostellar seeds are formed through gravitational collapse, but then grow by tidally-modified Bondi-Hoyle $]^{3}$ accretion, competing for the mass available in the clump.

The environment where those seeds are formed is highly dense and clustered. The number of stellar objects directly affects the mass accretion rates of the cluster members, thus signaling a causal relationship between the formation processes of clusters and massive stars. As a consequence of this model, massive stars should be segregated toward the center of the cluster, where the global clump infall leads to high gas densities.

This model also presents intrinsic difficulties that have yet to be solved. One of them is related to the radiation pressure feedback of the star, which disrupts the Bondi-Hoyle accretion for stellar masses above $\sim 10 \mathrm{M}_{\odot}$ (Edgar, 2004). As a result, the competitive accretion should not operate for stellar masses above this threshold. Moreover, this model also does not account for turbulent (virialized) media, in which the accretion rate would be too small to allow formations within timescales $\lesssim 1$ Myr (Krumholz et al., 2005).

\subsubsection{Protostellar-collision model}

In the protostellar-collision model, high-mass stars are proposed to be formed via direct collisions of lower-mass protostars (Bonnell et al., 1998; McKee and Tan, 2003). Because they are optically thick, merging protostars are not affected by stellar feedback, thereby

\footnotetext{
${ }^{3}$ Bondi-Hoyle accretion is a spherical accretion onto a compact object moving through a uniform gas free of self-gravity. For more information, see Edgar $(\overline{2004})$ and references therein.
} 
overcoming the problem of radiation pressure halting massive star formation. This model also yields high accretion rates - and, consequently, short formation timescales. However, in order to account for the stellar evolution timescale, those collisions should take place during a brief period in which the protostellar and stellar densities of a forming massive cluster must reach notably high values of $\sim 10^{8}$ stars pc $^{-3}$ (e.g., Bonnell et al. 1998; Bonnell and Bate 2002). Such a hypothesized ultradense region should presumably be very luminous, and yet has never been observed. Thus, this model is unlikely to play an important role in typical massive star-forming regions.

\subsection{Hot Molecular Cores}

Despite the lack of a thoroughly defined evolutionary sequence, some precursors of highmass stars are well established. One example are Hot Molecular Cores (HMCs), which are believed to be one of the first observable stages of massive star formation (Osorio et al. 1999). HMCs are characterized by compact sizes $(\lesssim 0.1$ pc), relatively high temperatures $(\gtrsim 100 \mathrm{~K})$ and high densities $\left(n_{H_{2}} \sim 10^{5}-10^{8} \mathrm{~cm}^{-3}\right)$, as well as large luminosities $\left(>10^{4}\right.$ $\left.\mathrm{L}_{\odot}\right)$ and masses $\left(\sim 10-1000 \mathrm{M}_{\odot}\right)$ (Heaton et al., 1989; Gomez et al., 1995; Cesaroni et al., 1998). They are associated with a rich molecular emission spectrum, including high-excitation transitions of multifarious "exotic" molecules (e.g., Comito et al. 2005), as well as $\mathrm{H}_{2} \mathrm{O}$ masers, and, oftentimes, ultracompact (UC) HII regions (Cesaroni, 2005). The HMC phase is thought to last from $\sim 10^{5}$ yr (van Dishoeck and Black, 1988) to $\sim 10^{6}$ yr (Garrod and Herbst, 2006). Comparatively, smaller regions ( $\lesssim 100 \mathrm{au})$ with similar characteristics are observed around solar-type protostars. Those low-mass counterparts are designated "hot-corinos" (Cazaux et al., 2003; Bottinelli et al., 2004; Ceccarelli, 2005).

HMCs are embedded in more extended molecular clumps, typically 10 times larger and more massive than the cores themselves (Cesaroni et al., 1991; Plume et al., 1997; Hofner et al., 2000). The temperature and density profiles of those clumps vary as:

$$
\begin{aligned}
& n \propto R^{-p} \\
& T \propto R^{-q}
\end{aligned}
$$

where $\mathrm{R}$ is the distance from the core. Generally, $p \simeq 1.5-2.5$ and $q \simeq 0.5$ (Hatchell et al., 2000; Fontani et al., 2002). In Figure 1.4, an image of a HMC observed toward IRAS 


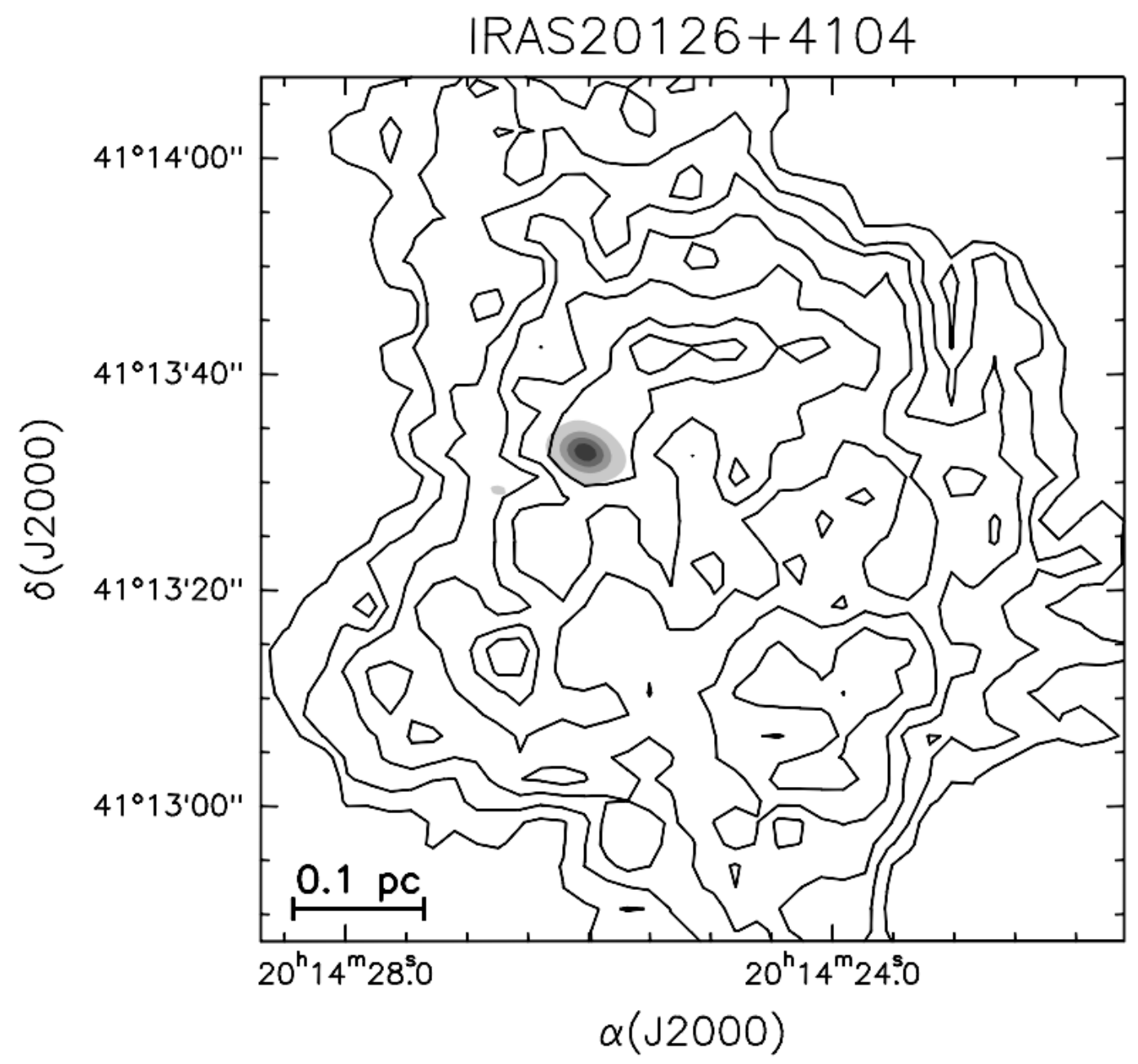

Figure 1.4: Superimposed maps of the $\mathrm{CH}_{3} \mathrm{CN}(5-4)$ traced $\mathrm{HMC}$ in the high-mass star-forming region IRAS $20126+4104$ (grey scale) and the $\mathrm{C}^{17} \mathrm{O}(2-1)$ traced surrounding molecular clump (contours). From Cesaroni 2005 .

$20126+4104$ in the $\mathrm{CH}_{3} \mathrm{CN}(5-4)$ line is shown in combination with a $\mathrm{C}^{17} \mathrm{O}(2-1)$ map of the surrounding molecular clump (Cesaroni, 2005).

The velocities associated with HMCs are virtually indistinguishable from those of the corresponding clumps. Moreover, Fontani et al. (2002) have shown that the density profile of the clump agrees with the mean density of the embedded HMC. Thus, the distinction between HMCs and their surrounding clump (i.e., the HMC diameter) can be blurry. Indeed, a more accurate description would be that HMCs correspond to a particular region inside the clump where density and temperature peak, as a result of the Massive Young Stellar Objects (MYSOs) embedded in them. This hypothesis is supported by the high masses and luminosities associated with HMCs, as well as the recurrent observations of $\mathrm{H}_{2} \mathrm{O}$ masers and UC HII regions toward those sources. Hence, HMCs could be further 
perceived as the very sites where collapse presumably takes place and fragmentation leads to star formation (see Cesaroni 2005).

The presence of powerful molecular outflows in many observed HMCs (e.g., Cesaroni et al. 1997, 1998; Gibb et al. 2004; Zhang et al. 2007; Bronfman et al. 2008; Qiu et al. 2008) also agrees with this scenario, in which it is speculated that the outflows determine the last stages of the HMC phase and the subsequent development of UC HII regions Cesaroni, 2005). Outflows provide optically thin channels through which the radiation can escape, thus reducing the radiation pressure barrier on the infalling gas and enabling the accretion onto the protostar (see, e.g., Kuiper and Yorke 2013). The resulting intense mass-accretion rates quench the development of an HII region around the massive star (Walmsley et al. 1995; Keto, 2002, 2003). The fact that free-free emission is not usually detected in those sources supports the hypothesis that HMCs precede UC HII regions.

\subsubsection{The HMC G331.512-0.103}

The radio source G331.5-0.1 is located at the tangent point of the Norma spiral arm, in the Southern Galaxy, at a distance of $\sim 7.5 \mathrm{kpc}($ Bronfman et al., 1989, 2008). It consists of an extended, exceptionally luminous, and massive region of ionized gas at the center of a Giant Molecular Cloud (GMC) with a FIR luminosity of $3.6 \times 10^{6} \mathrm{~L}_{\odot}$ and with a total CO mass of $8.7 \times 10^{6} \mathrm{M}_{\odot}($ Bronfman et al., 2008; Merello et al., 2013a). Hydrogen recombination lines yield a systemic velocity of $-89 \mathrm{~km} \mathrm{~s}^{-1}$ for this source Caswell and Haynes, 1987). Moreover, as evinced by its high luminosity and intense maser emission of $\mathrm{OH}$ and $\mathrm{CH}_{3} \mathrm{OH}$ (Nyman et al., 2001; Bronfman et al., 2008; Mendoza et al., 2018), as well as its various observed UC HII regions (Bronfman et al., 2000), this cloud harbors active sites of massive star formation.

The radial distribution of massive star forming regions in the Galactic disk is correlated with the azimuthally averaged radial distribution of the molecular gas - also known as the "molecular ring" - for all galactocentric radii. In the southern Galaxy, both molecular and massive-star-formation-rate rings peak within the range $0.5 \leq \mathrm{R} / \mathrm{R}_{\odot} \leq 0.6$ (Bronfman et al. 2000), in a region that coincides with the location of the parent GMC (García et al. 2014). Indeed, this GMC consists of one of the most massive and active star forming clouds in the southern Galaxy. Likewise, the Norma spiral arm seems to be one of the most active spiral features in forming massive stars. García et al. (2014) have derived a massive star 
formation rate per unit $\mathrm{H}_{2}$ mass $(\mathrm{MSFR})$ of $0.58 \pm 0.09 \mathrm{~L}_{\odot} / \mathrm{M}_{\odot}$ for the most massive clouds in the Norma arm. Comparatively, the average MSFR for their entire sample of GMCs was of $0.41 \pm 0.06 \mathrm{~L}_{\odot} / \mathrm{M}_{\odot}$. Conceivably, the MSFR of the most massive GMCs within the Norma arm, including G331.5-0.1, could even be used as a standard scale to compare the massive star formation activity in the rest of the Galaxy (as suggested by García et al. 2014).

Dust continuum observations at $1.2 \mathrm{~mm}$ have revealed six massive dust concentrations spread along the source, with a total mass of $\sim 1.4 \times 10^{4} \mathrm{M}_{\odot}($ Nyman et al., 2001). Within the brightest of them, Bronfman et al. (2008) observed a massive and powerful molecular outflow-namely, G331.512-0.103 - by means of line emission maps of the entire G331.50.1 core in the $\mathrm{CS}(7-6),{ }^{13} \mathrm{CO}(3-2), \mathrm{CO}(3-2), \mathrm{CO}(4-3)$, and $\mathrm{CO}(7-6)$ transitions. The molecular outflow/HMC G331.512-0.103 (henceforth G331) is one of the most distinctive HMCs known to date. It is associated with a very rich emission spectrum, including simple hydrocarbons, radical species, and a plethora of complex organic molecules (Merello et al., 2013a b; Mendoza et al., 2018; Duronea et al., 2019; Hervías-Caimapo et al., 2019). Observational evidence indicates that a MYSO at the center of G331 drives a powerful outflow with $\sim 55 \mathrm{M}_{\odot}$ of mass and a momentum of $\sim 2.4 \times 10^{3} \mathrm{M}_{\odot} \mathrm{km} \mathrm{s}^{-1}$, with lobes closely aligned with the line of sight. The shock of the outflow against the surrounding dense gas produces UV photons that, combined with the protostellar radiation, result in free-free emission that is observed in the radio continuum (Bronfman et al., 2008; Merello et al., 2013b). Moreover, observations with the Atacama Large Millimeter/submillimeter Array (ALMA) of the $\mathrm{SiO}(8-7), \mathrm{H}^{13} \mathrm{CO}^{+}(4-3), \mathrm{HCO}^{+}(4-3)$, and $\mathrm{CO}(3-2)$ transitions revealed an expanding shocked shell driven by stellar winds, which probably arises from the protostar confined in a hyper-compact HII region of $\sim 5 \operatorname{arcsec}$ (projected size of $\sim 0.2 \mathrm{pc}$ ). Those observations lead to an estimated source age of 2000 yr, making G331 one of the youngest hot cores/molecular outflows known to date (Merello et al., 2013b). In Figure 1.5, from Hervías-Caimapo et al. (2019), integrated emission maps of G331 in multiple transitions are shown, together with the $862 \mu \mathrm{m}$ continuum emission map.

As shown in Figure 1.5, Hervías-Caimapo et al. (2019) have conducted ALMA observations of various molecular transitions toward G331. They concluded that the observed molecular lines could be divided into two groups based on their widths: one that presents narrow lines that trace the core ambient gas (e.g., $\mathrm{CH}_{3} \mathrm{OH}, \mathrm{CH}_{3} \mathrm{CCH}, \mathrm{H}^{13} \mathrm{CO}^{+}$), and one 


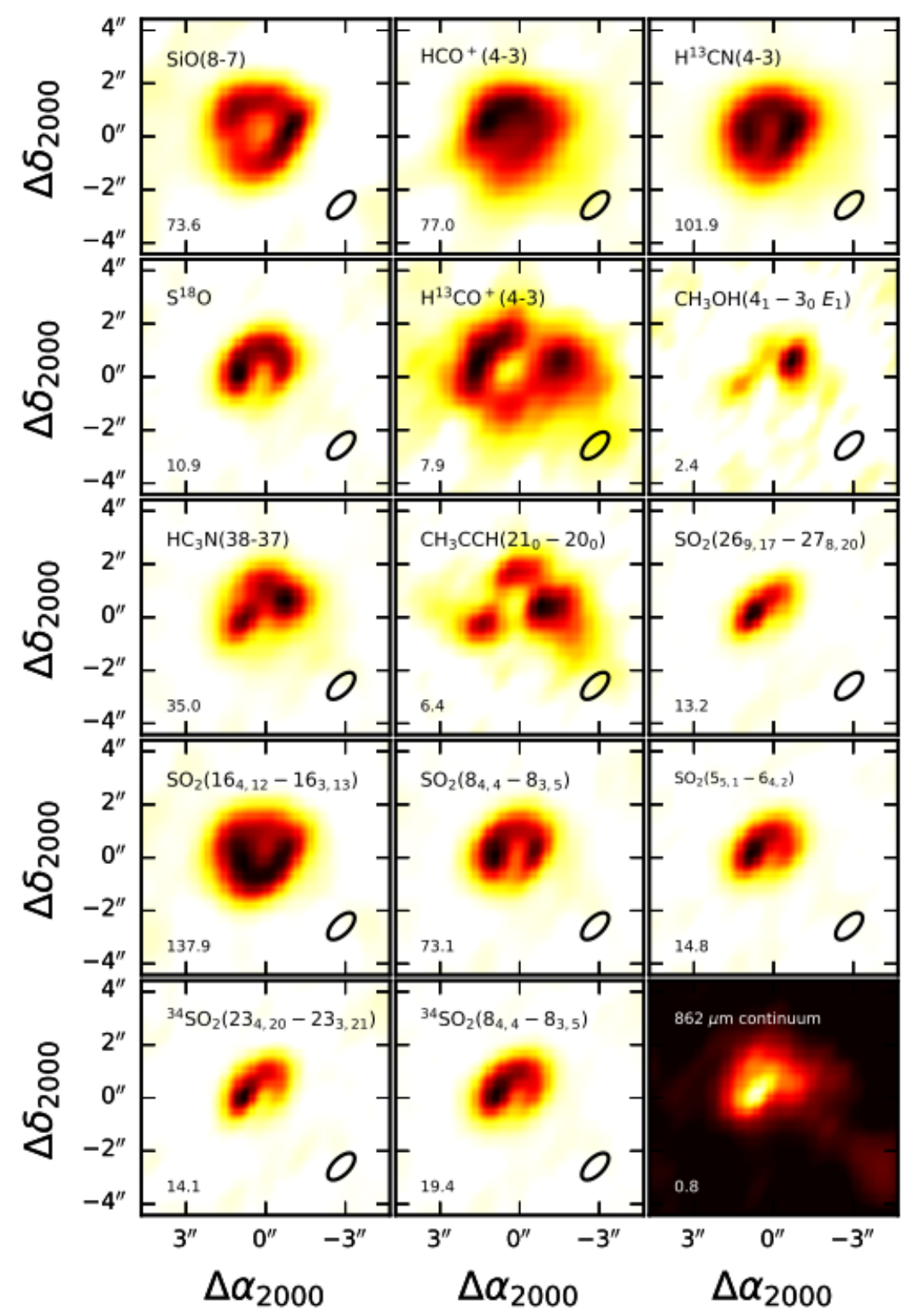

Figure 1.5: Zeroth moment maps of emission from G331, integrated in the range of the systemic velocity. The $862 \mu \mathrm{m}$ continuum emission map is also shown (bottom right panel). From Hervías-Caimapo et al. (2019). 


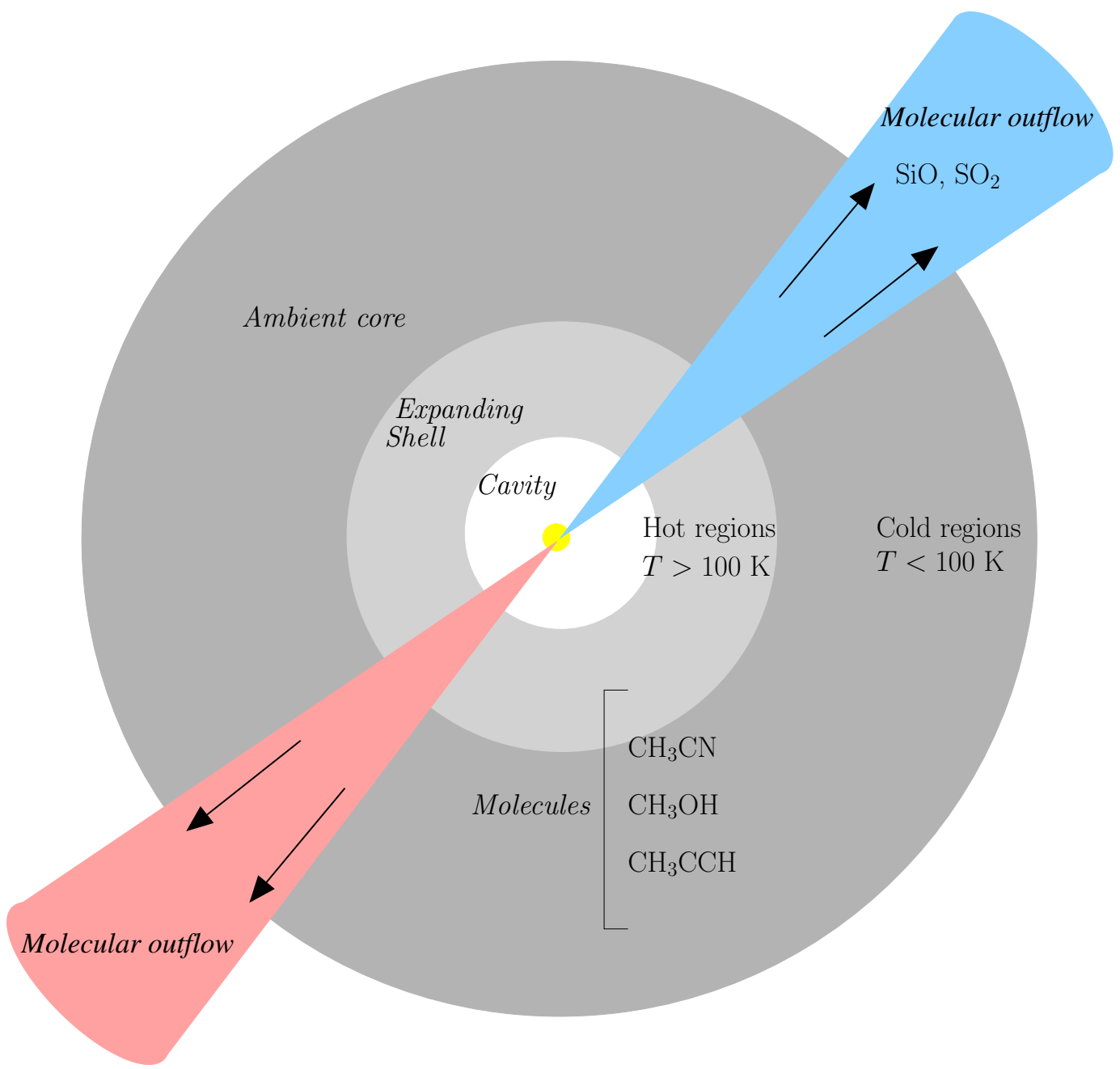

Figure 1.6: Schematic view of the physical model proposed for G331. Based on the models of Merello et al. (2013a); Duronea et al. (2019); Hervías-Caimapo et al. (2019)

that presents broader lines that trace the high-velocity outflow (e.g., $\mathrm{HC}_{3} \mathrm{~N}, \mathrm{SiO}, \mathrm{S}^{18} \mathrm{O}$, $\left.\mathrm{HCO}^{+}, \mathrm{H}^{13} \mathrm{CN}\right)$. Thus, different molecules will give information on different morphological features of this source. The ambient core is characterized by lower temperatures $\left(\mathrm{T}_{K} \sim 70 \mathrm{~K}\right)$, whereas the outflow yields higher values $\left(\mathrm{T}_{K} \sim 150-200 \mathrm{~K}\right)$. Duronea et al. (2019) further speculate that the $\mathrm{HC}_{3} \mathrm{~N}$ emission originates from an intermediate region between the ambient core and the expanding shell, based on the rotational temperatures they obtained. A schematic view of the physical model of G331 is presented in Figure 1.6.

\subsection{Spectral lines' fundamentals}

The interaction of a ray of electromagnetic radiation with matter can result in the addition or subtraction of energy from the ray by either emission or absorption. The 
energy emitted by the matter will result in an intensity addition to the beam Rybicki and Lightman, 1985):

$$
d I_{\nu}=j_{\nu} d s
$$

where $j_{\nu}$ is the monochromatic emission coefficient and $d s$ is the distance traveled by the beam. Conversely, the loss of intensity in a beam due to absorption by the matter is described by:

$$
d I_{\nu}=-\alpha_{\nu} I_{\nu} d s
$$

where $\alpha_{\nu}$ is the absorption coefficient, defined as $\alpha_{\nu}=n \sigma_{\nu}$, to wit: the product of the density $n$ of the absorbing particles times their absorption cross section $\sigma_{\nu}$. Since both "true" absorption and stimulated emission are proportional to the intensity of the incoming beam, both phenomena are considered as "absorption" in Equation 1.8 .

The combined effects of emission and absorption result in an expression for the variation of specific intensity along a ray (Rybicki and Lightman, 1985):

$$
\frac{d I_{\nu}}{d s}=-\alpha_{\nu} I_{\nu}+j_{\nu}
$$

Equation 1.9 is know as the Radiative Transfer Equation. In the limiting cases of either no absorption or no emission, its solutions are:

- Emission only: In this case, $\alpha_{\nu}=0$ and $\frac{d I_{\nu}}{d s}=j_{\nu}$. This expression has the solution:

$$
I_{\nu}(s)=I_{\nu}\left(s_{0}\right)+\int_{s_{0}}^{s} j_{\nu}\left(s^{\prime}\right) d s^{\prime}
$$

- Absorption only: In this case, $j_{\nu}=0$, and $\frac{d I_{\nu}}{d s}=-\alpha_{\nu} I_{\nu}$. This expression has the solution:

$$
I_{\nu}(s)=I_{\nu}\left(s_{0}\right)+\exp \left[-\int_{s_{0}}^{s} \alpha_{\nu}\left(s^{\prime}\right) d s^{\prime}\right]
$$

When the medium is under Thermodynamic Equilibrium, the radiation is in complete equilibrium with its surroundings, and the brightness distribution can be described by the Planck function: 


$$
\begin{gathered}
\frac{d I_{\nu}}{d s}=0 \\
I_{\nu}=\frac{j_{\nu}}{\alpha_{\nu}}=B_{\nu}(T)=\frac{2 h \nu^{3}}{c^{2}} \frac{1}{e^{h \nu / k T}-1}
\end{gathered}
$$

The concepts hitherto presented apply for both continuous and line radiation. However, since this thesis revolves around molecular lines, we will henceforth focus the discussion on the latter. The expression $j_{\nu}=\alpha_{\nu} B_{\nu}$ (Equation 1.13), also know as Kirchhoff's law, implies the existence of a relationship between emission and absorption at a microscopic level. This relationship is described by the Einstein coefficients, which represent the probability of a system to make a transition from one energy level to another either by absorbing or emitting a photon. The Einstein coefficients are associated with three different processes (Rybicki and Lightman, 1985):

- Spontaneous emission: This process occurs when the system drops from a level with higher energy to a level with lower energy, resulting in the emission of a photon. It occurs even in the absence of a radiation field. It is described by the Einstein A coefficient:

$$
A_{j i}=\text { transition probability per unit time for spontaneous emission }
$$

- Absorption: The system makes a transition from a lower-energy level to a higherenergy level by absorbing a photon. The probability for this process is proportional to the density of photons:

$$
B_{i j} \bar{J}=\text { transition probability per unit time for absorption }
$$

where the Einstein B coefficient is the proportionality constant.

- Stimulated emission: This process is also proportional to $\bar{J}$, but causes the emission of a photon. Thus, we have another Einstein B coefficient:

$B_{j i} \bar{J}=$ transition probability per unit time for stimulated emission 


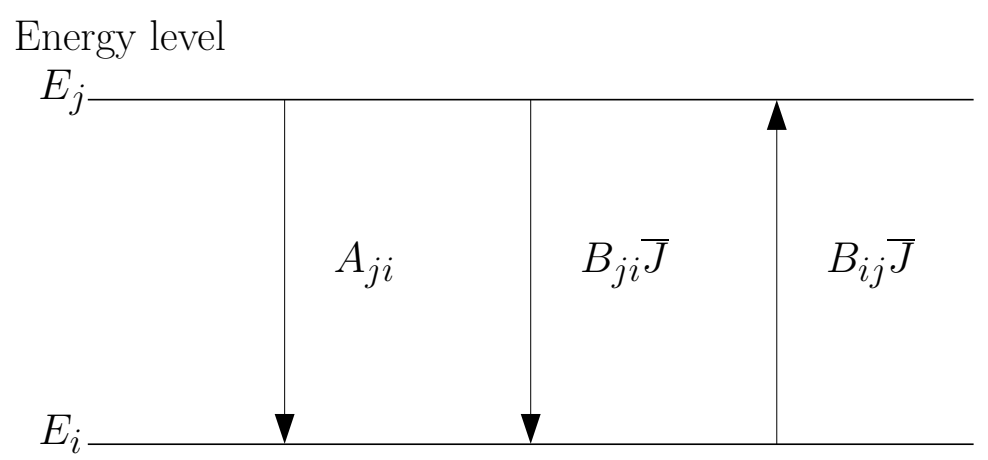

Figure 1.7: Transitions between the energy levels $E_{i}$ and $E_{j}$ of a given species and the Einstein probabilities. Adapted from Wilson et al. (2009).

For a system in a stationary state, the number of transitions per unit time per unit volume out of a given state should meet the number of transitions into this state. Hence, the number of absorbed and emitted photons are equal. Assuming that $n_{i}$ and $n_{j}$ correspond to the number densities of species in levels i and $\mathrm{j}$, respectively, we find the relation (Rybicki and Lightman, 1985):

$$
n_{i} B_{i j} \bar{J}=n_{j} A_{j i}+n_{j} B_{j i} \bar{J}
$$

which in turn imply the following Einstein relations:

$$
\begin{aligned}
& g_{i} B_{i j}=g_{j} B_{j i} \\
& A_{j i}=\frac{2 h \nu^{3}}{c^{2}} B_{j i}
\end{aligned}
$$

where $g_{1}$ and $g_{2}$ are the statistical weights of levels 1 and 2. Equation 1.18 must hold whether or not the system is under thermodynamic equilibrium. In figure 1.7, we present a schematic view of the processes that lead to transitions between the states 1 and 2 of a given species, and their corresponding Einstein coefficients.

\subsection{Rotational transitions}

Among the numerous molecules detected in the gas phase of the ISM, the vast majority of them $(\sim 80 \%)$ has been observed in their rotational lines (McGuire, 2018). Because of the low absolute energy associated with rotational levels, warm interstellar regions (such as hot cores) can provide enough energy to populate those levels, resulting in molecular emission spectra falling in submm/mm wavelengths (e.g., Belloche et al.|2013). Conversely, in colder interstellar environments (such as dark clouds), where there is not enough energy 
to supply a population distribution of the rotational energy levels, molecules can still be observed through absorption transitions against the background continuum (e.g., Neufeld et al. 2012). Thus, radio observations provide the advantage of enabling the search for molecular transitions in nearly any source. Indeed, the remarkable sensitivity and resolution attained by modern radio facilities (e.g., ALMA) result in extremely rich line surveys that represent an unique opportunity to thoroughly explore the chemical inventory of the ISM. Furthermore, the analysis of rotational lines can also give powerful information on the physical properties of the source (e.g., Blake et al. 1987; Goldsmith and Langer 1999), as well as its morphology and evolutionary stage (e.g., Wyrowski et al. 1999; Beuther et al. 2005; Guzmán et al. 2018). In this context, experimental and theoretical rotational spectroscopy share a symbiotic relationship that is fundamental to broaden our percipience of the physics and chemistry of the ISM (see, e.g., Puzzarini 2017; Puzzarini and Barone 2020; Cerqueira et al. 2020; Santos et al. 2020). In order to understand radio observations of molecular lines and its implications on astronomical matters, it is crucial to become acquainted with rotational spectroscopy and its theoretical description. Accordingly, we provide the main concepts involving non-linear rotors in the following subsections.

\subsubsection{The types of rotors}

In classical mechanics, the moment of inertia of a rigid rotor along an arbitrary axis $(\alpha)$ is defined as:

$$
I_{\alpha} \equiv \sum_{i=1}^{n} m_{i} r_{i}^{2}
$$

where $m_{i}$ is the mass of the $i$ th particle and $r_{i}$ is its distance to $\alpha$. Consider a molecule with an axis that passes through its center of mass. In both ends of this axis, we mark a point where the distance to the center of mass corresponds to $I_{\alpha}^{-1 / 2}$. If we repeat the same procedure for the infinite number of axes that pass through the molecule's center of mass, we obtain an ellipsoidal surface known as the "ellipsoid of inertia". The three orthogonal axes of this ellipsoid are called the "principal inertial axes", $a, b$, and $c$, with corresponding moments of inertia $I_{a}, I_{b}$, and $I_{c}$ (Levine, 1975; Hollas, 2004). In Figure 1.8, we show a sketch of the ellipsoid of inertia of $\mathrm{H}_{2} \mathrm{CO}$.

According to their moments of inertia, nonlinear molecules can be classified as spherical 

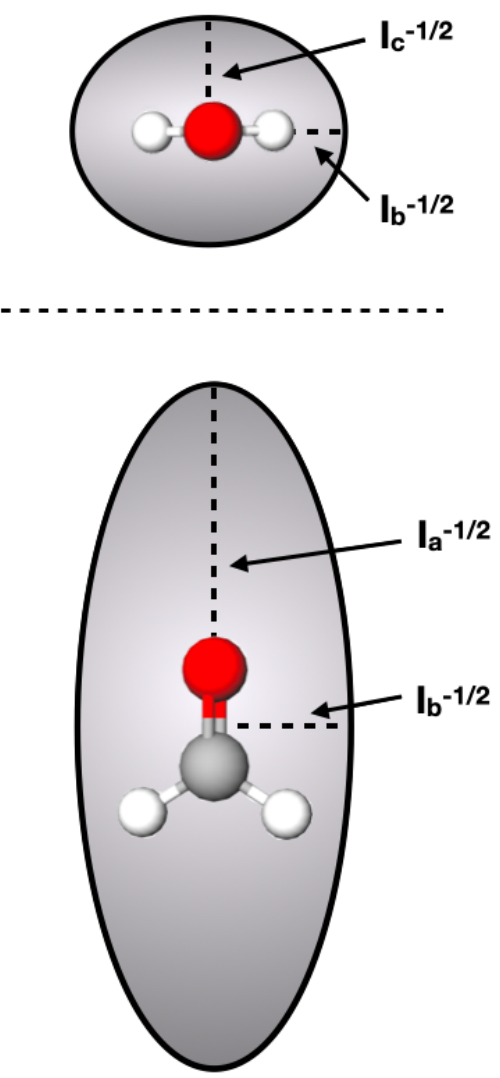

Figure 1.8: Sketch of the ellipsoid of inertia of $\mathrm{H}_{2} \mathrm{CO}$ adapted from Levine (1975). Each cross-section contains two principal axes.

tops $\left(I_{a}=I_{b}=I_{c}\right)$, prolate symmetric tops $\left(I_{a} \neq I_{b}=I_{c}\right)$, oblate symmetric tops $\left(I_{a}=\right.$ $\left.I_{b} \neq I_{c}\right)$, or asymmetric tops $\left(I_{a} \neq I_{b} \neq I_{c}\right)$. Analogously to linear molecules, we can define three rigid rotational constants, $A, B$, and $C$, as:

$$
A \equiv \frac{h}{8 \pi^{2} I_{a}} \geq B \equiv \frac{h}{8 \pi^{2} I_{b}} \geq C \equiv \frac{h}{8 \pi^{2} I_{c}}
$$

where, in the case of asymmetric tops, the $B$ rotational constant can assume any value between $A$ and $C$. Prolate and oblate symmetric tops, therefore, correspond to the limiting cases in which $B=C$ and $B=A$, respectively. In the present thesis, rotational lines of methyl acetylene $\left(\mathrm{CH}_{3} \mathrm{CCH}\right)$, which is a symmetric top, were observed and used to explore the physical conditions of the source. Thus, special focus is given to this type of rotor. For more information on asymmetric tops, the reader is referred to specialized literature (e.g., Santos et al. 2020 and references therein). 


\subsubsection{The rotational Hamiltonian}

The rotational energy of a rigid rotor is classically expressed as:

$$
T_{\text {rot }}=\frac{1}{2}\left(I_{a} \omega_{a}^{2}+I_{b} \omega_{b}^{2}+I_{c} \omega_{c}^{2}\right)
$$

where $\omega_{a}, \omega_{b}$, and $\omega_{c}$ are the angular velocities of the system with respect to the principal molecule-fixed axes $a, b$, and $c$. The rigid-rotor Hamiltonian can therefore be described as a kinetic energy operator expressed in terms of the components of the angular momentum $\hat{J}$ with relation to the principal axes (Gordy and Cook, 1984):

$$
\hat{H}_{\text {rot }}=\frac{1}{2} \frac{\hat{J}_{a}^{2}}{I_{a}}+\frac{1}{2} \frac{\hat{J}_{b}^{2}}{I_{b}}+\frac{1}{2} \frac{\hat{J}_{c}^{2}}{I_{c}}
$$

In an astrochemical context, a highly accurate description of the energy levels is needed in order to properly perform line assignments. Thus, corrections to the rotational Hamiltonian beyond the rigid-rotor and harmonic-oscillator approximations ought to be accounted for (i.e., centrifugal-distortion and vibrational-rotational-coupling effects). This complete Hamiltonian is obtained through a perturbation treatment of the rigid-rotor Hamiltonian, as described elsewhere (Mills 1972; Townes and Schawlow 1975, and references therein). As a result, the rotational Hamiltonian is defined in terms of the zeroth-order rotational constants (A, B, and $\mathrm{C}$ ), and the higher-order centrifugal-distortion constants (e.g., $\mathrm{D}_{J}$, $\mathrm{D}_{J K}, \mathrm{D}_{K}, \mathrm{H}_{J}, \mathrm{H}_{J K}, \mathrm{H}_{K J}$, and $\mathrm{H}_{K}$, for symmetric tops). Those constants are measured from laboratory experiments guided by theoretical simulations (see Puzzarini et al. 2010 and references therein).

The energies of the rotational levels are the eigenvalues of this effective rotational Hamiltonian, which can be obtained by solving the secular equation (Levine, 1975):

$$
\operatorname{det}\left[\left\langle\psi_{m}|\hat{H}| \psi_{n}\right\rangle-E_{i} \delta_{m n}\right]
$$

For symmetric tops, those energy levels are described by two quantum numbers: J, which gives the total angular momentum, and $\mathrm{K}$, which gives the angular momentum component along the molecule-fixed axis c:

$$
\begin{gathered}
\hat{J}^{2}|J, M, K\rangle=J(J+1)|J, M, K\rangle \\
\hat{J}_{c}|J, M, K\rangle= \pm K|J, M, K\rangle
\end{gathered}
$$




\subsubsection{Dipole moment and selection rules}

Rotational transitions are induced by the interaction of the molecule-fixed components of the electric dipole moment with the space-fixed components of the radiation field. Ergo, a permanent electric dipole moment is required in order for the molecule to interact appreciably with the microwave electromagnetic field, resulting in absorption or emission features. The intensities of the rotational transitions are proportional to the square of the dipole moment, which, for symmetric tops, lays along the molecular axis.

The selection rules for dipole radiation of a symmetric top are:

$$
\begin{gathered}
\Delta J=0, \pm 1 \\
\Delta K=0
\end{gathered}
$$

That is to say, the electric dipole matrix elements will be nonzero only for the transitions given by those selection rules (Townes and Schawlow, 1975).

\subsection{Rotational diagrams}

Once believed to be barren, the ISM is nowadays well-known for harboring a myriad of different molecular, ionic, and radical species. With the advent of broadband heterodyne receivers - such as the memorable HIFI instrument on board of Hershel Space Observatory - spectral surveys teeming with rotational lines became wonted. Each one of those transitions is associated with a characteristic critical density and excitation energy, and thereby can be collectively employed as a very effective tool to study the physical properties of their environments. An overview of the probing capabilities of different molecular lines is shown in Figure 1.9

A widely used technique to determine the molecule's excitation temperature and column density consists of constructing rotational diagrams using observed rotational lines (see Goldsmith and Langer 1999). Assuming that $(i)$ the lines are optically thin, (ii) the source is homogeneous and fills the telescope beam, and ( $i i i)$ the emission is thermalized, the observed line intensities can be converted into the upper level column density according to:

$$
N_{u}=\frac{8 \pi k \nu^{2} \int T_{a} d v}{h c^{3} A_{j i}}
$$




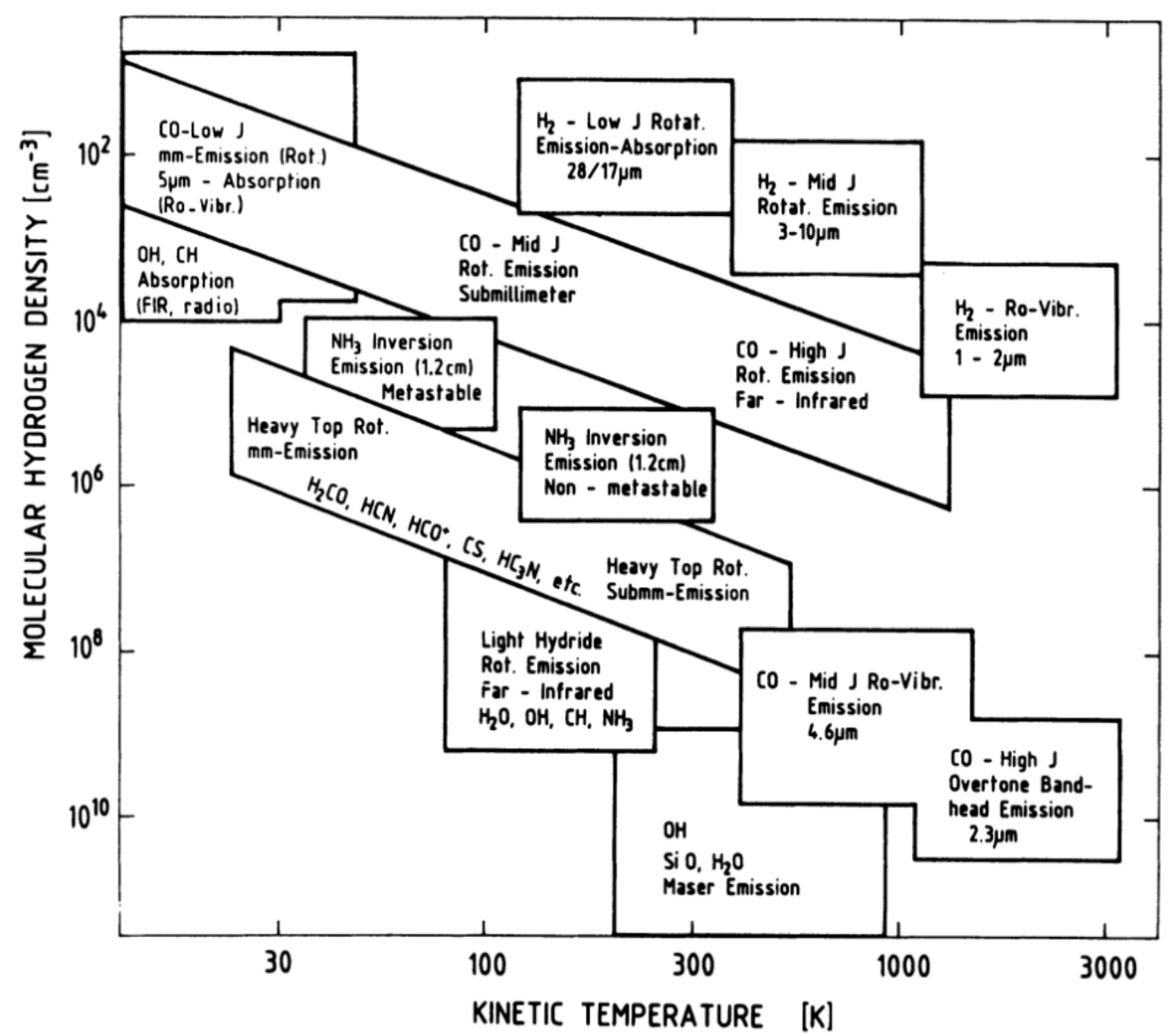

Figure 1.9: Overview of commonly used molecular diagnostics and the respective range of physical conditions that they trace. From Genzel 1991. 
where $\nu$ and $A_{j i}$ are the frequency and the Einstein A coefficient of the transition, respectively. $\int T_{a} d v$ is the integrated intensity of the line. Thus, $\mathrm{N}_{u}$ can be obtained directly from the observed radio spectra.

Under Local Thermodynamic Equilibrium (LTE), the level populations of a given species follow a Boltzmann distribution, and therefore can be characterized by a single excitation temperature $\left(\mathrm{T}_{\text {exc }}\right)$ :

$$
N_{u}=\frac{N_{t o t}}{Q\left(T_{\text {exc }}\right)} g_{u} \exp \left(-\frac{E_{j}}{k T_{\text {exc }}}\right)
$$

where $g_{u}$ is the statistical weight and $\mathrm{E}_{u}$ is the energy of the upper level. $\mathrm{N}_{\text {tot }}$ and $\mathrm{Q}\left(\mathrm{T}_{\text {exc }}\right)$ are the total column density of the given species and its rotational partition function at a temperature $\mathrm{T}_{e x c}$, respectively.

By rewriting Equation 1.27, we derive the expression of the rotational diagram:

$$
\ln \left(\frac{N_{u}}{g_{u}}\right)=\ln \left[\frac{N_{t o t}}{Q\left(T_{e x c}\right)}\right]-\frac{E_{j}}{k T_{e x c}}
$$

Thus, a plot of $\ln \left(N_{u} / g_{u}\right)$ versus $E_{j}$ of the observed transitions will correspond to a straight line, whose slope and y-intercept are defined by $-1 / T_{e x c}$ and $\mathrm{N}_{t o t} / \mathrm{Q}\left(\mathrm{T}_{e x c}\right)$, respectively. For completely thermalized transitions, the excitation temperature derived from the rotational diagram should equal the kinetic temperature $\left(\mathrm{T}_{K}\right)$ of the gas. If the molecule's transitions are not thermalized, however, the level populations deviate from the Boltzmann distribution, and so this approach becomes kaput.

\subsubsection{Opacity correction factor}

In a scenario where the emission has finite values of optical depth, a correction factor $\mathrm{C}_{\tau}$ should be included on the right-hand side of Equation 1.28 Goldsmith and Langer. 1999):

$$
\ln \left(\frac{N_{u}}{g_{u}}\right)=\ln \left[\frac{N_{t o t}}{Q\left(T_{e x c}\right)}\right]-\ln C_{\tau}-\frac{E_{j}}{k T_{e x c}}
$$

in which $\mathrm{C}_{\tau}$ corresponds to a photon escape probability formulation:

$$
C_{\tau}=\frac{\tau}{1-e^{-\tau}}
$$


For the limiting case of $\tau \ll 1$ (i.e., the optically thin scenario), $C_{\tau} \simeq 1$ and Equation 1.29 reduces to 1.28 . Hence, if the emission is mistakenly assumed to be optically thin, each of the upper level populations will be underestimated by a factor $\mathrm{C}_{\tau}$, which will affect the derived $N_{\text {tot }}$. Moreover, since $\tau$ (and therefore $\mathrm{C}_{\tau}$ ) vary from line to line, the slope of the diagram will also be affected, leading to overestimated values of $\mathrm{T}_{\text {exc }}$.

\subsubsection{Beam dilution factor}

For point-like sources, the assumption that the telescope beam is filled by the emission is preposterous. In this case, Equation 1.29, must be corrected by the so-called "beamdilution factor" $\left(\Delta \Omega_{a} / \Delta \Omega_{s}\right)$ :

$$
\ln \left(\frac{N_{u}}{g_{u}}\right)=\ln \left[\frac{N_{t o t}}{Q\left(T_{\text {exc }}\right)}\right]-\ln C_{\tau}-\ln \left(\frac{\Delta \Omega_{a}}{\Delta \Omega_{s}}\right)-\frac{E_{j}}{k T_{\text {exc }}}
$$

where $\Delta \Omega_{a}$ and $\Delta \Omega_{s}$ are, respectively, the beam solid angle and the source solid angle. In other words, when the emitting region does not fill the beam, the observed antenna temperatures will be systematically reduced by the beam dilution factor. This effect, in turn, will also influence the inferred optical depths. Moreover, for broad surveys encompassing a wide range of frequencies, such as the present work, the dilution factor does not remain constant for the whole spectral range. As a result, not only the derived $\mathrm{N}_{t o t}$ but also $\mathrm{T}_{\text {exc }}$ are affected by this correction-or lack thereof.

\subsection{The spectral tracer methyl acetylene}

The present thesis revolves around the observed rotational lines of a particularly fruitful molecule: methyl acetylene $\left(\mathrm{CH}_{3} \mathrm{CCH}\right)$. This species is a prolate symmetric rotor that pertains to the point group $\mathrm{C}_{3} \mathrm{v}$, and therefore its rotational transitions are characterized by the quantum numbers $\mathrm{J}$ and $\mathrm{K}$ (see $\$ 1.5$ ). At standard temperature and pressure, it is a very flammable and reactive ga:5. A 3D model of this molecule is presented In Figure 1.10, together with other important molecular tracers.

The earliest tentative detection of $\mathrm{CH}_{3} \mathrm{CCH}$ in the interstellar medium (ISM) was reported by Buhl and Snyder (1973), toward the Sgr B2 molecular cloud. Later, Lovas

${ }^{4}$ https://cccbdb.nist.gov/pglist.asp

${ }^{5}$ https://pubchem.ncbi.nlm.nih.gov/compound/Propyne 


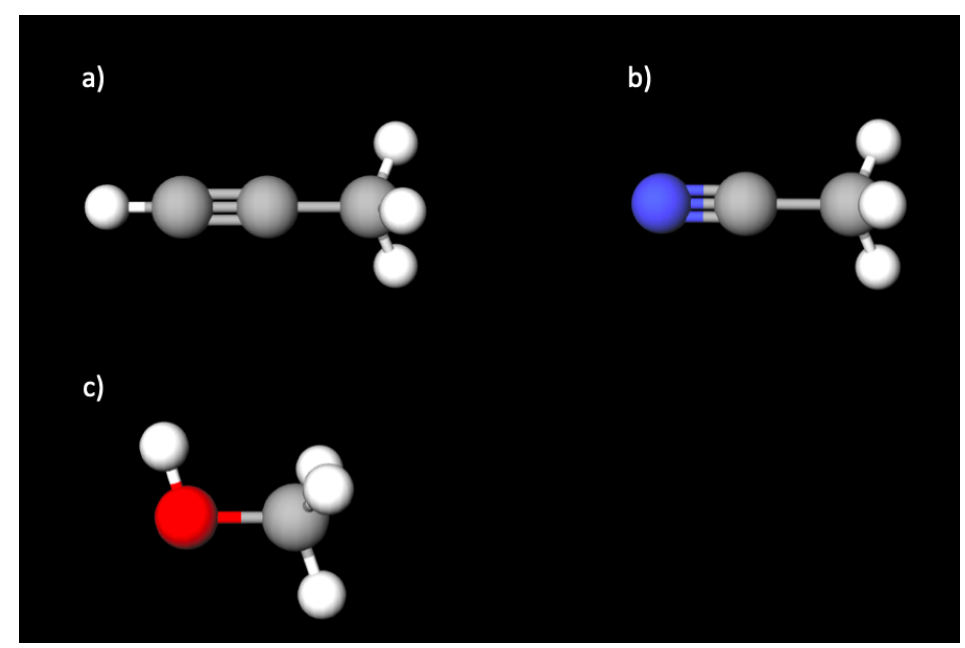

Figure 1.10: 3D models of the molecules $\mathrm{CH}_{3} \mathrm{CCH}, \mathrm{CH}_{3} \mathrm{CN}$ and $\mathrm{CH}_{3} \mathrm{OH}$, for comparison purposes. Symmetric rotors: a) methyl acetylene $\left(\mathrm{CH}_{3} \mathrm{CCH}\right)$ and b) acetonitrile $\left(\mathrm{CH}_{3} \mathrm{CN}\right)$. Asymmetric rotor: c) methanol $\left(\mathrm{CH}_{3} \mathrm{OH}\right)$. The white, gray, blue and red spheres correspond to the $\mathrm{H}, \mathrm{C}, \mathrm{N}$ and $\mathrm{O}$ atoms, respectively. Those three molecules have been observed in G331 (Hervías-Caimapo et al., 2019), and play an important role in the inference of the physical conditions of HMCs and related sources.

et al. (1976) observed $\mathrm{CH}_{3} \mathrm{CCH}$, among other molecules containing the methyl group (e.g., $\mathrm{CH}_{3} \mathrm{OH}, \mathrm{CH}_{3} \mathrm{CN}$ and $\mathrm{CH}_{3} \mathrm{CHO}$ ), toward the Orion A region. In the following years, Hollis et al. (1981) performed a survey toward Sgr B2 and recorded new observations of $\mathrm{CH}_{3} \mathrm{CCH}$ that confirmed Buhl \& Snyder's detection. Similarly, Kuiper et al. (1984) conducted a successful search for $\mathrm{CH}_{3} \mathrm{CCH}$ in Sgr B2, as well as in various other dense interstellar clouds. Since its first detection, $\mathrm{CH}_{3} \mathrm{CCH}$ has been extensively studied toward star-forming regions (e.g., Zhang et al. 2014; Taniguchi et al. 2018; Guzmán et al. 2018; Bøgelund et al. 2019; Calcutt et al. 2019), and has proven to be a reliable tracer of their physical conditions (e.g., Bergin et al. 1994). It has also been observed toward extagalactic sources, such as M 82, NGC 253, and NGC 1068 (Mauersberger et al., 1991; Qiu et al., 2020), and even toward a planetary nebula, as recently reported by Schmidt and Ziurys (2019). As a matter of fact, the widespread detection of $\mathrm{CH}_{3} \mathrm{CCH}$ even extends to planetary atmospheres (e.g., de Graauw et al. 1997; Fouchet et al. 2000; Burgdorf et al. 2006; Cordiner et al. 2015).

Formation pathways of $\mathrm{CH}_{3} \mathrm{CCH}$ in the gas-phase have been extensively investigated. Ion-neutral routes with $\mathrm{C}_{2} \mathrm{H}_{2}^{+}$as a precursor Schiff and Bohme, 1979, Millar and Freeman, 1984), neutral-neutral reactions such as $\mathrm{CCH}+\mathrm{CH}_{4} \rightarrow \mathrm{CH}_{3} \mathrm{CCH}+\mathrm{H}$ (Turner et al., 1999), as well as dissociative recombination reactions involving larger hydrocarbons (Calcutt et al., 2019) are all subsumed under the suggested gaseous formation routes of $\mathrm{CH}_{3} \mathrm{CCH}$. However, those pathways alone fail to reproduce the observed abundances in 
dense molecular clouds (Li et al., 2013; Öberg et al., 2013, Vastel et al., 2014). For this reason, grain-surface reactions are additionally proposed as formation routes (Hickson et al. 2016; Guzmán et al. 2018). If this is the case, however, its solid-phase formation and consecutive desorption mechanisms should be efficient at temperatures as low as $\sim 20 \mathrm{~K}$, as pointed out by Giannetti et al. (2017). Moreover, Qasim et al. (2019) have shown that solid-phase methyl acetylene can play an important role as a precursor of alcohols under dark-cloud conditions. Using a combination of experiments and theoretical chemistry, they found that n-propanol $\left(\mathrm{CH}_{3} \mathrm{CH}_{2} \mathrm{CH}_{2} \mathrm{OH}\right)$ and isopropanol $\left(\left(\mathrm{CH}_{3}\right)_{2} \mathrm{CHOH}\right)$ were efficiently formed at low temperatures $(\mathrm{T}=10 \mathrm{~K})$ and without any energy input (such as UV radiation or cosmic rays), through the radical-addition reaction of $\mathrm{CH}_{3} \mathrm{CCH}+\mathrm{OH}$.

Because it is a symmetric rotor, the rotational levels of $\mathrm{CH}_{3} \mathrm{CCH}$ with a given $\mathrm{J}$ quantum number are divided into multiple levels with different $\mathrm{K}$ quantum numbers (see Figure 1.11). The effects of centrifugal distortion lead to small energy differences between each K level, splitting their degeneracy. As a result, for each $\mathrm{J}$ transition, there are many lines with different $\mathrm{K}$ quantum numbers encompassing a wide range of energies above the ground state. Those different $\mathrm{K}$ lines with the same J quantum numbers - the socalled K-ladders - are closely spaced in frequency, and therefore can all be observed in the same bandwidth. This procedure has the advantage of reducing calibration uncertainties, thus yielding more precise predictions. Moreover, due to its small electric dipole moment $\left(\mu=0.75 \mathrm{D}\right.$, Dubrulle et al. 1978), line thermalization occurs at densities as low as $\sim 10^{4}$ $\mathrm{cm}^{-3}$ (e.g., Bergin et al. 1994; Fontani et al. 2002; Molinari et al. 2016), well below the expected density at G331 $\left(n\left(\mathrm{H}_{2}\right) \sim 10^{7} \mathrm{~cm}^{-3}\right.$, Mendoza et al. 2018). Thus, the LTE requirement for the rotational diagram analysis (see \$1.6) is easily met. Furthermore, since the dipole moment for symmetric tops lays parallel to the symmetry axis, radiative transitions with $\Delta K \neq 0$ are forbidden. As a result, the relative population of the K-ladders are dictated by the kinetic temperature of the colliding particles, which makes $\mathrm{CH}_{3} \mathrm{CCH}$ an excellent temperature probe.

This molecule is generally observed to have an extended emitting region and to trace relatively cold gas (Fontani et al., 2002; Bisschop et al., 2007; Giannetti et al., 2017; Andron et al., 2018). It is also highly sensitive to the warm-up process that takes place during the development of a hot core (Giannetti et al., 2017). Indeed, methyl acetylene provides a cornucopia of powerful information on the physics and chemistry of the environment. Its 


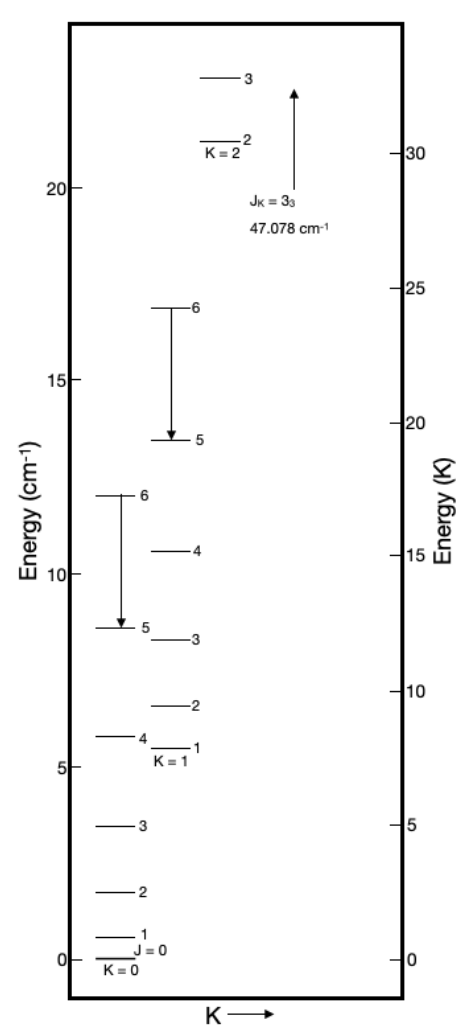

Figure 1.11: Rotational energy levels of $\mathrm{CH}_{3} \mathrm{CCH}$. Adapted from Kuiper et al. (1984).

temperature probing potential can go even beyond the statistically-averaged, large-scale analysis obtained through rotational diagrams. Rather, as is going to be elucidated along this thesis, the intrinsic spectroscopic properties of $\mathrm{CH}_{3} \mathrm{CCH}$ allows not only to assess the overall temperature of the source, but also to explore its small scale temperature profile even with single-dish data. 
Chapter 2

\section{Facilities and methods}

In this chapter, we outline the different steps executed throughout this research project to explore the excitation conditions of $\mathrm{CH}_{3} \mathrm{CCH}$ in the $\mathrm{HMC}$ G331. Those include: information on the facilities, observations, and subsequent data reduction procedures, a description of the line-fitting and LTE-analysis techniques, as well as an overview of the theoretical-chemistry methods that were employed in order to further investigate the observed spectra. Since the relevant theoretical background has been addressed in the previous chapter (see \$1), we will hereupon focus on a pragmatic approach to the routines and technicalities associated with those methods (e.g., software, keywords, and other apposite details).

\subsection{The Atacama Pathfinder EXperiment (APEX)}

In the present work, the physical properties of G331 were explored using molecular line observations from the APEX radio telescope. For this reason, we now give a brief introduction on this facility. See Figure 2.1 for a prepossessing picture of the antenna.

The 12 m Atacama Pathfinder EXperiment (APEX) telescope is located at Llano de Chajnantor, in northern Chile, at an altitude of $\sim 5100 \mathrm{~m}$ above the sea level. It is a collaboration between the Max Planck Institut für Radioastronomie (45\% observing time), the European Southern Observatory (24\%), and the Onsala Space Observatory (21\%). As the host country for the project, Chilean research institutions have access to $10 \%$ of the available observing time.

APEX is based on an ALMA prototype antenna modified to suit single-dish operations. It was manufactured by VERTEX Antennentechnik GmbH, in Germany, and started 


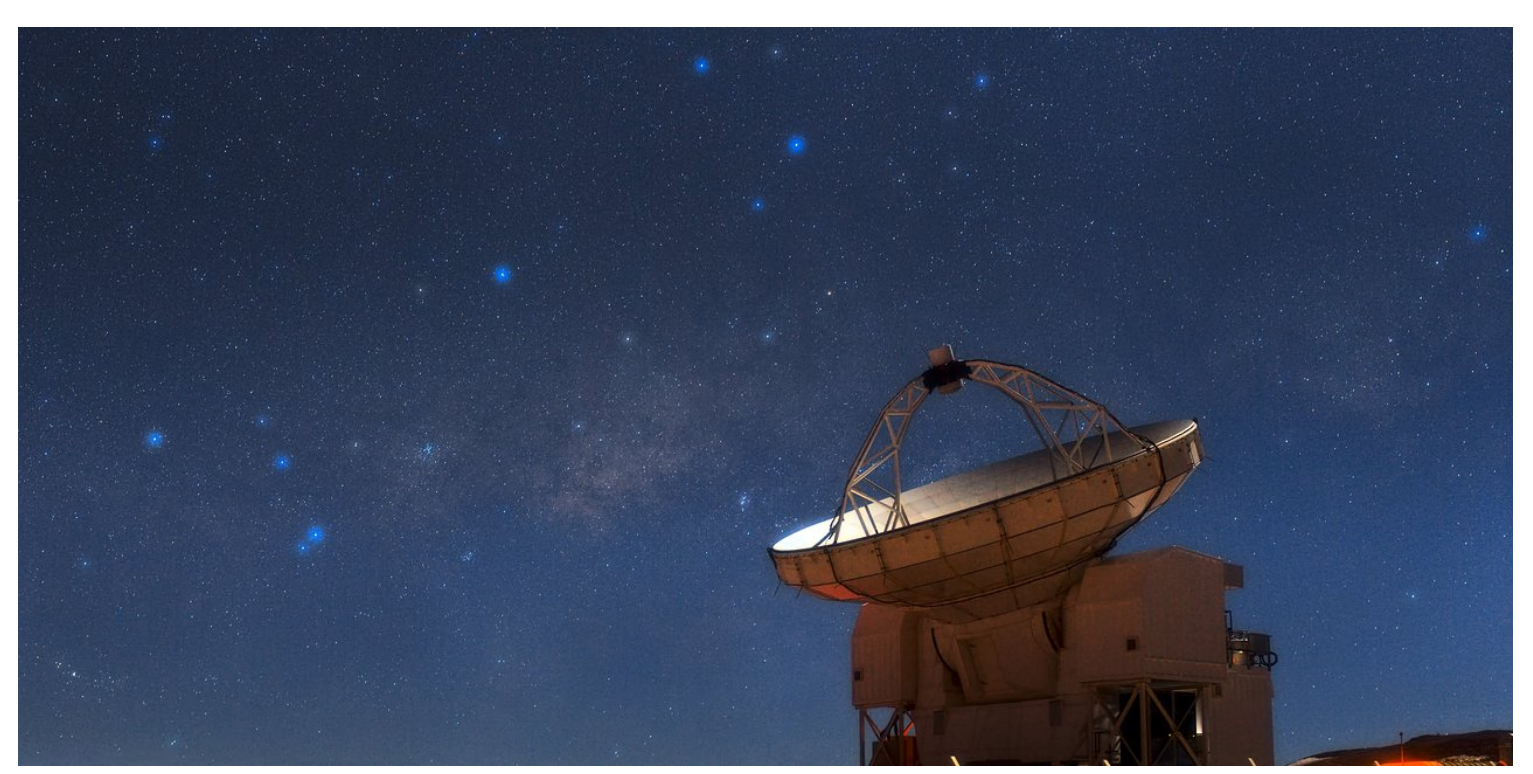

Figure 2.1: APEX $12 \mathrm{~m}$ telescope. Credit: ESO/B. Tafreshi

operating in 2005. The telescope is built over an alt-azimuthal mount, and consists of a Cassegrain system with a paraboloidal main reflector. The effective surface accuracy is calculated to be $\sim 17 \mu \mathrm{m}$ rms (Güsten et al., 2006). Because of the high altitude, the amount of Precipitable Water Vapor (PWV) at the APEX site is typically $1.0 \mathrm{~mm}$ and falls below $0.5 \mathrm{~mm} \leq 25 \%$ of the time ${ }^{1}$

The instrumentation programme at APEX is very extense and diverse. Multiple facilities are available in order to cover a broad range of scientific purposes. In the present work, two different instruments were used to conduct our spectral survey of $\mathrm{CH}_{3} \mathrm{CCH}$ toward G331: the Swedish Heterodyne Facility Instrument (SHeFI) and the Swedish-ESO PI Instrument (SEPIA). General information on those insruments are given below.

\subsubsection{SHeFI}

Decommissioned since 2017, the Swedish Heterodyne Facility Instrument (SHeFI) consisted of four wide-band heterodyne receiver channels located in the Nasmith cabin A of APEX. Since SHeFI only allows observations with one frequency band at a time, a channelswitching mirror directs the beam into a specific channel window. The coupled cryostat is equipped with a liquid He pot for temperature stabilization, and yields temperatures of $\sim 3.6 \mathrm{~K}$.

Collectively, the four bands of SHeFI covered a frequency range between 211 and 1390

\footnotetext{
1 http://www .apex-telescope.org/ns/weather/
} 
GHz. Their information are summarized in Table 2.1.2 For more details on the instrument configuration and on each particular channel, see Vassilev et al. (2008).

Table 2.1 - Frequency channels of the SHeFI instrument. SIS stands for superconductor-insulatorsuperconductor mixer; SSB stands for single sideband receiver; HEB stands for hot electron bolometer mixer; and DSB stands for double sideband receiver.

\begin{tabular}{cccc}
\hline \hline $\begin{array}{c}\text { APEX } \\
\text { Band }\end{array}$ & $\begin{array}{c}\text { Frequency } \\
\text { GHz }\end{array}$ & Technology & $\begin{array}{c}H P B W \\
\operatorname{arcsec}\end{array}$ \\
\hline 1 & $213-275$ & SIS,SSB & $30-23$ \\
2 & $267-378$ & SIS,SSB & $23-17$ \\
3 & $385-506$ & SIS,DSB & $17-13$ \\
4 & $1250-1390$ & HEB, DSB & 5 \\
\hline \hline
\end{tabular}

\subsubsection{SEPIA}

The Swedish-ESO PI Instrument (SEPIA) was brought to APEX in the beginning of 2015 and installed in Cabin A. At the moment, it accommodates three ALMA-like heterodyne receivers (the so-called "cartridges"): SEPIA660, SEPIA345, and SEPIA180. Their information is summarized in Table $2.2{ }^{3}$

Table 2.2 - Frequency channels of the SEPIA instrument. SIS stands for superconductor-insulatorsuperconductor mixer and 2SB stands for dual sideband receiver.

\begin{tabular}{cccc}
\hline \hline SEPIA & $\begin{array}{c}\text { Frequency } \\
\text { GHz }\end{array}$ & Technology & $\begin{array}{c}H P B W \\
\text { arcsec }\end{array}$ \\
\hline 660 & $597-725$ & SIS,2SB & $10-9$ \\
345 & $272-376$ & SIS,2SB & $23-17$ \\
180 & $157-211$ & SIS,2SB & $39-30$ \\
\hline \hline
\end{tabular}

Similarly to SHeFI, only one receiver can be used at a given time. However, this issue is partially mitigated by the fast switching time between receivers (of only a few minutes). More information on this instruments, and particularly on the SEPIA180 receiver-also known as SEPIA-B5 - can be found in Belitsky et al. (2018).

\footnotetext{
2 http://www. apex-telescope.org/heterodyne/shfi/

3 http://www . apex-telescope.org/ns/instruments/sepia/
} 


\subsection{Observations and data calibration}

The observations were obtained with APEX 12 m telescope (Güsten et al., 2006) (see $\$ 2.1$, toward the coordinates RA, DEC $=16^{h} 12^{m} 10.1^{s},-51^{\circ} 28^{\prime} 38.1^{\prime \prime}$. We employed the Band 5 receiver of the Swedish-ESO PI Instrument (SEPIA-B5; Belitsky et al. 2018), together with APEX-1 and APEX-2 receivers of the Swedish Heterodyne Facility Instrument (SHeFI; Vassilev et al. 2008), to observe nine frequency setups within the intervals 170.8205.1 GHz and 222-307.6 GH, respectively. As backend, the eXtended bandwidth Fast Fourier Transform Spectrometer2 (XFFTS2) was used, which consists of two units with 2.5 GHz bandwidth divided into 32768 channels each. The spectral resolution, corresponding to velocity resolutions, was obtained between 0.15 and $0.25 \mathrm{~km} \mathrm{~s}^{-1}$ for a noise level of $\sim 30-50 \mathrm{mK}$.

The signal received from the sky is converted from the telescope count units to units of antenna temperature $\mathrm{T}_{A}$ by observing a load with controlled temperature and comparing it to observations of the sky. $\mathrm{T}_{A}$ is generally given by the expression (Milligan, 2005):

$$
T_{A}=\frac{1}{4 \pi} \int_{0}^{2 \pi} \int_{0}^{\pi} G(\theta, \varphi) T_{S}(\theta, \varphi) \sin (\theta) d \theta d \varphi
$$

where $G(\theta, \varphi)$ is the antenna's gain pattern and $T_{S}(\theta, \varphi)$ is the temperature distribution of the environment.

Atmospheric models are employed to aid the correction for atmospheric effects Dumke and Mac-Auliffe, 2010). Moreover, the sky background is subtracted from the signal by means of the ON/OFF switching mode, in which the pointing direction of the antenna main-beam is switched repeatedly between an ON position and a reference position. This OFF position should not be too far (in terms of celestial coordinates) from the ON position. Thus, assuming that the broadband emission from the sky, ground, and/or continuum sources are similar at both ON and OFF positions, spatial and temporal signal fluctuations are canceled.

The antenna temperature, $\mathrm{T}_{A}$, is then converted to the main-beam brightness temperature by: $:^{4}$

$$
T_{m b}=T_{A} \frac{\eta_{f w}}{\eta_{m b}}
$$

\footnotetext{
4 http://www . apex-telescope.org/telescope/efficiency/
} 
where $\eta_{f w}$ and $\eta_{m b}$ are the forward and main beam efficiencies, respectively. For all observations, $\eta_{f w}=0.95$, whereas $\eta_{m b}=0.82$ for SEPIA-B5 Galametz et al., 2016; Zahorecz et al. 2017), and $\eta_{m b}=0.75$ and 0.73 for APEX-1 and APEX-2, respectively (Mendoza et al., 2018; Duronea et al., 2019). The Half Power Beam Width (HPBW) varies between $\sim 17-39^{\prime \prime}$ for the entire frequency range. We adopted a calibration uncertainty of about $10 \%$ (Dumke and Mac-Auliffe, 2010), and a surface accuracy (rms) of $17 \mu \mathrm{m}:^{5}$

\subsection{Data reduction}

The data reduction was carried out using the CLASS package of the Grenoble Image and Line Data Anlysis Software (GILDAS).$^{6}$ This software has been adopted as the standard reduction package for APEX, and consists of five programs with different utilities:

- CLASS (Continuum and Line Analysis Single-dish Software), dedicated to processing single-dish data;

- GREG (Grenoble Graphic), dedicated to 1-D and 2-D imaging;

- ASTRO, dedicated to preparing observing sessions;

- CLIC (Continuum and Line Interferometric Calibration), dedicated to calibrating data from the NOEMA interferometer;

- Mapping, dedicated to interactive imaging and deconvolution.

First, the observations were inspected and those with calibration errors were discarded. Then, first-degree polynomial baselines were removed from each individual scan, by means of the base command. Finally, the baseline-corrected observations were averaged into a final spectrum using the stitch command.

\subsection{Line identification and gaussian fitting}

Line identifications were performed using the Weeds extension of CLASS, in combination with spectroscopic databases such as NIST7 Recommended Rest Frequencies for

\footnotetext{
5 http://www. apex-telescope.org/telescope/

6 https://www.iram.fr/IRAMFR/GILDAS/

7 https://physics.nist.gov/cgi-bin/micro/table5/start.pl
} 
Observed Interstellar Molecular Microwave Transitions (Lovas, 2004), CDMS 8 (Endres et al. 2016), JPL (Pickett et al., 1998), and Splatalogue ${ }^{10}$, The following criteria were defined to perform the identification:

- The peak frequencies of the observed lines should be consistent with $\mathrm{V}_{l s r} \sim-90$ $\mathrm{km} \mathrm{s}^{-1}$ (the systemic velocity of G331);

- The intensities of the observed lines should surpass the threshold of $3 \sigma$;

- Lines predicted through LTE modeling should agree with the observations to a reasonable extent.

The last requirement of the list involves a well defined rotational temperature - which is obtained through a rotational diagram analysis - and thus will be discussed in $\$ 2.5 .2$. In addition to Weeds, the CASSIS11 software was used to confirm the detected lines. Moreover, we also inspected the identified lines for contamination by adjacent molecular transitions, by means of the line search engine within Weeds.

The spectroscopic properties of the lines were obtained through fitting a single gaussian profile to each line. This was performed by the method gauss command, whose fitted parameters are: area, position, and width. For features not completely resolved, the single gaussian fitting was ensured by means of the set mask keyword, which selects regions of the spectrum to be excluded from the fit. An example of this procedure is shown in Figure 2.2, where the observed $\mathrm{CH}_{3} \mathrm{CCH}\left(10_{0}-9_{0}\right)$ line is depicted together with its gaussian fitting.

\subsection{LTE analyses}

\subsubsection{Rotational diagrams}

The rotational diagram analysis (see $\$ 1.6$ ) was carried out with CASSIS. In order to properly infer $\mathrm{T}_{\text {exc }}$ and $\mathrm{N}_{t o t}$, the error bars of the points in the diagram must be taken into account when fitting the $1^{\text {st }}$ order polynomial. The integrated area uncertainty is given by:

\footnotetext{
$8 \longdiv { \text { https://cdms.astro.uni-koeln.de/ } }$

9 https://spec.jpl.nasa.gov/

10 https://www.cv.nrao.edu/php/splat/

11 http://cassis.irap.omp.eu/
} 


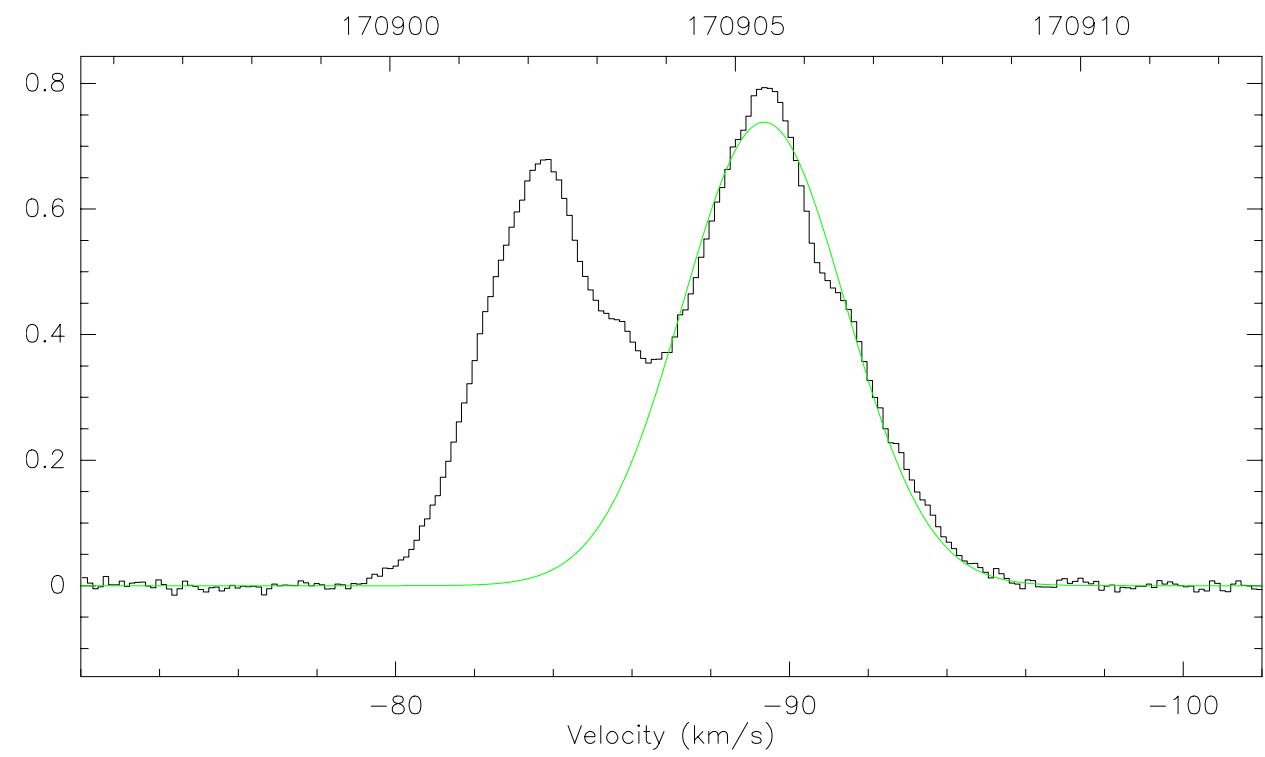

Figure 2.2: Gaussian fitting of the $\mathrm{CH}_{3} \mathrm{CCH}\left(10_{0}-9_{0}\right)$ line (green) over the observed spectrum (black). The upper and lower $\mathrm{x}$-axes are given in frequency units $(\mathrm{MHz})$ and velocity units $\left(\mathrm{km} \mathrm{s}^{-1}\right)$, respectively. The $\mathrm{y}$-axis is given in temperature units $(\mathrm{K})$.

$$
\Delta W=\sqrt{(c a l / 100 \times W)^{2}+(r m s \sqrt{2 \times F W H M \times \Delta v})^{2}}
$$

where $\mathrm{W}$ is the integrated area $\left(\mathrm{K} \mathrm{km} \mathrm{s}^{-1}\right)$, rms is the noise around the selected species $(\mathrm{K})$, FWHM is the fitted Full Width at Half Maximum $\left(\mathrm{km} \mathrm{s}^{-1}\right), \Delta v$ is the bin size $(\mathrm{km}$ $\left.\mathrm{s}^{-1}\right)$, and cal is the calibration value $(\sim 10 \%)$. The plotted uncertainties are, thus:

$$
\Delta\left(\ln \frac{N_{u}}{g_{u}}\right)=\frac{\Delta W}{W}
$$

The uncertainties on the fitted parameters $\left(\mathrm{T}_{\text {exc }}\right.$ and $\left.\mathrm{N}_{t o t}\right)$ are related, respectively, to the slope (a) and the $y$-intercept (b) of the fitted polynomial (i.e., $y=a x+b$ ). Since $\mathrm{T}_{\text {exc }}=-1 / \mathrm{a}$ and $\mathrm{N}_{t o t}=Q\left(\mathrm{~T}_{\text {exc }}\right) \times e^{b}$, it yields:

$$
\begin{gathered}
\Delta T_{e x c}=\frac{\Delta a}{a^{2}} \\
\Delta N_{t o t}=Q\left(T_{e x c}\right) \times \Delta b \times e^{b}
\end{gathered}
$$

The opacity correction (see $\$ 1.6 .1$ is incorporated iteratively by means of Equations 1.28 and 1.29 , until a solution for $\mathrm{T}_{\text {exc }}$ and $\mathrm{N}_{\text {tot }}$ converges. The convergence criterion was defined as $\epsilon=1 \%$ - that is to say, the values are deemed converged when the difference between the last two iterations is smaller than $1 \%$. 
The quality of the fit can be assessed through its reduced $\chi^{2}$ values, in which the degrees of freedom correspond to:

$$
d o f=n-2
$$

where $\mathrm{n}$ is the number of points on which the fit was performed. Since two values are extracted from the fit $\left(\mathrm{T}_{\text {exc }}\right.$ and $\left.\mathrm{N}_{t o t}\right)$, we must subtract 2 from $\mathrm{n}$. Thus, $\chi_{\text {red }}^{2}$ is given by:

$$
\chi_{\text {red }}^{2} \times d o f=\sum_{i=1}^{n} \frac{\left(y_{i}-y\right)^{2}}{\sigma}
$$

where $\sigma$ corresponds to the uncertainty of the points (see Equation 2.4). The closer $\chi_{\text {red }}^{2}$ is to 1 , the better is the fit.

\subsubsection{Synthetic LTE spectra}

The Weeds extension of CLASS allows to simulate molecular emission under the assumption of LTE (see Maret et al. 2011). The input parameters for each species are $\mathrm{T}_{\text {exc }}$, $\mathrm{N}_{\text {tot }}$, FWHM, as well as the source's systemic velocity and size. The LTE spectrum of a given species is computed by:

$$
T_{B}(\nu)=\eta\left[J_{\nu}\left(T_{e x c}\right)-J_{\nu}\left(T_{b g}\right)\right]\left(1-e^{-\tau(\nu)}\right)
$$

where $\eta$ is the beam dilution factor (see 1.6.2), $\mathrm{T}_{b g}$ is the brightness temperature of the background emission (e.g., $2.73 \mathrm{~K}$ for the Cosmic Microwave Background (CMB)), and $\tau(\nu)$ is the opacity. The modeled lines can thus appear in absorption or emission, depending on the continuum and line excitation temperatures. Multiple species and/or components can be computed simultaneously, resulting in a spectrum that is the sum of the brightness temperature of each species/component given by Equation 2.8. In this case, it is assumed that the different species/components are not coupled radiatively, viz. one species/component will not absorb photons from another species/component.

The resulting model should, therefore, closely resemble the observed spectrum - provided the LTE assumption is met. For emission features, the background is computed as the sum of the CMB and the source continuum emission, which is assumed to be optically thin. Moreover, the continuum is presupposed to be originated from a location behind the medium where the lines are produced. 


\subsection{Rotational-spectrum simulations}

Aside from radio-astronomical techniques, the present work also utilizes quantumchemistry methods to explore the observations of $\mathrm{CH}_{3} \mathrm{CCH}$ in a molecular level. The rotational spectrum of $\mathrm{CH}_{3} \mathrm{CCH}$ was simulated at various temperatures using the PGOPHER (Western, 2017) general purpose software, which performs the diagonalization of the appropriate Hamiltonian matrix to yield the rotational energy levels (see 1.5 ).

For symmetric tops, such as $\mathrm{CH}_{3} \mathrm{CCH}$, the rotational Hamiltonian is standard $(\mathrm{Pa}-$ pousek and Aliev, 1982), and yields ground-vibrational-state rotational energies according to:

$$
\begin{aligned}
E_{J, K}= & B J(J+1)+(A-B) K^{2}-D_{J} J^{2}(J+1)^{2}-D_{J K} J(J+1) K^{2}-D_{K} K^{4} \\
& +H_{J} J^{3}(J+1)^{3}+H_{J K} J^{2}(J+1)^{2} K^{2}+H_{K J} J(J+1) K^{4}+H_{K} K^{6}
\end{aligned}
$$

The experimental rotational constants, centrifugal distortion constants, and dipole moment used in the simulations were taken from the JPL 12 database. The transition intensities are given by (Western, 2017):

$$
I=\frac{S}{Q(T)}\left[\exp \left(-\frac{E_{i}}{k T}\right)-\exp \left(-\frac{E_{j}}{k T}\right)\right]
$$

which is simply the line strength $S$ times the Boltzmann factor, normalized to the temperaturedependent rotational partition function $Q(T)$ at the given temperature. $E_{i}$ and $E_{j}$ correspond to the energies of the lower and the upper states, respectively.

The temperature-dependent partition function for a molecule, $Q(T)$, is defined as:

$$
Q(T)=\sum g_{i} e^{-E_{i} / k T}
$$

where $g_{i}$ is the statistical weight and $E_{i}$ is the energy of the $i$ th state. The computation is assumed to have converged when the highest $\mathrm{J}$ values have a contribution of $<10^{-4}$ to the overall sum.

\footnotetext{
12 https://spec.jpl.nasa.gov/
} 
Chapter 3

\section{Results and discussion}

\subsection{Line properties}

In the present thesis, we have conducted a spectral survey of methyl acetylene toward the HMC G331. In total, 41 uncontaminated lines of $\mathrm{CH}_{3} \mathrm{CCH}$ were detected within a frequency range from $\sim 170840 \mathrm{MHz}$ to $\sim 307600 \mathrm{MHz}$. In other words, all the detected lines satisfy the criteria listed in $\$ 2.4$ and no plausible line contamination was identified nearby the transitions. Those lines are distributed in nine different K-ladders, ranging from the $\mathrm{J}=10-9$ to $\mathrm{J}=18-17$ rotational levels, all of which are displayed in Figure 3.1 . For all 41 detected lines, the ratios of line intensity to baseline rms were $\gtrsim 5.2$, well above the $3 \sigma$ threshold. For example, the baseline reduction of the $\mathrm{J}=10_{K}-9_{K}$ K-ladder yielded an rms of $\sim 5.8 \times 10^{-3} \mathrm{~K}$, whereas the intensity of the $\mathrm{J}=10_{4}-9_{4}$ transition-which is the faintest detected line within this K-ladder - corresponds to $\sim 6.3 \times 10^{-2} \mathrm{~K}$. The resulting intensity/rms ratio is $\sim 10.8$. Such a large number of detected lines provide an unique opportunity to explore the physical conditions of G331 with remarkably robust statistics. Isotopologue transitions were not unequivocally identified and therefore were not considered in our analyses.

Spectroscopic parameters obtained through single gaussian fittings of the lines are summarized in Table 3.1. In reality, most lines profiles require more components to be fitted accurately (e.g., Roberts et al. 2011; Galván-Madrid et al. 2013), warranting more demanding line fitting procedures. This level of sophistication, however, is beyond the scope of this work - for our purposes, a simplified approach suffices. For each observed $\mathrm{J}_{u}$ level, all transitions with $\mathrm{K}=3-0$ were detected. In some cases (see Table 3.1), lines with $\mathrm{K}=4$ were not detectable above the 3 sigma threshold. Nevertheless, all K-ladders 

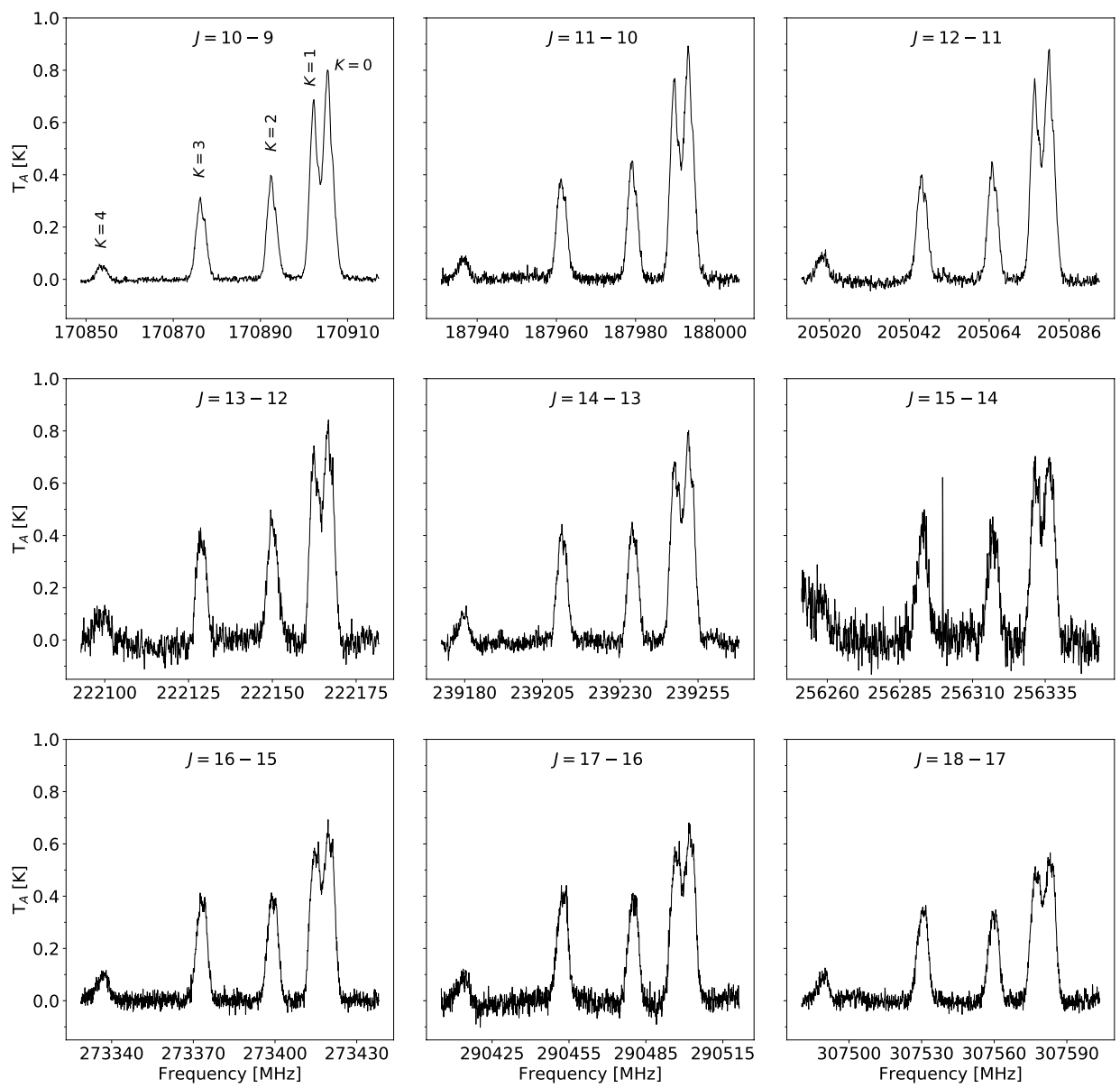

Figure 3.1: Spectra of $\mathrm{CH}_{3} \mathrm{CCH}$ exhibiting the different K-ladders identified from $\mathrm{J}=10-9$ to $\mathrm{J}=18-17$. For each spectrum, a linear baseline was subtracted. K-level transitions are indicated for the $\mathrm{J}=10-9$ K-ladder.

include a minimum of four detected transitions. The $\mathrm{CH}_{3} \mathrm{CCH}$ lines present a low velocity dispersion $\left(\leq 0.68 \mathrm{~km} \mathrm{~s}^{-1}\right)$ around the systemic velocity of the source $\left(\mathrm{V}_{\text {sys }}=-90 \mathrm{~km} \mathrm{~s}^{-1}\right)$, and a mean systemic velocity of $\bar{V}_{l s r}=-90.007 \pm 0.007 \mathrm{~km} \mathrm{~s}^{-1}$. Observed line widths also show an overall small dispersion, with FWHM values ranging from 4.15 to $5.68 \mathrm{~km} \mathrm{~s}^{-1}$. Moreover, line profiles are narrow, with average values of $4.94 \pm 0.77 \mathrm{~km} \mathrm{~s}^{-1}$. HervíasCaimapo et al. (2019) have observed several molecular lines toward G331 and classified them according to the line profile, with narrow lines tracing the emission from the core ambient medium and lines with broad wings tracing the outflow and shocked region (see \$1.3.1). Accordingly, we expect from the narrow line profiles that the observed $\mathrm{CH}_{3} \mathrm{CCH}$ emission is originated from a quiescent (not expanding) core medium.

The K-ladder spectral profile characteristic of symmetric tops is particularly advantageous because of the wide range of energies associated with the different levels contained 
Table 3.1 - Spectral lines of $\mathrm{CH}_{3} \mathrm{CCH}$ observed toward G331 and their parameters obtained from Gaussian fits.

\begin{tabular}{|c|c|c|c|c|c|c|c|c|c|}
\hline \multicolumn{4}{|c|}{ Quantum Numbers } & \multirow{2}{*}{$\begin{array}{c}\text { Frequency } \\
(\mathrm{MHz})\end{array}$} & \multirow{2}{*}{$\begin{array}{l}E_{u p} \\
(\mathrm{~K})\end{array}$} & \multirow{2}{*}{$\begin{array}{c}A_{i j} \\
\left(1 \times 10^{-5} \mathrm{~s}^{-1}\right)\end{array}$} & \multirow{2}{*}{$\begin{array}{c}\int T_{A} d v \\
\left(\mathrm{~K} \mathrm{~km} \mathrm{~s}^{-1}\right)\end{array}$} & \multirow{2}{*}{$\begin{array}{c}V_{l s r} \\
\left(\mathrm{~km} \mathrm{~s}^{-1}\right)\end{array}$} & \multirow{2}{*}{$\begin{array}{l}\text { FWHM } \\
\left(\mathrm{km} \mathrm{s}^{-1}\right)\end{array}$} \\
\hline $\mathrm{J}_{u}$ & $\mathrm{~K}_{u}$ & $-\mathrm{J}_{l}$ & $\mathrm{~K}_{l}$ & & & & & & \\
\hline 10 & 4 & 9 & 4 & 170853.576 & 160.7 & 1.43 & $0.23973 \pm 0.01$ & $-90.016 \pm 0.09$ & $4.382 \pm 0.187$ \\
\hline 10 & 3 & 9 & 3 & 170876.41 & 110.1 & 1.55 & $1.4306 \pm 0.015$ & $-89.886 \pm 0.024$ & $4.571 \pm 0.057$ \\
\hline 10 & 2 & 9 & 2 & 170892.726 & 74 & 1.63 & $1.7501 \pm 0.022$ & $-89.775 \pm 0.027$ & $4.415 \pm 0.064$ \\
\hline 10 & 1 & 9 & 1 & 170902.518 & 52.3 & 1.68 & $3.0455 \pm 0.062$ & $-89.739 \pm 0.044$ & $4.439 \pm 0.115$ \\
\hline 10 & 0 & 9 & 0 & 170905.783 & 45.1 & 1.70 & $3.9471 \pm 0.04$ & $-89.341 \pm 0.024$ & $4.994 \pm 0.065$ \\
\hline 11 & 4 & 10 & 4 & 187936.23 & 169.7 & 1.97 & $0.38405 \pm 0.013$ & $-90.404 \pm 0.081$ & $4.763 \pm 0.195$ \\
\hline 11 & 3 & 10 & 3 & 187961.342 & 119.2 & 2.10 & $1.8727 \pm 0.019$ & $-89.965 \pm 0.023$ & $4.677 \pm 0.055$ \\
\hline 11 & 2 & 10 & 2 & 187979.286 & 83 & 2.20 & $2.0664 \pm 0.024$ & $-89.842 \pm 0.025$ & $4.451 \pm 0.058$ \\
\hline 11 & 1 & 10 & 1 & 187990.055 & 61.4 & 2.25 & $3.5301 \pm 0.071$ & $-89.835 \pm 0.044$ & $4.568 \pm 0.115$ \\
\hline 11 & 0 & 10 & 0 & 187993.645 & 54.1 & 2.27 & $4.4260 \pm 0.044$ & $-89.389 \pm 0.024$ & $5.06 \pm 0.064$ \\
\hline 12 & 4 & 11 & 4 & 205018.114 & 179.6 & 2.63 & $0.48566 \pm 0.015$ & $-89.886 \pm 0.074$ & $4.98 \pm 0.177$ \\
\hline 12 & 3 & 11 & 3 & 205045.501 & 129 & 2.77 & $1.9662 \pm 0.021$ & $-89.988 \pm 0.025$ & $4.805 \pm 0.059$ \\
\hline 12 & 2 & 11 & 2 & 205065.07 & 92.9 & 2.88 & $2.0374 \pm 0.021$ & $-89.856 \pm 0.023$ & $4.518 \pm 0.052$ \\
\hline 12 & 1 & 11 & 1 & 205076.816 & 71.2 & 2.94 & $3.5154 \pm 0.065$ & $-89.936 \pm 0.041$ & $4.632 \pm 0.105$ \\
\hline 12 & 0 & 11 & 0 & 205080.732 & 64 & 2.96 & $4.4571 \pm 0.049$ & $-89.43 \pm 0.027$ & $5.184 \pm 0.071$ \\
\hline 13 & 3 & 12 & 3 & 222128.815 & 139.7 & 3.57 & $1.9676 \pm 0.037$ & $-90.04 \pm 0.043$ & $4.551 \pm 0.096$ \\
\hline 13 & 2 & 12 & 2 & 222150.01 & 103.5 & 3.69 & $2.3272 \pm 0.031$ & $-90.068 \pm 0.031$ & $4.841 \pm 0.076$ \\
\hline 13 & 1 & 12 & 1 & 222162.73 & 81.9 & 3.75 & $3.7371 \pm 0.09$ & $-90.149 \pm 0.058$ & $5.019 \pm 0.152$ \\
\hline 13 & 0 & 12 & 0 & 222166.971 & 74.6 & 3.78 & $4.4326 \pm 0.062$ & $-89.522 \pm 0.035$ & $5.241 \pm 0.09$ \\
\hline 14 & 4 & 13 & 4 & 239179.281 & 201.7 & 4.34 & $0.44333 \pm 0.016$ & $-90.617 \pm 0.073$ & $4.154 \pm 0.177$ \\
\hline 14 & 3 & 13 & 3 & 239211.215 & 151.1 & 4.51 & $2.0168 \pm 0.027$ & $-90.153 \pm 0.03$ & $4.554 \pm 0.068$ \\
\hline 14 & 2 & 13 & 2 & 239234.034 & 115 & 4.63 & $2.0813 \pm 0.026$ & $-90.092 \pm 0.028$ & $4.512 \pm 0.065$ \\
\hline 14 & 1 & 13 & 1 & 239247.728 & 93.3 & 4.70 & $3.6931 \pm 0.074$ & $-90.295 \pm 0.049$ & $5.209 \pm 0.13$ \\
\hline 14 & 0 & 13 & 0 & 239252.294 & 86.1 & 4.73 & $4.2518 \pm 0.058$ & $-89.497 \pm 0.034$ & $5.337 \pm 0.09$ \\
\hline 15 & 3 & 14 & 3 & 256292.63 & 163.4 & 5.59 & $2.2953 \pm 0.057$ & $-90.14 \pm 0.058$ & $5.113 \pm 0.159$ \\
\hline 15 & 2 & 14 & 2 & 256317.071 & 127.3 & 5.72 & $2.1340 \pm 0.056$ & $-90.023 \pm 0.062$ & $4.907 \pm 0.153$ \\
\hline 15 & 1 & 14 & 1 & 256331.739 & 105.7 & 5.80 & $3.2272 \pm 0.065$ & $-90.145 \pm 0.045$ & $4.765 \pm 0.117$ \\
\hline 15 & 0 & 14 & 0 & 256336.629 & 98.4 & 5.83 & $3.9996 \pm 0.055$ & $-89.538 \pm 0.037$ & $5.426 \pm 0.09$ \\
\hline 16 & 4 & 15 & 4 & 273336.519 & 227.1 & 6.64 & $0.58055 \pm 0.019$ & $-90.296 \pm 0.08$ & $5.451 \pm 0.223$ \\
\hline 16 & 3 & 15 & 3 & 273372.99 & 176.6 & 6.84 & $2.0837 \pm 0.024$ & $-90.173 \pm 0.027$ & $4.913 \pm 0.067$ \\
\hline 16 & 2 & 15 & 2 & 273399.051 & 140.4 & 6.97 & $2.1294 \pm 0.025$ & $-90.157 \pm 0.027$ & $4.931 \pm 0.069$ \\
\hline 16 & 1 & 15 & 1 & 273414.692 & 118.8 & 7.06 & $3.4784 \pm 0.061$ & $-90.417 \pm 0.043$ & $5.529 \pm 0.118$ \\
\hline 16 & 0 & 15 & 0 & 273419.906 & 111.5 & 7.09 & $3.8163 \pm 0.021$ & $-89.664 \pm 0.025$ & $5.478 \pm 0.09$ \\
\hline 17 & 3 & 16 & 3 & 290452.224 & 190.5 & 8.25 & $2.3997 \pm 0.049$ & $-90.469 \pm 0.044$ & $5.426 \pm 0.142$ \\
\hline 17 & 2 & 16 & 2 & 290479.904 & 154.4 & 8.40 & $2.0727 \pm 0.033$ & $-90.275 \pm 0.033$ & $4.931 \pm 0.102$ \\
\hline 17 & 1 & 16 & 1 & 290496.516 & 132.7 & 8.49 & $3.5350 \pm 0.062$ & $-90.682 \pm 0.044$ & $5.679 \pm 0.122$ \\
\hline 17 & 0 & 16 & 0 & 290502.054 & 125.5 & 8.52 & $3.8137 \pm 0.054$ & $-89.882 \pm 0.036$ & $5.448 \pm 0.096$ \\
\hline 18 & 3 & 17 & 3 & 307530.263 & 205.3 & 9.84 & $1.8777 \pm 0.021$ & $-90.228 \pm 0.027$ & $4.899 \pm 0.065$ \\
\hline 18 & 2 & 17 & 2 & 307559.559 & 169.1 & 1.00 & $1.7264 \pm 0.02$ & $-90.227 \pm 0.028$ & $4.764 \pm 0.066$ \\
\hline 18 & 1 & 17 & 1 & 307577.141 & 147.5 & 1.01 & $2.9083 \pm 0.038$ & $-90.496 \pm 0.033$ & $5.394 \pm 0.084$ \\
\hline 18 & 0 & 17 & 0 & 307583.003 & 140.3 & 1.01 & $3.2710 \pm 0.052$ & $-89.758 \pm 0.042$ & $5.575 \pm 0.11$ \\
\hline $\mathrm{J}_{u}$ & $\mathrm{~K}_{u}$ & $-\mathrm{J}_{l}$ & $\mathrm{~K}_{l}$ & Frequency $(\mathrm{MHz})$ & $E_{u p}(\mathrm{~K})$ & $A_{i j}\left(1 \times 10^{-5} \mathrm{~s}^{-1}\right)$ & $\int T_{A} d v\left(\mathrm{~K} \mathrm{~km} \mathrm{~s}^{-1}\right)$ & $V_{l s r}\left(\mathrm{~km} \mathrm{~s}^{-1}\right)$ & FWHM $\left(\mathrm{km} \mathrm{s}^{-1}\right)$ \\
\hline
\end{tabular}

within each ladder (see Table 3.1). The energy distribution of those different levels plays an important role in the interpretation of the observed data and the analyses performed on them. In Figure 3.2, a plot of the Einstein A coefficient versus the energy of each observed $\mathrm{CH}_{3} \mathrm{CCH}$ rotational level is presented. The transitions are grouped according to their $\mathrm{K}$ transitions, in order to facilitate comparisons between different K-ladders. 


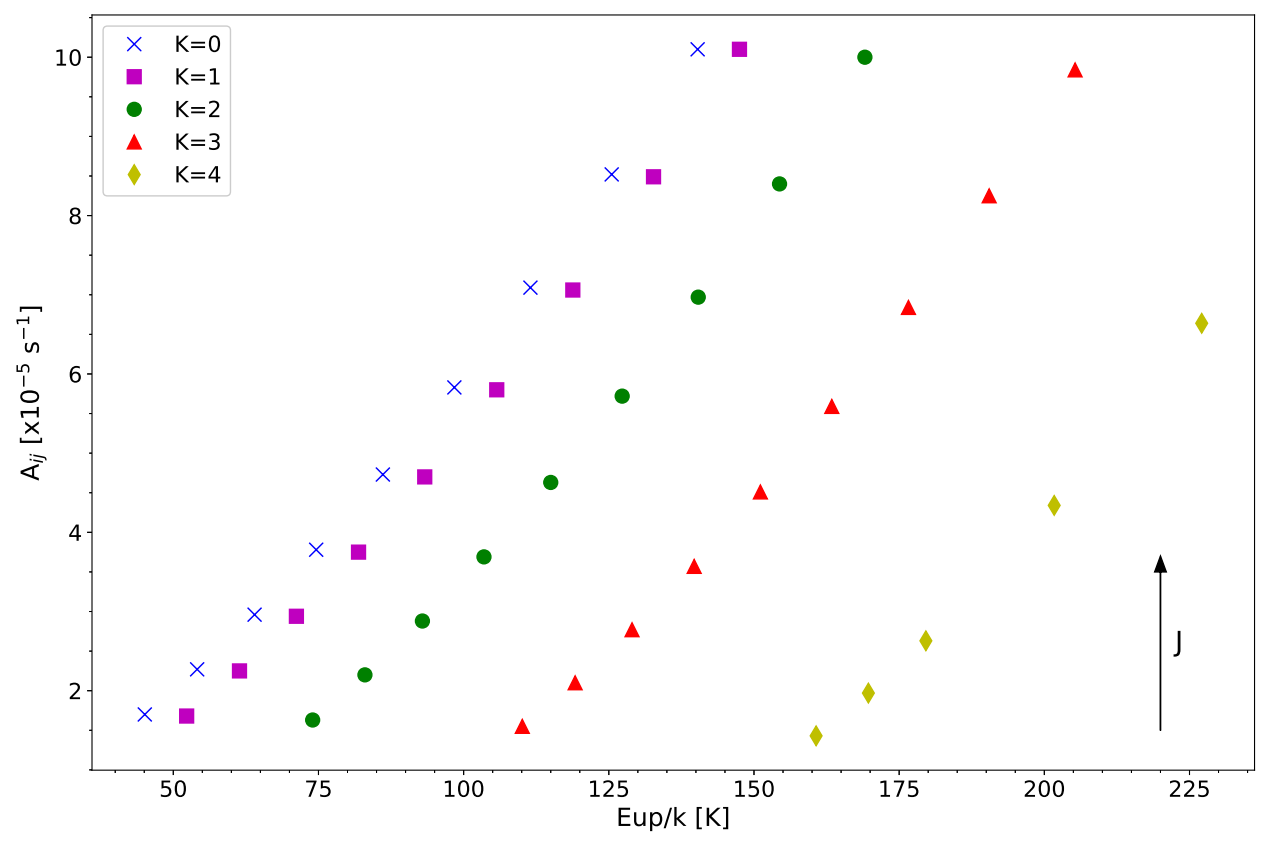

Figure 3.2: Plot of $\mathrm{A}_{i j}$ versus $\mathrm{E}_{u p}$ of the observed $\mathrm{CH}_{3} \mathrm{CCH}$ lines. The transitions are grouped according to their $\mathrm{K}$ quantum number: blue cross-marks correspond to transitions with $\mathrm{K}=0$, magenta squares correspond to $\mathrm{K}=1$, green circles correspond to $\mathrm{K}=2$, red triangles correspond to $\mathrm{K}=3$ and yellow diamonds correspond to $\mathrm{K}=4$.

Levels within a given K-ladder have similar values of $\mathrm{A}_{i j}$, which increases gradually with J. Moreover, the energy of the upper level also increases with J. Thus, states with higher $\mathrm{J}$ quantum numbers require higher energies to be populated, and therefore tend to be associated with warmer regions than the lower J states. Within each K-ladder, transitions with higher $\mathrm{K}$ quantum numbers present higher values of $\mathrm{E}_{u p}$, which results in the ladderlike spectral profile observed at lower temperatures (for a more detailed discussion see $\$ 3.3)$.

\subsection{LTE analyses}

\subsubsection{Rotational diagrams}

In order to estimate the excitation temperature $\left(\mathrm{T}_{\text {exc }}\right)$ and column density $\left(\mathrm{N}_{t o t}\right)$ of $\mathrm{CH}_{3} \mathrm{CCH}$ in $\mathrm{G} 331$, rotational diagrams were constructed. For a preliminary analysis, we adopted an average source size of $5^{\prime \prime}$, in accordance with previous observations of G331 (Mendoza et al., 2018; Duronea et al., 2019). The rotational diagram of $\mathrm{CH}_{3} \mathrm{CCH}$ 
constructed under the assumption of optically thin lines is displayed in blue in the upper panel of Figure 3.3. We derived $\mathrm{N}_{\text {tot }}\left(\mathrm{CH}_{3} \mathrm{CCH}\right)=(2.4 \pm 0.2) \times 10^{16} \mathrm{~cm}^{-2}$ and $\mathrm{T}_{\text {exc }}=47.5 \pm 1.4$ $\mathrm{K}$ from the fit $\left(\chi_{\text {red }}^{2}=2.95\right)$.
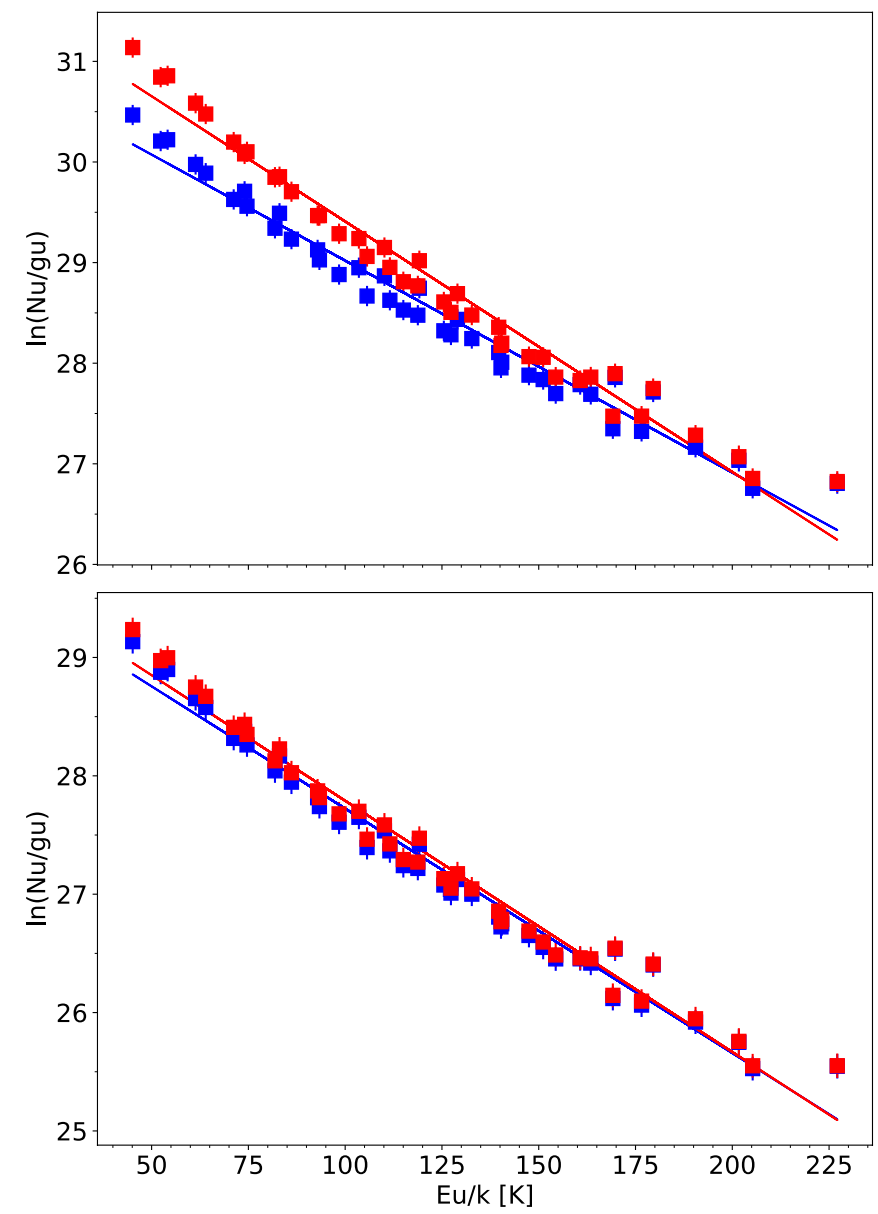

Figure 3.3: Upper panel: Rotational diagram of the $\mathrm{CH}_{3} \mathrm{CCH}$ lines for a source size of $5^{\prime \prime}$. Least squares fits with and without opacity corrections are shown in red and blue, respectively. Lower panel: Same as the upper panel, but for a source size of $10^{\prime \prime}$.

To explore the validity of the optically thin assumption, we estimated the lines' optical depths $(\tau)$ using CASSIS. The opacity corrected rotational diagram of $\mathrm{CH}_{3} \mathrm{CCH}$ is displayed in red in the upper panel of Figure 3.3. From the fit $\left(\chi_{r e d}^{2}=3.61\right)$ we obtained $\mathrm{N}_{\text {tot }}\left(\mathrm{CH}_{3} \mathrm{CCH}\right)=(3.9 \pm 0.3) \times 10^{16} \mathrm{~cm}^{-2}$ and $\mathrm{T}_{e x c}=40.2 \pm 1.1 \mathrm{~K}$. This correction leads to a new scaling of the diagram's ordinate axis, resulting in a decrease of $15 \%$ and an increase of $62 \%$ in the values of $\mathrm{T}_{\text {exc }}$ and $\mathrm{N}_{t o t}$, respectively.

Given the rather low temperatures derived from the rotational diagrams $\left(\mathrm{T}_{\text {exc }} \sim 40 \mathrm{~K}\right)$, the hypothesis of a compact emission arising from a region of $5^{\prime \prime}$ is unlikely. Rather, we infer that the $\mathrm{CH}_{3} \mathrm{CCH}$ emission is originated from a cooler gas in the outer envelope of 
G331, in accordance with other observational works toward star-forming regions (Andron et al. 2018; Hervías-Caimapo et al., 2019; Nagy et al., 2015, Churchwell and Hollis, 1983). Thus, we expect $\mathrm{CH}_{3} \mathrm{CCH}$ to trace a more extended region, ranging between $5^{\prime \prime}$ and $10^{\prime \prime}$. The rotational diagram constructed for a source size of $10^{\prime \prime}$ is displayed in the lower panel of Figure 3.3. From the fit under the optically thin assumption $\left(\chi_{r e d}^{2}=2.33\right)$, we derived $\mathrm{N}_{\text {tot }}\left(\mathrm{CH}_{3} \mathrm{CCH}\right)=(6.4 \pm 0.4) \times 10^{15} \mathrm{~cm}^{-2}$ and $\mathrm{T}_{e x c}=48.4 \pm 1.3 \mathrm{~K}$. After including the opacity correction factor, we derived $\mathrm{N}_{\text {tot }}\left(\mathrm{CH}_{3} \mathrm{CCH}\right)=(6.9 \pm 0.5) \times 10^{15} \mathrm{~cm}^{-2}$ and $\mathrm{T}_{\text {exc }}=47.1 \pm 1.3 \mathrm{~K}$ from the fit $\left(\chi_{r e d}^{2}=2.32\right)$. For this source size, the contribution of $\mathrm{C}_{\tau}$ to the diagram is much less significant than for a more compact emission. Indeed, the corrected fit yielded $\mathrm{T}_{e x c}$ and $\mathrm{N}_{t o t}$ values only slightly different from the optically thin scenario, with respective changes of $-2.68 \%$ and $7.24 \%$. Table 3.2 summarizes the excitation temperatures and column densities derived from the rotational-diagram analysis.

Table 3.2 - Values of $\mathrm{T}_{\text {exc }}$ and $\mathrm{N}_{\text {tot }}$ of $\mathrm{CH}_{3} \mathrm{CCH}$ toward G331 obtained from the analysis of the rotational diagrams.

\begin{tabular}{ccccc}
\hline \hline \multirow{2}{*}{ Parameter } & \multicolumn{2}{c}{ Source size: $5^{\prime \prime}$} & \multicolumn{2}{c}{ Source size: 10" } \\
& With C $_{\tau}$ & ${\text { Without } \mathrm{C}_{\tau}}^{\prime \prime}$ & With $_{\tau}$ & Without $_{\tau}$ \\
\hline$T_{\text {exc }}[\mathrm{K}]$ & $40.2 \pm 1.1$ & $47.5 \pm 1.4$ & $47.1 \pm 1.2$ & $48.4 \pm 1.3$ \\
$N\left[\times 10^{16} \mathrm{~cm}^{-2}\right]$ & $3.9 \pm 0.3$ & $2.4 \pm 0.2$ & $0.69 \pm 0.05$ & $0.64 \pm 0.04$ \\
$\chi_{\text {red }}^{2}$ & 3.61 & 2.95 & 2.32 & 2.33 \\
\hline
\end{tabular}

In terms of the reduced $\chi^{2}$ values, the fit of the $5^{\prime \prime}$ diagram with opacity corrections was less accurate in comparison with the uncorrected counterpart, whereas no significant change was observed for the $10^{\prime \prime}$ diagram. This could potentially be due to an overestimation of the lines' optical depths in the compact-emission scenario, in particular for the $\mathrm{K}=0$ and $\mathrm{K}=1$ transitions at lower $\mathrm{J}$ values. Indeed, the $\mathrm{CH}_{3} \mathrm{CCH}$ emission seems to be optically thin (Churchwell and Hollis, 1983; Fontani et al., 2002), especially for the range of J quantum numbers observed in this work. Thus, the underestimation of the source size is likely being compensated by an overestimation of the opacity — which explains why the opacity correction is almost negligible in the extended-emission scenario. In Figure 3.4, the estimated $\tau$ s assuming a source size of $10^{\prime \prime}$ are plotted against the line's upper energy. The more intense lines within a K-ladder, corresponding to $\mathrm{K}$ values of 0 and 1 , are associated with higher $\tau \mathrm{s}$, in comparison with the more optically thin $\mathrm{K} \geq 2$ lines. As $\mathrm{E}_{u p}$ increases, the optical depth of the $K=0,1$ transitions show a steep decrease. Moreover, optical depths also decrease with J. Given that $\tau \lesssim 0.2$, it is fair to assume an optically thin emission for 


\section{$\mathrm{CH}_{3} \mathrm{CCH}$.}

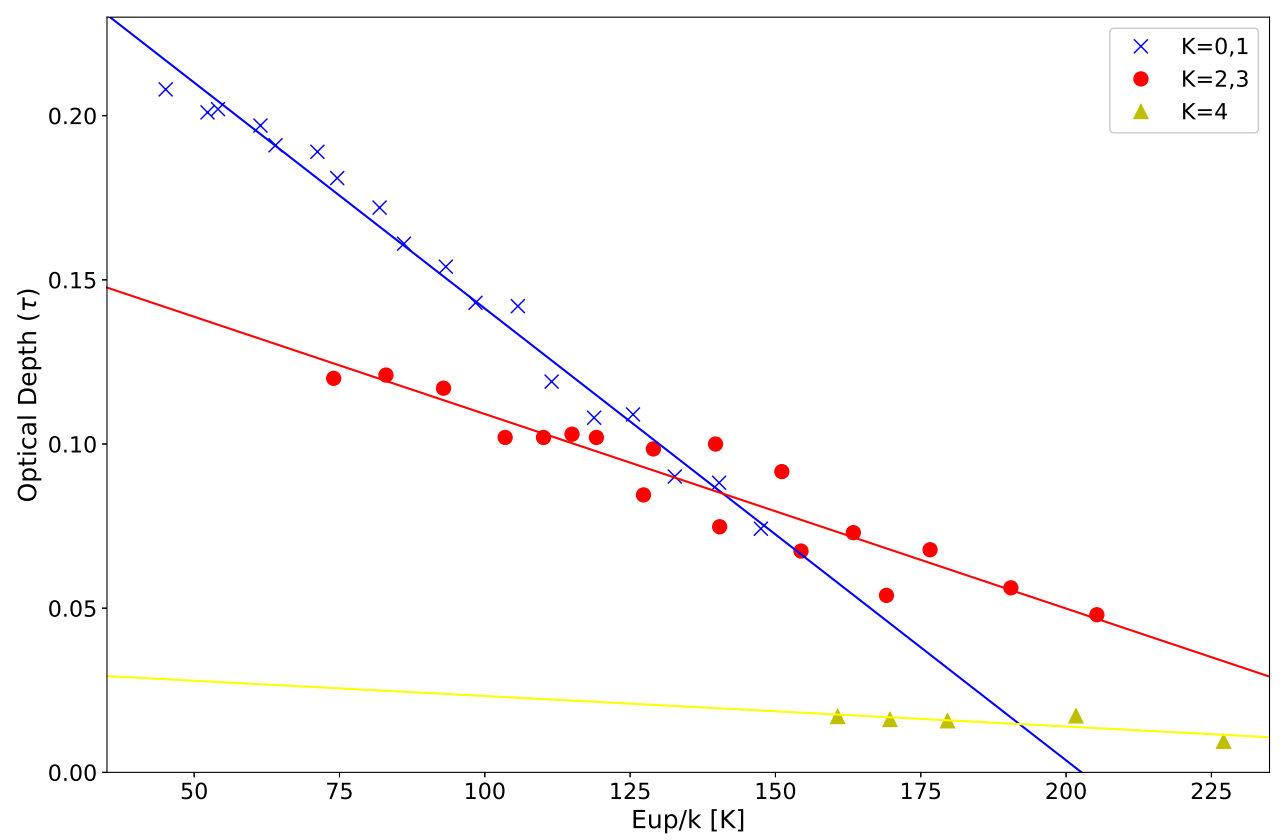

Figure 3.4: Optical depth $(\tau)$ vs. $\mathrm{E}_{u}$ of the $\mathrm{CH}_{3} \mathrm{CCH}$ lines assuming a source size of $10^{\prime \prime}$. Blue cross marks represent transitions with $K=0$ and $K=1$, red circles represent transitions with $K=2$ and $K=3$, and yellow triangles represent transitions with $\mathrm{K}=4$. Linear fits to the points are shown for each group of $\mathrm{K}$ quantum numbers, to guide the eye.

In both optically-thin and optically-thick scenarios, the $10^{\prime \prime}$ emission yielded better fits than the compact counterpart, which further supports the hypothesis of an extended $\mathrm{CH}_{3} \mathrm{CCH}$ emission. Nevertheless, this analysis alone in only conjectural, as interferometry data would be necessary to properly determine the emission size.

\subsubsection{Synthetic spectra}

The values of $\mathrm{T}_{e x c}$ and $\mathrm{N}_{t o t}$ derived from the rotational diagrams were employed to model the emission of $\mathrm{CH}_{3} \mathrm{CCH}$ under LTE conditions. We have simulated the spectra considering both $5^{\prime \prime}$ and $10^{\prime \prime}$ scenarios, and including the opacity correction. The modeled and observed spectra of the $\mathrm{J}=10-9$ and $\mathrm{J}=18-17 \mathrm{~K}$-ladders are shown in Figure 3.5 . Overall, the synthetic spectra are in good agreement with the observations, although the accordance is sensibly higher for K-ladders with lower J quantum numbers. Notwithstanding, the opacities of the $K=0$ and $K=1$ transitions in the lower K-ladders are clearly 
overestimated in the scenario of a compact emission - in agreement with our previous discussion.
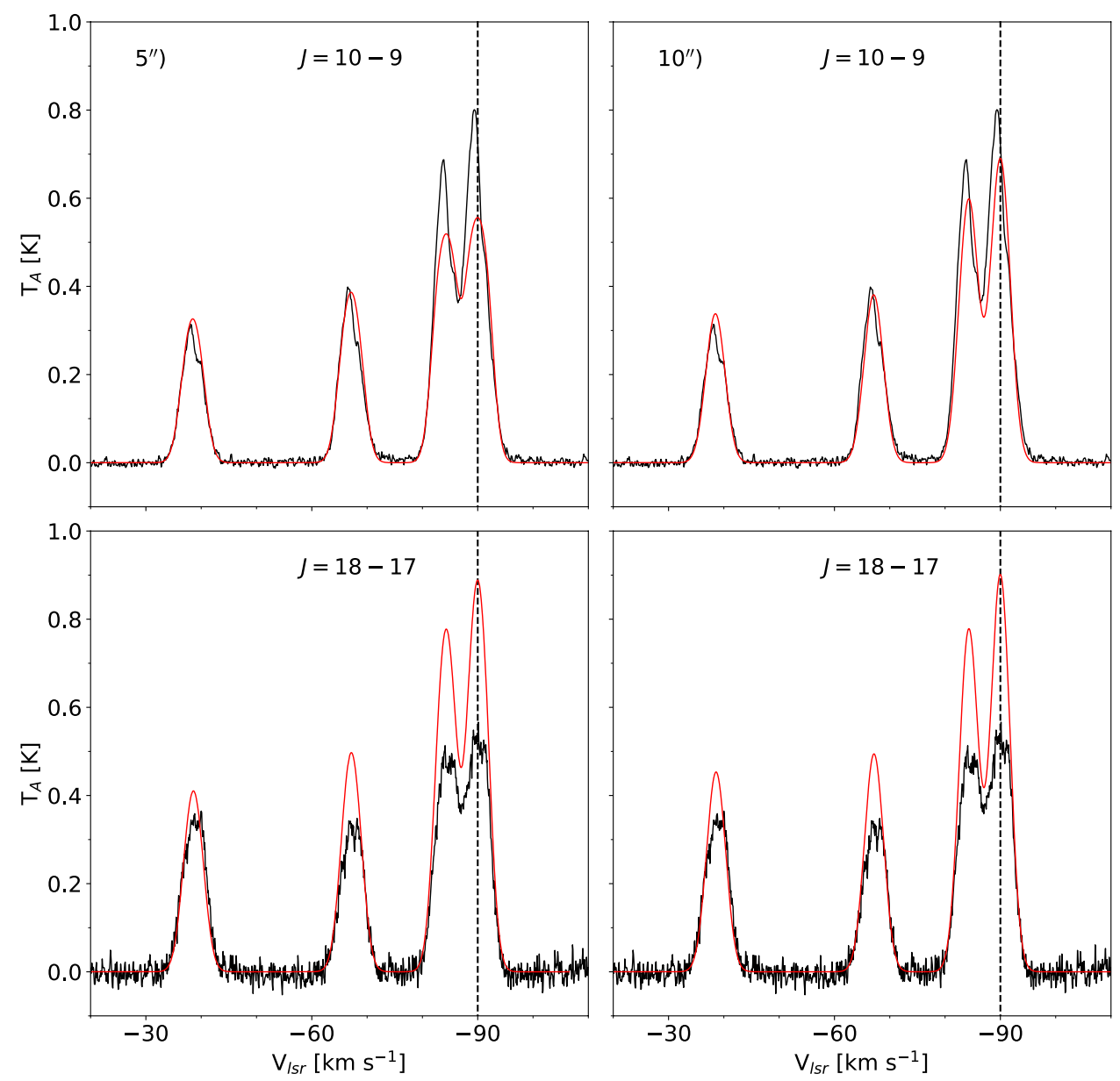

Figure 3.5: Spectra of $\mathrm{CH}_{3} \mathrm{CCH}$ observed toward G331. Upper pannels: J=10-9, 170.87-170.91 GHz; Lower pannels: J=18-17, 307.52-307.59 GHz. Left pannels: LTE models simulated for a compact emission $\left(5^{\prime \prime}\right)$. Right pannels: LTE models simulated for an extended emission $\left(10^{\prime \prime}\right)$. For all panels, the black and red spectra correspond to the observation and the LTE model, respectively. The dashed line indicates the systemic velocity of the source. The abscissas of all spectra were adjusted to the systemic velocity of the $\mathrm{K}=0$ transition.

The gradual decrease in accuracy of the models for higher K-ladders raises suspicion with respect to the LTE assumption. Moreover, the relative intensities of the $\mathrm{K}=2$ and $\mathrm{K}=3$ transitions predicted by the LTE models do not agree with the observations for all frequencies. Although the $\mathrm{K}=2$ lines are predicted to be more intense than the $\mathrm{K}=3$ even at higher frequencies, they are, in fact, observed to be less. Since the population distribution within each K-ladder is dictated by collisions (see \$1.7), this behavior implies, ipso facto, that the data cannot be described by only one temperature component. This supposition will be addressed further in $\$ 3.3$. Nonetheless, the synthetic spectra do accurately 
predict all of the observed $\mathrm{CH}_{3} \mathrm{CCH}$ lines to a reasonable extent. In Figure 3.6 we show a superposition of all observed and modeled K-ladders, assuming a source size of $10^{\prime \prime}$ and including opacity corrections. Thus, the $\mathrm{CH}_{3} \mathrm{CCH}$ line identifications are thereby definitely confirmed.
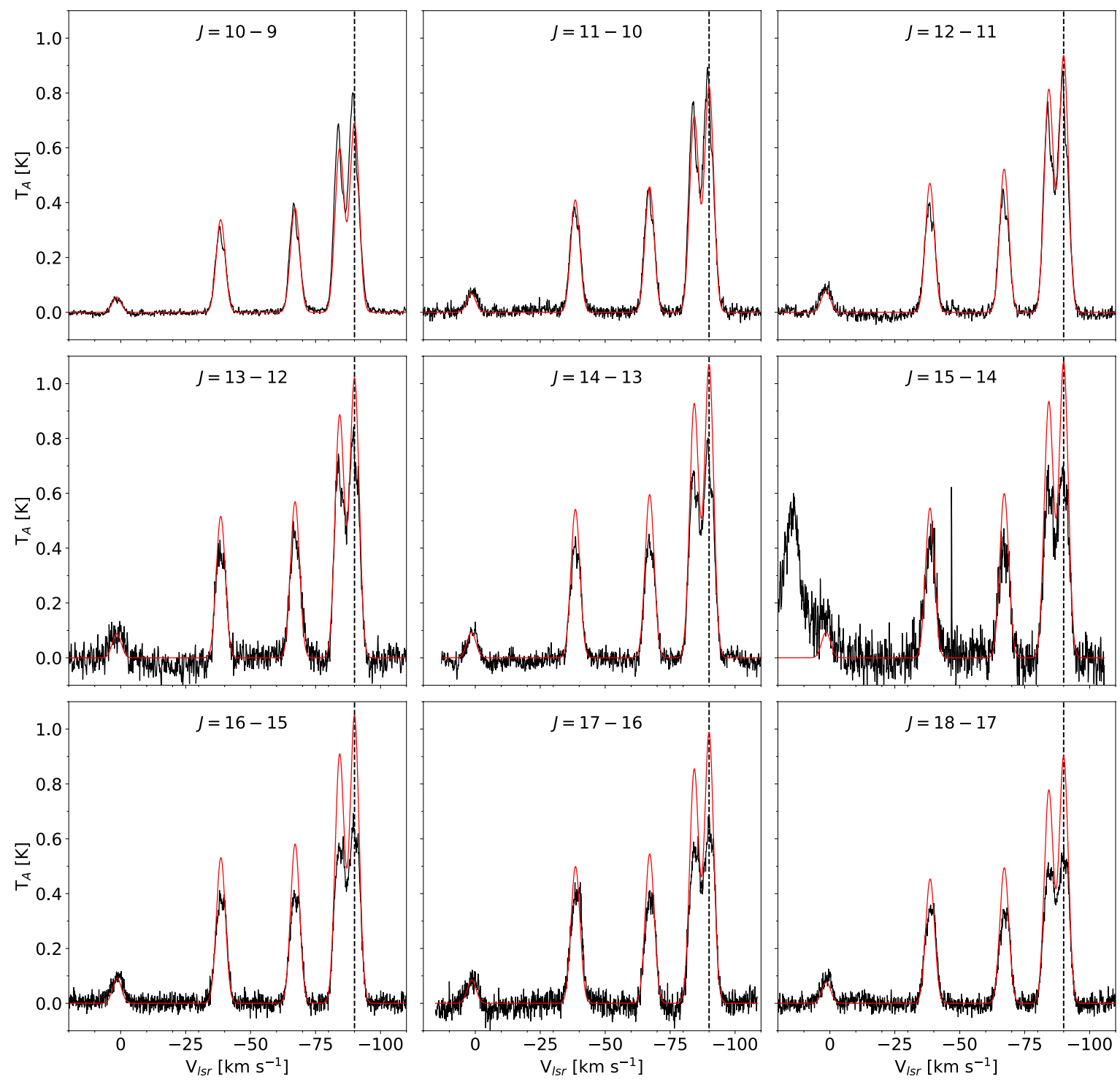

Figure 3.6: Observed spectra of the detected $\mathrm{CH}_{3} \mathrm{CCH}$ lines (black), superimposed by the LTE models (red). The dashed line indicates the systemic velocity of the source. The abscissas of all spectra were adjusted to the systemic velocity of the $\mathrm{K}=0$ transition.

\subsection{3 $\mathrm{CH}_{3} \mathrm{CCH}$ abundance}

From its column density, it is possible to infer the abundance of $\mathrm{CH}_{3} \mathrm{CCH}$ with respect to $\mathrm{H}_{2}$. This measure can then be compared to other environments, giving information on 
the chemical inventory and distribution of the ISM. The abundance of $\mathrm{CH}_{3} \mathrm{CCH}$ relative to $\mathrm{H}_{2}, \mathrm{X}\left(\mathrm{CH}_{3} \mathrm{CCH}\right)$, is defined as the ratio of the column densities $\mathrm{N}_{\text {tot }}\left(\mathrm{CH}_{3} \mathrm{CCH}\right) / \mathrm{N}_{\text {tot }}\left(\mathrm{H}_{2}\right)$. We have estimated the $\mathrm{H}_{2}$ column density in G331 from the ALMA measurements of $\mathrm{N}_{\text {tot }}\left(\mathrm{H}^{13} \mathrm{CO}^{+}\right) \approx(1.5-3) \times 10^{13} \mathrm{~cm}^{-2}$ (Merello et al., 2013a b; Hervías-Caimapo et al., 2019), adopting an abundance ratio $\mathrm{N}_{t o t}\left(\mathrm{H}^{13} \mathrm{CO}^{+}\right) / \mathrm{N}_{\text {tot }}\left(\mathrm{H}_{2}\right)=3.3 \times 10^{-11}$, as measured by Blake et al. (1987) in Orion KL. Then, by dividing the estimated $\mathrm{CH}_{3} \mathrm{CCH}$ column density (in the extended-emission and optically-corrected scenarios) by the inferred $\mathrm{N}_{t o t}\left(\mathrm{H}_{2}\right)$, we obtained a ratio of $\mathrm{X}\left(\mathrm{CH}_{3} \mathrm{CCH}\right) \approx(1.5-7.6) \times 10^{-8}$. Giannetti et al. (2017) have derived similar methyl acetylene abundances, $\mathrm{X}\left(\mathrm{CH}_{3} \mathrm{CCH}\right) \approx(0.5-2.8) \times 10^{-8}$, for a statistically significant sample of massive clumps with temperatures ranging from $\sim 20$ to $\sim 60 \mathrm{~K}$. This value is roughly one order of magnitude higher than the $\mathrm{CH}_{3} \mathrm{CCH}$ abundance in Sgr B2 (Churchwell and Hollis, 1983). For further comparison, Table 3.3 lists the $\mathrm{CH}_{3} \mathrm{CCH}$ abundances observed toward three massive star-forming regions (G12.89+0.49, G16.86-2.16 and G28.28-0.36) — in addition to G331 — as well as toward a low-mass star-forming core (L1527).

Table 3.3 - Abundances of $\mathrm{CH}_{3} \mathrm{CCH}$ observed toward high- and low-mass star-forming sites. ${ }^{a}$ From Taniguchi et al. (2019).

\begin{tabular}{cc}
\hline \hline Source & Abundance \\
\hline G12.89+0.49 & $2.1_{-1.1}^{+1.4} \times 10^{-8} a$ \\
G16.86-2.16 & $1.6_{-0.75}^{+0.95} \times 10^{-8} a$ \\
G28.28-0.36 & $3.8_{-2.2}^{+2.6} \times 10^{-8} a$ \\
L1527 & $(1.07 \pm 0.05) \times 10^{-9} a$ \\
G331 & $(1.5-7.6) \times 10^{-8}$ \\
\hline
\end{tabular}

Although the estimated abundance of $\mathrm{CH}_{3} \mathrm{CCH}$ shows good agreement with other observational works conducted toward similar sources, there is a caveat to our approach. The $\mathrm{N}_{t o t}\left(\mathrm{H}^{13} \mathrm{CO}^{+}\right)$column density derived for G331 was confined in a region of $\leq 5^{\prime \prime}$, which is much more compact than the expected $\mathrm{CH}_{3} \mathrm{CCH}$ emitting region. As a result, the inferred $\mathrm{N}_{\text {tot }}\left(\mathrm{H}_{2}\right)$ and $\mathrm{X}\left(\mathrm{CH}_{3} \mathrm{CCH}\right)$ are only approximations, with the former and the latter potentially being under- and overestimated, respectively. 


\subsection{Intensity inversion}

\subsubsection{Rotational-spectrum simulations}

The relative intensities of the lines within a given K-ladder are dictated by collisions, and thus will change depending on the temperature of the environment. For this reason, symmetric rotors act as excellent temperature probes, as was mentioned before. In particular, our observations of $\mathrm{CH}_{3} \mathrm{CCH}$ toward $\mathrm{G} 331$ show an unexpected behavior of the K-ladder profiles for different $\mathrm{J}$ transitions, which motivated us to explore this temperature dependence in a molecular level. Accordingly, we simulated the rotational spectra of $\mathrm{CH}_{3} \mathrm{CCH}$ at temperatures ranging from $10 \mathrm{~K}$ to $100 \mathrm{~K}$, with $10 \mathrm{~K}$ steps, by means of the PGOPHER general purpose software (Western, 2017). The rotational constants (A and B), the quartic and sextic centrifugal distortion constants $\left(\mathrm{D}_{J}, \mathrm{D}_{J K}, \mathrm{H}_{J}, \mathrm{H}_{J K}\right.$ and $\left.\mathrm{H}_{K J}\right)$, and the dipole moment $(\mu)$ employed in the simulations are listed in Table 3.4 .

Table 3.4 - Spectroscopic parameters employed in the spectrum simulations. The experimental measurements of the B rotational constant and the centrifugal distortion constants were obtained from Dubrulle et al. (1978). The dipole moment was measured by Muenter and Laurie (1966). Since the A rotational constant cannot be determined directly from the measured spectra, the estimated constant from the JPL database was used.

\begin{tabular}{cc|cc}
\hline \hline Parameter & Value & Parameter & Value \\
\hline A $[\mathrm{MHz}]$ & 158590 & $\mathrm{H}_{J}[\mathrm{~Hz}]$ & 0.0097 \\
B $[\mathrm{MHz}]$ & 8545.87712 & $\mathrm{H}_{J K}[\mathrm{~Hz}]$ & 0.935 \\
$\mathrm{D}_{J}[\mathrm{kHz}]$ & 2.9423 & $\mathrm{H}_{K J}[\mathrm{~Hz}]$ & 5.23 \\
$\mathrm{D}_{J K}[\mathrm{kHz}]$ & 163.423 & $\mu[\mathrm{D}]$ & 0.75 \\
\hline
\end{tabular}

The transitions' intensities are given by Equation 2.10, which is normalized to the partition function given in Equation 2.11. The rotational energies are calculated from Equation 2.9. For validation purposes, the $\mathrm{CH}_{3} \mathrm{CCH}$ rotational spectrum was also simulated at 300 $\mathrm{K}$, with $\mathrm{J}_{u}$ values up to 79 . In Figure 3.7, we present the spectrum at $300 \mathrm{~K}$ together with the experimental counterpart, measured at the same temperature. The predicted frequencies were very close to experiment, with an average error of $\sim 1.2 \mathrm{MHz}$ for $\mathrm{J}_{u} \leq 30$ and $\mathrm{K} \leq 4$, which corresponds to frequencies up to $\sim 500 \mathrm{GHz}$. The relative intensities of the transitions within each K-ladder also show a very good agreement between the simulated and experimental spectra (see the inset in Figure 3.7). Thus, this approach can be considered a reliable source of information on the temperature dependence of the K-ladder 
spectral profile.

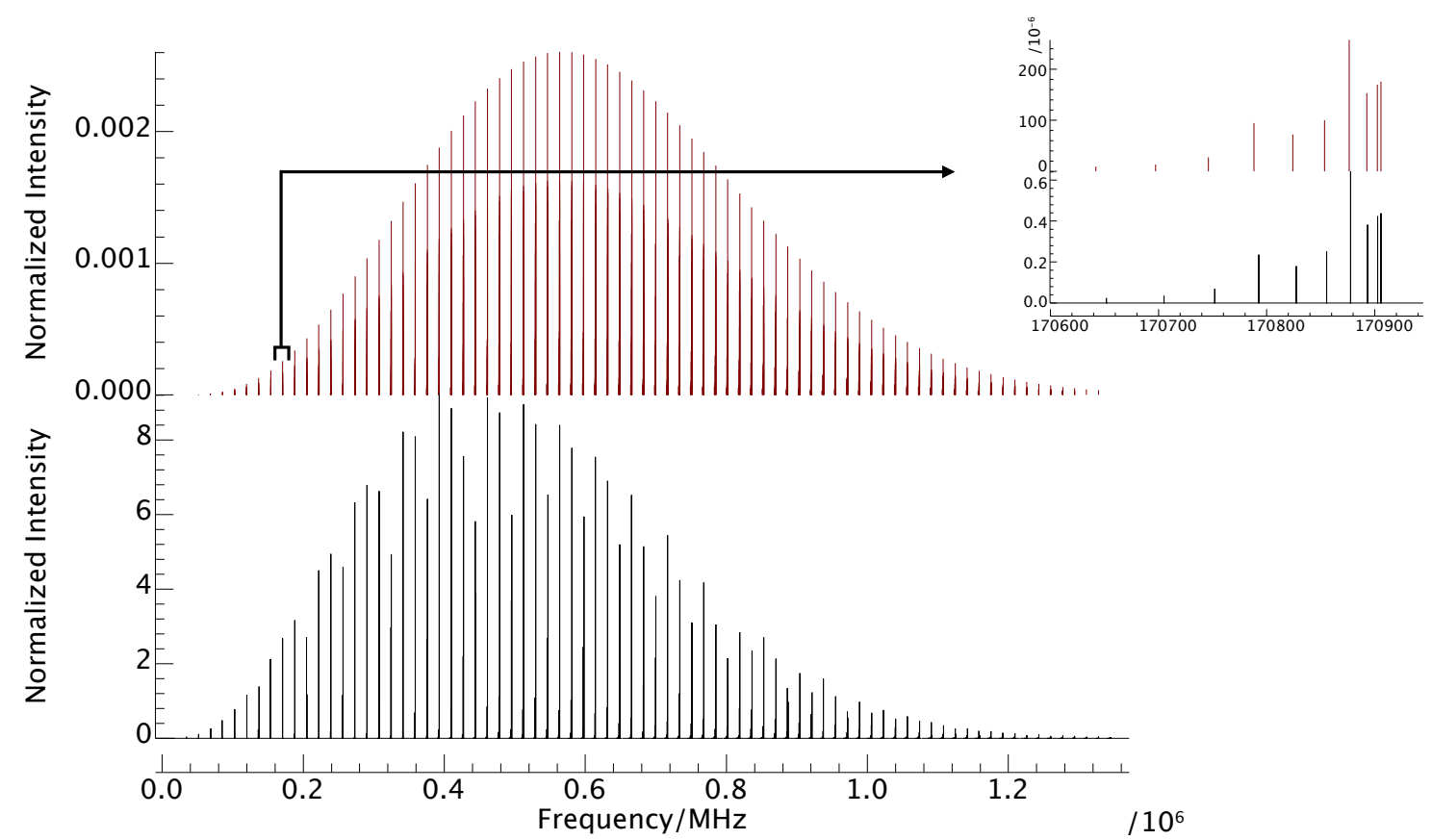

Figure 3.7: Experimental (red, upper panel) and simulated (black, lower panel) pure rotational spectra of $\mathrm{CH}_{3} \mathrm{CCH}$ at $300 \mathrm{~K}$. The inset shows the $\mathrm{J}=10-9 \mathrm{~K}$-ladder.

The $\mathrm{J}=10-9 \mathrm{~K}$-ladders of $\mathrm{CH}_{3} \mathrm{CCH}$ simulated at 10, 40, 70 and $100 \mathrm{~K}$ are displayed in Figure 3.8. Moreover, in Figure 3.9. we plot the intensity ratios $\mathrm{I}[\mathrm{K}=\mathrm{n}] / \mathrm{I}[\mathrm{K}=0]$ of the predicted lines within a K-ladder versus the temperature, with $n=1,2$ and 3 . We show the results for the $\mathrm{J}=10-9$ and $\mathrm{J}=18-17$ transitions, corresponding to the extremities of the observed bandwidth. As seen in the plot, the behavior of the intensity ratios are overall analogous for the range of $\mathrm{J}$ values explored in this work.

As speculated in the previous sections, an interesting phenomenon is evinced by Figures 3.8 and 3.9 . At low temperatures, the spectrum is dominated by the $\mathrm{K}=0$ transitions, with small contributions from the other $\mathrm{K}$ levels. As the temperature rises, however, the ratios of all $\mathrm{K}$ transitions increase at different rates. The intensity ratios of both $\mathrm{K}=1$ and $\mathrm{K}=2$ transitions, compared to $\mathrm{K}=0$, follow a similar trend: they show an initial stage of rapid growth, which becomes gradually slower as the temperature continues to rise. As can be seen for the $K=1$ transitions, the curve will eventually reach a plateau, which stabilizes the $\mathrm{K}=1 / \mathrm{K}=0$ intensity ratios at values around $0.8-0.9$. Comparatively, the ratios for $\mathrm{K}=3$ present a more dramatic increase. At around $50 \mathrm{~K}$, the $\mathrm{K}=3$ lines become more intense than the $\mathrm{K}=2$ transitions. Further, at around $100 \mathrm{~K}$, their intensities surpass those of the $\mathrm{K}=1$ transitions. At even higher temperatures, they ultimately become the most intense 

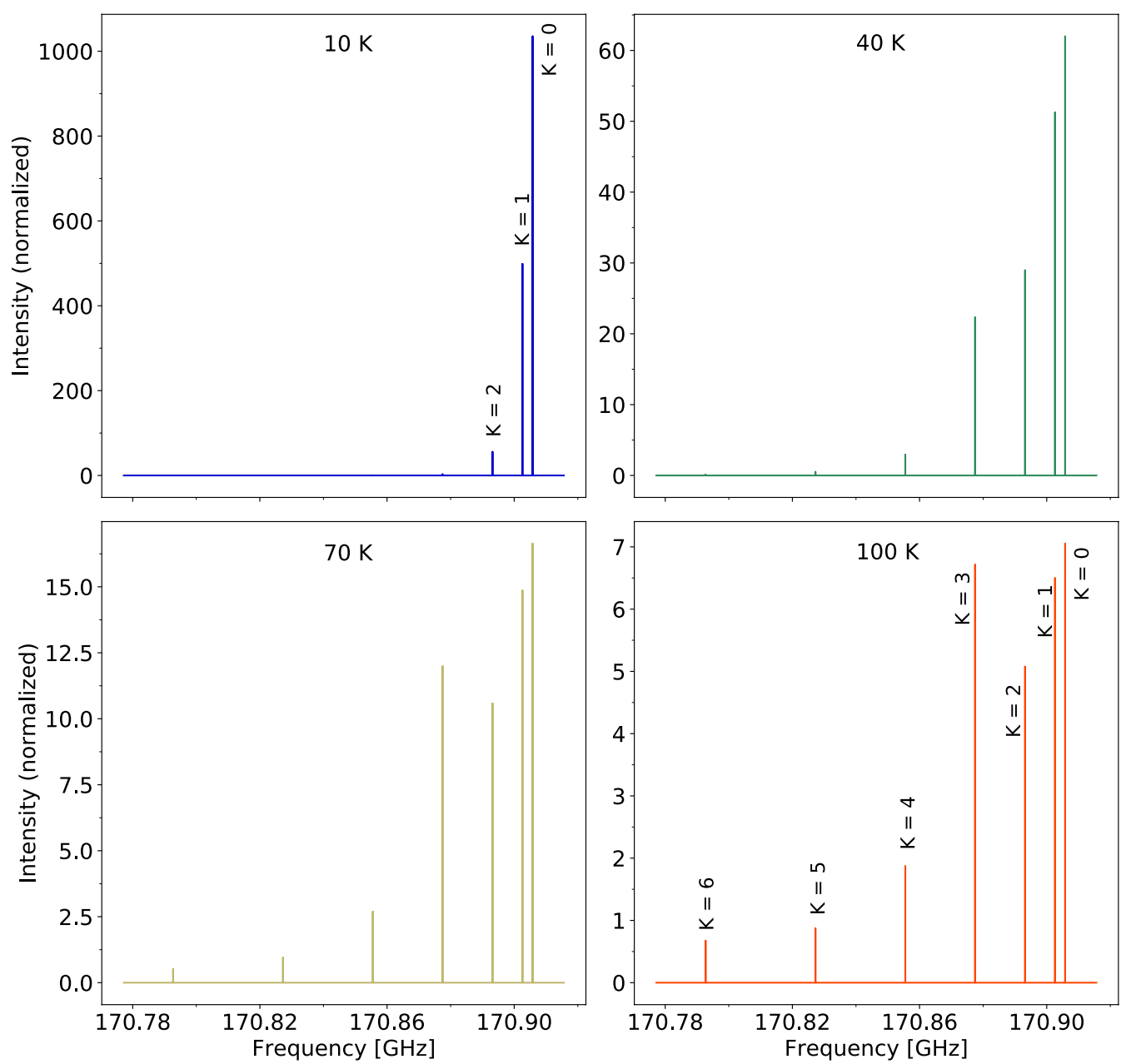

Figure 3.8: $\mathrm{J}=10_{K}-9_{K}$ K-ladder of $\mathrm{CH}_{3} \mathrm{CCH}$ simulated at $10 \mathrm{~K}$ (upper-left panel), $40 \mathrm{~K}$ (upper-right panel), $70 \mathrm{~K}$ (lower-left panel) and $100 \mathrm{~K}$ (lower-right panel).

line of the given K-ladder.

This behavior can be explained by nuclear spin statistics. Symmetric tops with three equivalent hydrogens, such as $\mathrm{CH}_{3} \mathrm{CCH}$ (see Figure 1.10), have two different spin symmetries - namely, A and E. The A states correlate with the transitions with $\mathrm{K}=0,3$, $6,9,12 \ldots$, whereas the E states correspond to the remaining transitions, and the states have relative statistical weights of $A: E=2: 1$ (Herzberg, 1945). As a result, the population distribution and therefore the relative intensities of the transitions at higher temperatures will favor the states with higher degeneracies, while, at lower temperatures, the energy acts as a limiting factor. For this reason, the $\mathrm{K}=3$ transition, which corresponds to a doubly-degenerate A state, rapidly increases in intensity as the temperature rises - eventually becoming the most intense line in a given K-ladder. This phenomenon is similar to the temperature-dependent abundance ratio of ortho/para hydrogen, and makes 


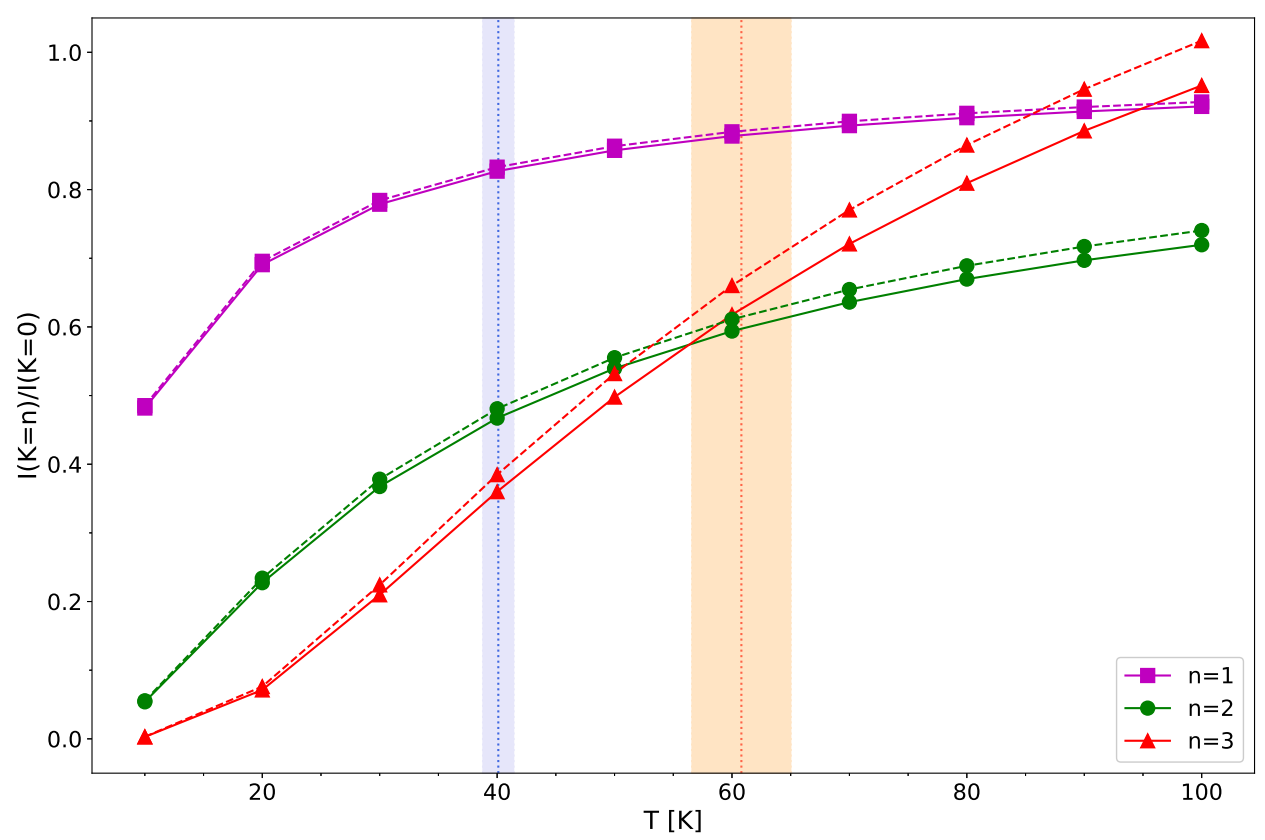

Figure 3.9: Intensity ratios $\mathrm{I}[\mathrm{K}=\mathrm{n}] / \mathrm{I}[\mathrm{K}=0]$ versus temperature for transitions within a given K-ladder. Purple squares represent ratios with $n=1$, green circles represent ratios with $n=2$, and red triangles represent ratios with $n=3$. Solid and dashed lines correspond to, respectively, the $\mathrm{J}=10-9$ and $\mathrm{J}=18-17$ K-ladders. The red and blue regions are discussed in 3.3 .4

the $\mathrm{CH}_{3} \mathrm{CCH}$ K-ladder profile highly sensitive to the local temperature, in particular with regards to the relative intensities of the $\mathrm{K}=3$ transitions.

\subsubsection{Inversion in the observed spectra}

As can be seen in Figure 3.1, we serendipitously observed a frequency window in which the $\mathrm{CH}_{3} \mathrm{CCH}$ K-ladder profiles gradually change as a function of the rotational quantum number. For low $\mathrm{J}$ values, which are associated with cooler regions, the $\mathrm{K}=2$ lines are more intense than the $\mathrm{K}=3$. However, as we observe transitions with higher $\mathrm{J}$ values, which are consequently associated with warmer regions, the $K=2 / K=3$ intensity ratios consistently decrease. From $\mathrm{J}=15-14$ onward, the $\mathrm{K}=3$ lines become more intense than the $\mathrm{K}=2$. This gradual inversion can be easily inferred by visual inspection, as is clearly shown in Figure 3.10 .

In order to quantify this inversion, we have plotted the $K=2 / K=3$ ratios of the observed areas listed in Table 3.1 as a function of the upper J value (Figure 3.11). To evaluate 


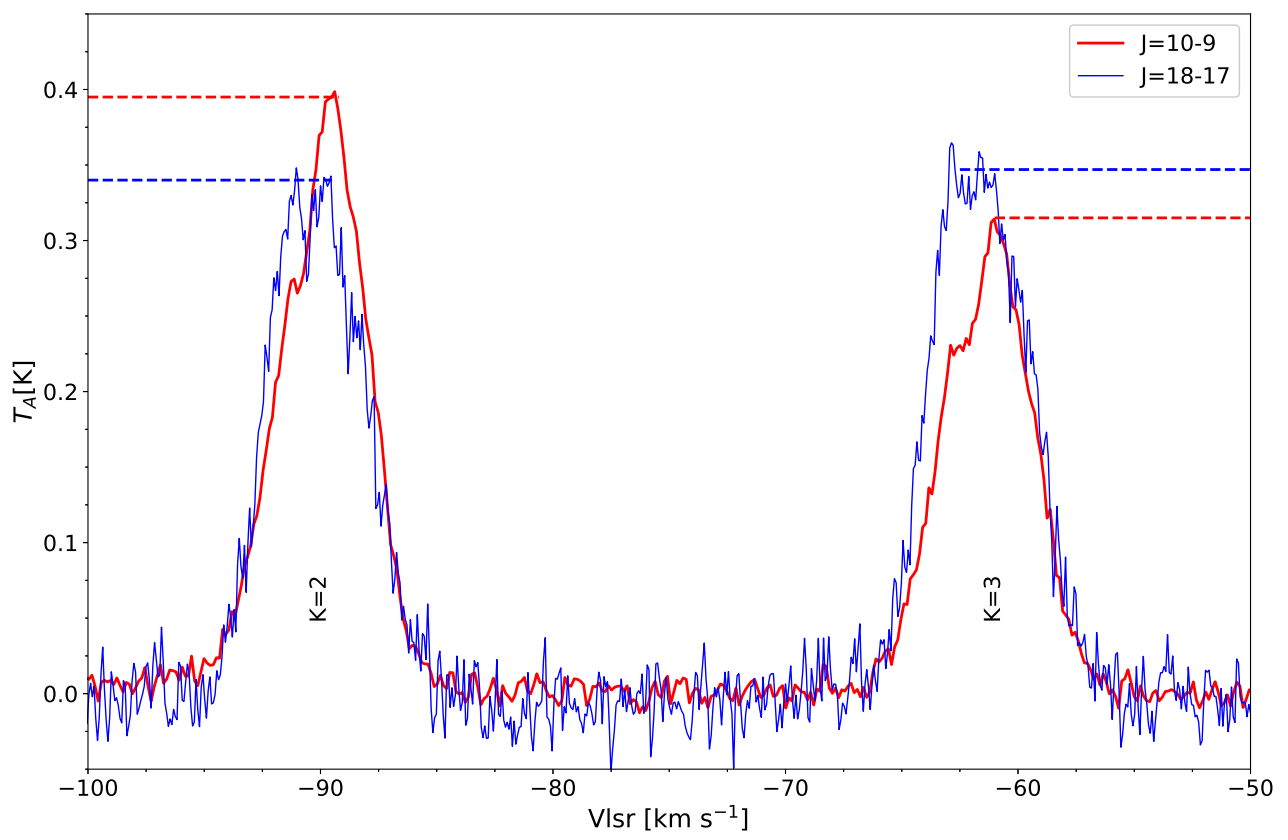

Figure 3.10: Superposition of the $\mathrm{K}=2$ and $\mathrm{K}=3$ lines of the $\mathrm{J}=10-9$ (red) and $\mathrm{K}=18-17$ (blue) K-ladders, evincing the intensity inversion of the peaks. The abscissa is adjusted to the systemic velocity of the $\mathrm{K}=2$ lines.

the strength of the correlation seen in the plot, we have derived its Pearson correlation coefficient $(r)$, which is calculated as the covariance of the two variables divided by the product of their standard deviation. Hence, this test calculates the normalized covariance of two variables, yielding a coefficient with values ranging from -1 to 1 . The -1 and 1 limits correspond, respectively, to perfectly-linear negative and positive correlations. The $r=0$ result corresponds to no correlation. A value of $r \geq|0.5|$ indicates a noticeable correlation, and $r \geq|0.8|$ is considered a strong correlation.

This plot of Figure 3.11 yields a Pearson correlation coefficient of $r=-0.84$, which, given the small FWHM dispersion of the observed lines, clearly indicates a decreasing trend of the $K=2 / K=3$ intensity ratios with $J$. This result strongly suggests that the lines are tracing a region with a temperature gradient, which explains the loss in accuracy of the synthetic LTE spectra for higher J transitions (see $\$ 3.2 .2$ ). 


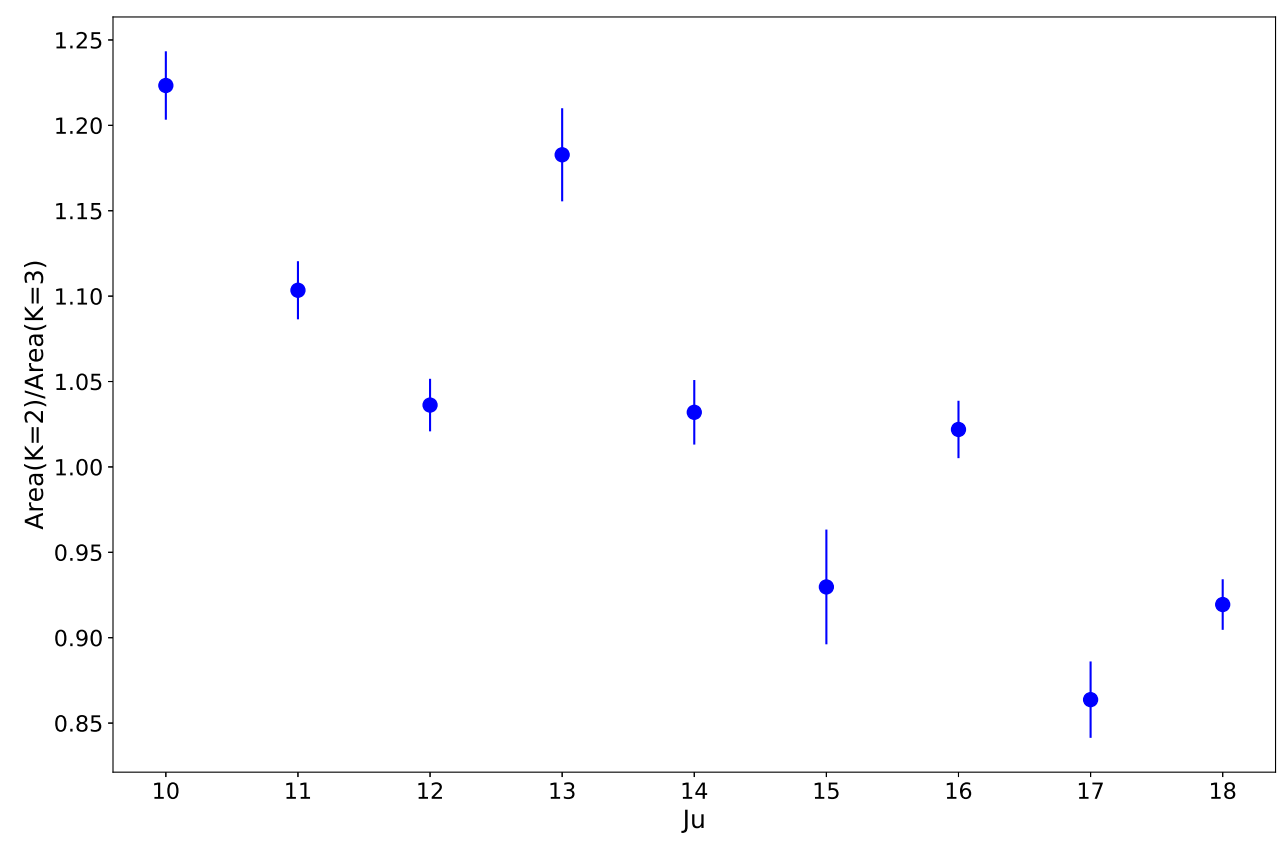

Figure 3.11: Ratios of the areas for $\mathrm{K}=2$ and $\mathrm{K}=3$ lines versus the upper $\mathrm{J}$ value of the transition. Areas and uncertainties were obtained through gaussian fittings of the observed data using CLASS.

\subsubsection{Modified rotational diagrams}

The rotational-diagram analysis described in $\$ 1.6$ works under the assumption of an isothermal gas in LTE. In such cases, there will be a linear correlation between $\ln \mathrm{N}_{u}$ and $\mathrm{E}_{u p}$. However, in a scenario where the lines cannot be characterized by only one temperature component, the rotational diagram will deviate from the linear trend. Mauersberger et al. (1988) have explored the case of a spherically symmetric cloud in which the temperature has a power-law dependence on the distance from the center, and demonstrated that, in those circumstances, there should be a linear correlation between $\ln \mathrm{N}_{u}$ and $\ln$ $\mathrm{E}_{u p}$. Fontani et al. (2002) have applied this reasoning to investigate the temperature profile toward 12 ultracompact HII regions. They constructed "modified" rotational diagramsnamely, ln $\mathrm{N}_{u}$ versus $\ln \mathrm{E}_{u p}$ - with observations of three $\mathrm{CH}_{3} \mathrm{CCH}$ K-ladders. Based on the quality of their linear fits, they concluded that the emission should originate from roughly isothermal regions.

To further investigate the hypothesis of a temperature gradient to describe the observed $\mathrm{CH}_{3} \mathrm{CCH}$ emission in G331, we have plotted modified rotational diagrams for both $5^{\prime \prime}$ and 
$10^{\prime \prime}$ source sizes. We also consider both optically thin and opacity corrected scenarios. The modified diagrams are shown in Figure 3.12. A comparison between the $\chi_{\text {red }}^{2}$ values of the linear fits of both the traditional and the modified rotational diagrams is presented in Table 3.5.

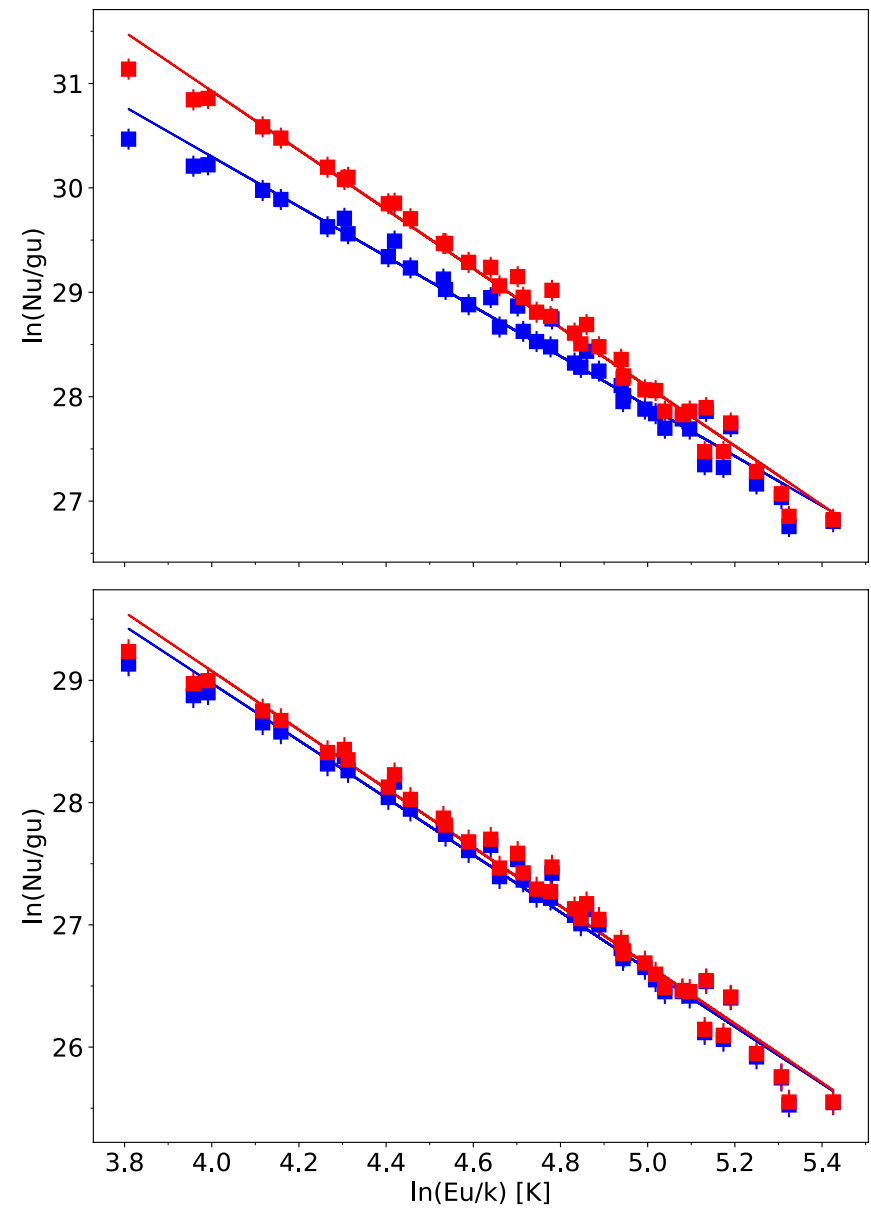

Figure 3.12: Upper panel: Modified rotational diagram of the $\mathrm{CH}_{3} \mathrm{CCH}$ lines for a source size of $5^{\prime \prime}$. Least squares fits with and without opacity corrections are shown in red and blue, respectively. Lower panel: Same as the upper panel, but assuming a source size of $10^{\prime \prime}$.

Table 3.5 - Values of $\chi_{\text {red }}^{2}$ obtained from the least square fits of the traditional and modified rotational diagrams.

\begin{tabular}{|c|c|c|c|}
\hline \multirow{2}{*}{$\begin{array}{c}\text { Source size } \\
\left({ }^{\prime \prime}\right)\end{array}$} & \multirow[t]{2}{*}{ Opacity correction } & \multicolumn{2}{|c|}{$\chi_{r e d}^{2}$} \\
\hline & & Traditional diagram & Modified diagram \\
\hline \multirow{2}{*}{5} & With $\mathrm{C}_{\tau}$ & 3.61 & 1.83 \\
\hline & Without $\mathrm{C}_{\tau}$ & 2.95 & 2.26 \\
\hline \multirow{2}{*}{10} & With $\mathrm{C}_{\tau}$ & 2.32 & 1.79 \\
\hline & Without $\mathrm{C}_{\tau}$ & 2.33 & 1.85 \\
\hline
\end{tabular}

For all the explored situations, the linear fits of the modified rotational diagrams were 
consistently better than the traditional counterparts, favoring the hypothesis of an emission with a non-negligible temperature gradient.

\subsubsection{Quantifying the temperature gradient}

At this point, the supposition of a temperature gradient is deemed fairly substantiated by the amount of evidence presented in the previous subsections. Thus, for the next step, we estimated the extent of the temperature profile by grouping the points in the rotational diagram into two subsets (Figure 3.13). The groups were separated at $\mathrm{E}_{u p} \approx 125 \mathrm{~K}$, according to the behavior of the observed $\mathrm{K}=2$ and $\mathrm{K}=3$ intensity ratios. The lower-energy group contains all $\mathrm{K}=2$ transitions with $\mathrm{J} \leq 14$, for which $\mathrm{K}=2 / \mathrm{K}=3 \geq 1$. Contrarily, the higher-energy subset encompasses all $K=2$ transitions with $J_{\iota} 14$, for which $K=2 / K=3{ }_{j} 1$. The resulting $\mathrm{T}_{\text {exc }}$ and $\mathrm{N}_{t o t}$ obtained from the linear fit of each subset are listed in Table 3.6.

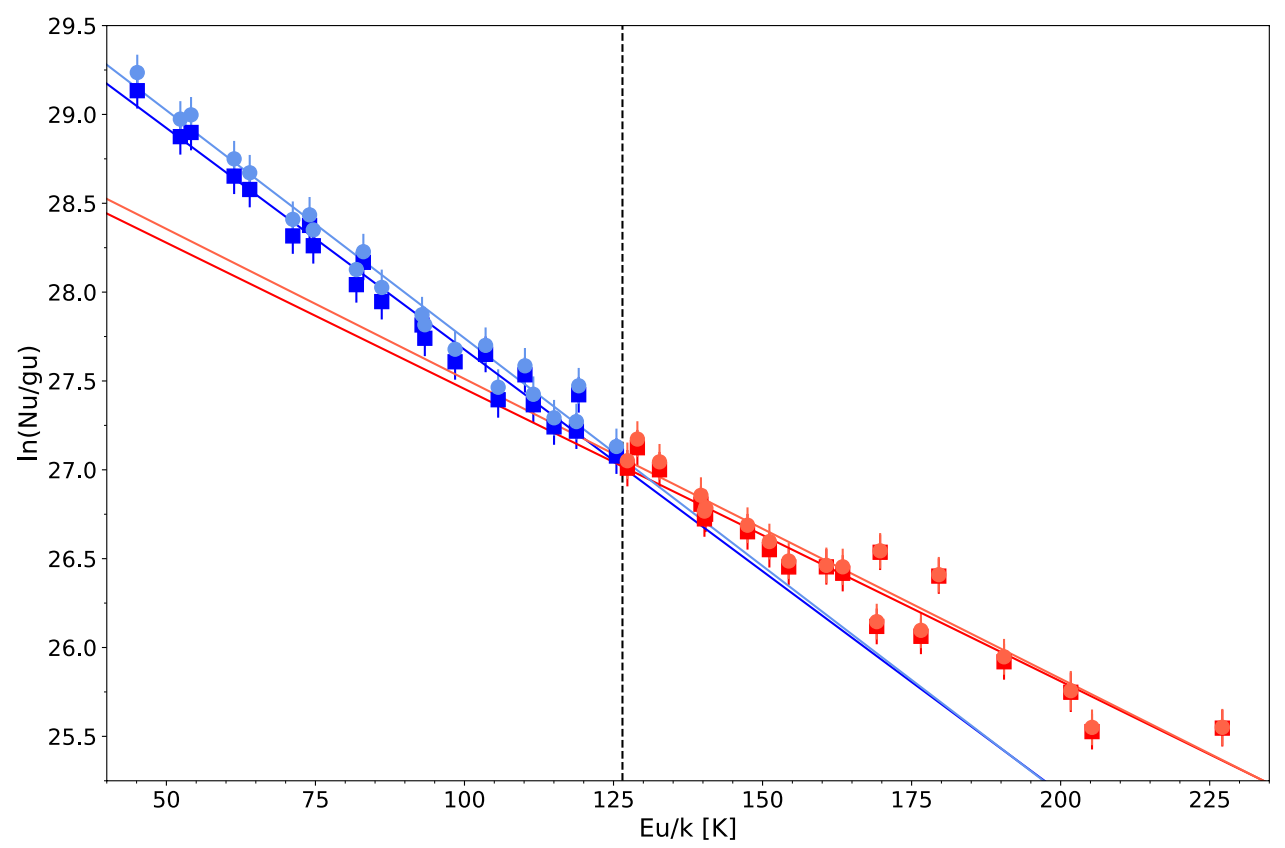

Figure 3.13: rotational diagram of the $\mathrm{CH}_{3} \mathrm{CCH}$ lines for a source size of $10^{\prime \prime}$. The points were divided into two subsets indicated by the dashed line. The points pertaining to the low- and high-energy subsets are shown in blue and red, respectively. Least squares fits to the points within each subset are shown. Circles and squares correspond to the lines with and without opacity corrections, respectively.

Assuming an optically thick scenario, the rotational diagram divided into two subsets yields temperature components of $\sim 40$ and $\sim 60 \mathrm{~K}$. Although the points in the rotational diagram show an overall fairly linear trend, the $\mathrm{T}_{\text {exc }}$ obtained from this analysis is highly 
Table 3.6 - Values of $\mathrm{T}_{\text {exc }}$ and $\mathrm{N}_{t o t}$ of $\mathrm{CH}_{3} \mathrm{CCH}$ obtained from the 2-component rotational diagrams for a source size of $10^{\prime \prime}$.

\begin{tabular}{ccccc}
\hline \hline Parameter & \multicolumn{2}{c}{ Lower-energy comp. (blue) } & \multicolumn{2}{c}{ Higher-energy comp. (red) } \\
& ${\text { With } \mathrm{C}_{\tau}}$ & Without $\mathrm{C}_{\tau}$ & With $\mathrm{C}_{\tau}$ & Without $\mathrm{C}_{\tau}$ \\
\hline$T_{\text {exc }}[\mathrm{K}]$ & $39.0 \pm 1.2$ & $40.1 \pm 1.3$ & $59.3 \pm 3.9$ & $60.8 \pm 4.2$ \\
$N\left[\times 10^{15} \mathrm{~cm}^{-2}\right]$ & $7.5 \pm 0.5$ & $6.9 \pm 0.5$ & $4.8 \pm 0.9$ & $4.5 \pm 0.8$ \\
$\chi_{\text {red }}^{2}$ & 0.72 & 0.78 & 1.62 & 1.74 \\
\hline
\end{tabular}

sensitive to the fitted slope. Consequently, a difference of around $20 \mathrm{~K}$ arises between the values derived from the linear fittings of the two subsets. This temperature interval coincides with the region of Figure 3.9 where the intensity inversion of $K=2$ and $K=3$ occurs. Thus, one can regard the two temperature components extracted from the blue and red subsets in Figure 3.13 as, respectively, lower and upper limits to the temperature profile of the observed emitting region - indicated as the blue and red regions in Figure 3.9. Not coincidentally, the rotational diagram analysis of \$3.2.1 yielded an averaged temperature of $T_{\text {exc }} \sim 50 \mathrm{~K}$, which corresponds approximately to the threshold where the intensity inversion of the $\mathrm{K}=2$ and $\mathrm{K}=3$ lines happens for the observed $\mathrm{J}$ transitions. The rotational diagram is an averaged approach, and therefore is suited to evaluate the largescale emission of $\mathrm{CH}_{3} \mathrm{CCH}$. The intrinsic spectroscopic properties of methyl acetylene, however, enable a more direct and meticulous assessment of the temperature of the source, giving information beyond the limitations of the rotational diagram. The same discussion is valid for the optically thin scenario.

The synthetic spectra under the LTE assumption shown in Figure 3.5 reproduces more accurately the observed data for lower J quantum numbers. This gradual loss in accuracy for higher $\mathrm{J}$ transitions is likely a consequence of the intensity inversion that occurs around $\mathrm{J}_{u}=14$ toward G331 (see Figure 3.11). Indeed, throughout the entire $\sim 170840$ $307600 \mathrm{MHz}$ frequency range, modeled transitions with $\mathrm{K}=3$ are less intense than the $\mathrm{K}=2$ counterparts, as shown in Figure 3.6. Since our observations cannot be accurately described by only one temperature component, the model will be increasingly amiss as the bulk of the emission transitions to regions with different average gas temperatures than the $\mathrm{T}_{\text {exc }}$ derived from the rotational diagram. Hence, our LTE model seems to best describe the cooler environment, traced by the lines with lower J values. Ergo, the rotational diagram is apparently biased toward the lower-temperature gas. 
Previously, Hervías-Caimapo et al. (2019) have used ALMA to observe four $\mathrm{CH}_{3} \mathrm{CCH}$ lines within the $\mathrm{J}=21-20 \mathrm{~K}$-ladder toward G331, which showed a peak of emission located at a radius of $\sim 1.2^{\prime \prime}$. From the rotational diagram constructed with the $\mathrm{CH}_{3} \mathrm{CCH}$ lines, they obtained an excitation temperature of $\mathrm{T}_{e x c}=70 \pm 7 \mathrm{~K}$. Their results are in accordance with our hypothesis of a temperature gradient, which indeed predicts that transitions with higher $\mathrm{J}$ quantum numbers will be associated with higher excitation temperatures and more compact regions.

\subsection{Kinematics}

\subsubsection{Velocity vs. frequency}

The transitions' spectroscopic parameters (see Table 3.1), obtained through fitting a Gaussian profile to each line, are directly related to the kinematics of the emitting regionor, at least, of the gas where the bulk of the emission is coming from. By examining the line shape variation as a function of the excitation energy, one can gather information on the small-scale structure of the source even with low-angular-resolution data. In Figure 3.14, we show plots of the peak velocities of the lines versus their frequencies. For the sake of clarity, each K transition was plotted separately. Since they are fainter and harder to observe, the $\mathrm{K}=4$ transitions were not included in this analysis.

In all of the plots, the lines' peak velocities are strongly negatively correlated with frequency, with Pearson coefficients of $r=-0.93,-0.94,-0.94$ and -0.88 for, respectively, $\mathrm{K}=0, \mathrm{~K}=1, \mathrm{~K}=2$ and $\mathrm{K}=3$. Moreover, the mean velocity shift between lines with the same $\mathrm{K}$ quantum number within the $\mathrm{J}=10-9$ and $\mathrm{J}=18-17 \mathrm{~K}$-ladders is $\sim 0.49 \mathrm{~km} \mathrm{~s}^{-1}$, which is almost twice the poorest obtained velocity resolution (see $\$ 2.2$ ). Those numbers bolster the claim that the $\mathrm{CH}_{3} \mathrm{CCH}$ lines with higher excitation energies, and therefore higher frequencies, are tracing regions with slightly different physical conditions. The lines associated with colder regions (i.e., with lower frequencies) are characterized by more redshifted velocities, whereas warmer regions seem to be systematically blueshifted.

\subsubsection{FWHM vs. frequency}

To inspect the line width as a function of the transition energy, we plotted the lines' FWHMs versus their rest frequencies (Figure 3.15). Again, different $\mathrm{K}$ transitions are 

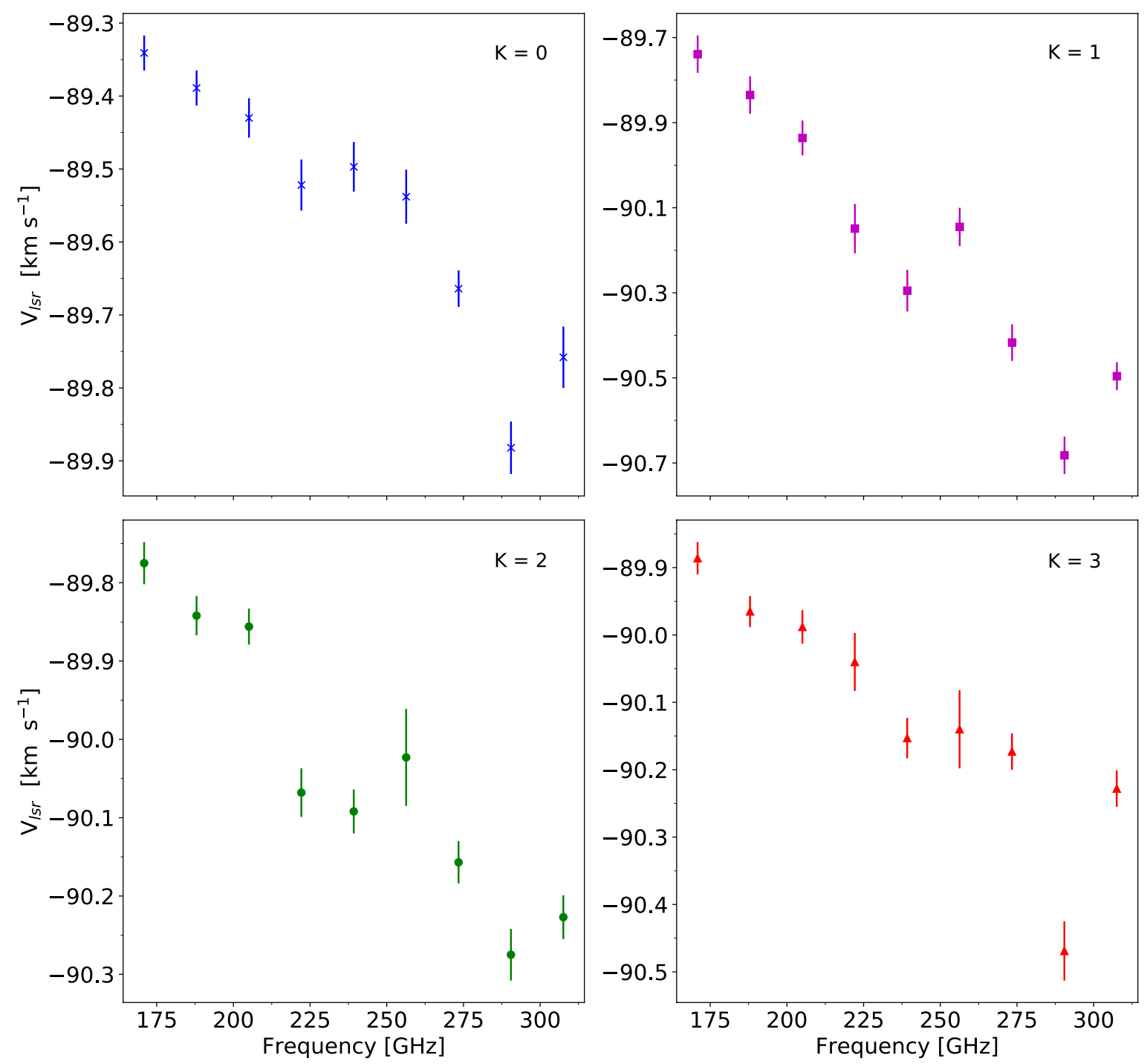

Figure 3.14: Peak velocities versus line frequency obtained from gaussian fittings to the $\mathrm{CH}_{3} \mathrm{CCH}$ lines. Each plot contains only transitions with the same K quantum number.

plotted separately, and the $K=4$ lines were not included in the analysis. From the plot, it is clear that the FWHM is correlated to the line frequency, with $r=0.98,0.88,0.77$ and 0.67 for $K=0, K=1, K=2$ and $K=3$. The $K=0$ lines show a particularly strong correlation, which presents a quasi-linear trend. This relationship implies that warmer regions are associated with broader lines.

The increase in FWHM for transitions with higher frequencies cannot be explained by thermal broadening, since its effects are negligible for radio lines. The contribution to the FWHM due to thermal broadening is calculated by (Draine, 2011):

$$
F W H M=2 \sqrt{\ln 2} b
$$



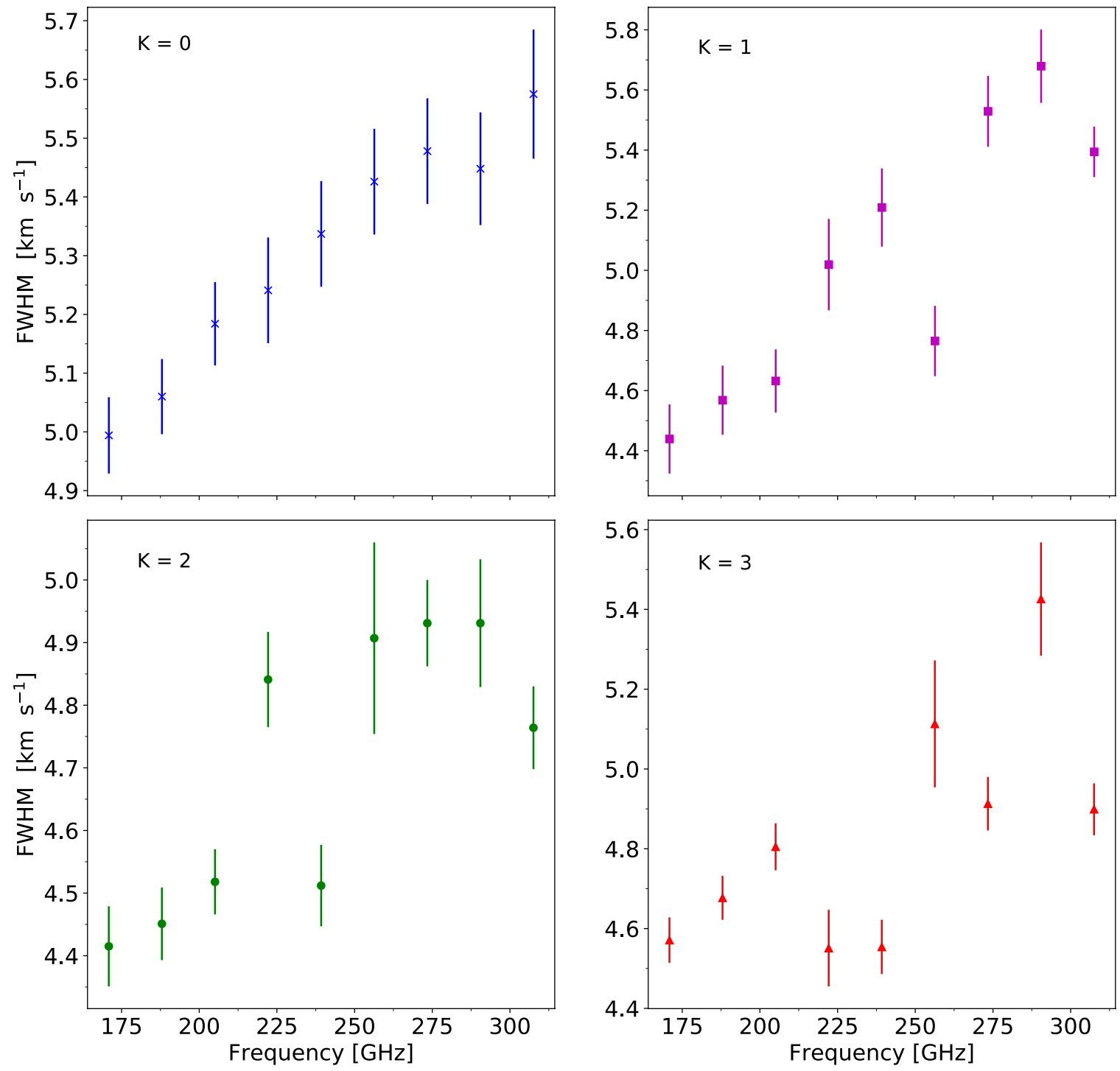

Figure 3.15: Line widths vs. rest frequencies obtained from gaussian fittings of the observed transitions. Each subplot contains only transitions with the same K quantum number

in which

$$
b=\sqrt{\frac{2 k T}{m}}
$$

As discussed in $\$ 3.3 .4$, the gas where the bulk of the $\mathrm{CH}_{3} \mathrm{CCH}$ emission originates is believed to present a temperature profile with lower and upper limits of, respectively, $\sim 40$ and $\sim 60 \mathrm{~K}$. At such temperatures, the contribution to the $\mathrm{CH}_{3} \mathrm{CCH}$ line width from thermal broadening is of $\sim 0.21$ and $\sim 0.26 \mathrm{~km} \mathrm{~s}^{-1}$, respectively, which corresponds to a variation of $\sim 1.25 \%$ of the line widths, assuming a FWHM of $\sim 4 \mathrm{~km} \mathrm{~s}^{-1}$. This contribution is ten times smaller than the average FWHM variation between the $\mathrm{J}=10-9$ and $\mathrm{J}=18-17$ 
lines with the same K quantum number.

Instead, the line broadening is likely to be caused by turbulence effects. In other words, the broader widths associated with higher $\mathrm{J}$ values indicate that the gas where the bulk of the emission arises from must become gradually more turbulent as the temperature increases. This is a fairly reasonable suggestion, considering that the warmer emission comes from the inner parts of the envelope surrounding the MYSO. A similar reasoning is discussed by Fontani et al. (2002), who observed lines of $\mathrm{CH}_{3} \mathrm{CCH}$ toward 12 UC HII regions and also found a positive correlation between their widths and frequencies.

\subsubsection{FWHM vs. velocity}

In the previous subsections, we demonstrated that both the line widths and peak velocities of the observed $\mathrm{CH}_{3} \mathrm{CCH}$ transitions correlate with their frequencies, giving information on the small-scale structure of G331. Now, we investigate the correlation between those two parameters. In Figure 3.16, we present a plot of the widths and peak velocities of the observed lines, which are listed in Table 3.1 .

The line widths and peak velocities of transitions with the same K quantum number are overall strongly correlated. Indeed, the Pearson correlation coefficient of the $K=0, K=1$, $\mathrm{K}=2$ and $\mathrm{K}=3$ groups are, respectively, $r=-0.85, r=-0.97, r=-0.78$, and $r=-0.80$. As for the previous subsections, the lines with $\mathrm{K}=4$ were not included in the comparison. Their smaller signal-to-noise ratios hampers their spectroscopic fittings, which is reflected in their higher associated uncertainties reported in Table 3.1. Nonetheless, for each plotted $\mathrm{K}$ group, there is a clear tendency for broader lines to present increasingly blueshifted velocities, as would be expected considering the results of $\$ 3.4 .1$ and $\$ 3.4 .2$. The same trend was also observed by Di Francesco et al. (2004) with $\mathrm{N}_{2} \mathrm{H}^{+}$lines toward the Ophiuchus A star-forming core.

Interestingly, the $\mathrm{K}=0$ lines are systematically broader and less blueshifted than the other groups, indicating that they might be tracing a slightly different region than the bulk of the emission of the other $\mathrm{K}$ transitions. To properly interpret this feature, observations with higher angular resolution are essential, but currently unavailable. Therefore, we can only provide a speculative discussion on a possible explanation to this plot: at low temperatures, the contributions from transitions with $K \neq 0$ to the rotational spectrum of $\mathrm{CH}_{3} \mathrm{CCH}$ are minor, and the emission is dominated by the $\mathrm{K}=0$ lines (see Figures 3.8 and 


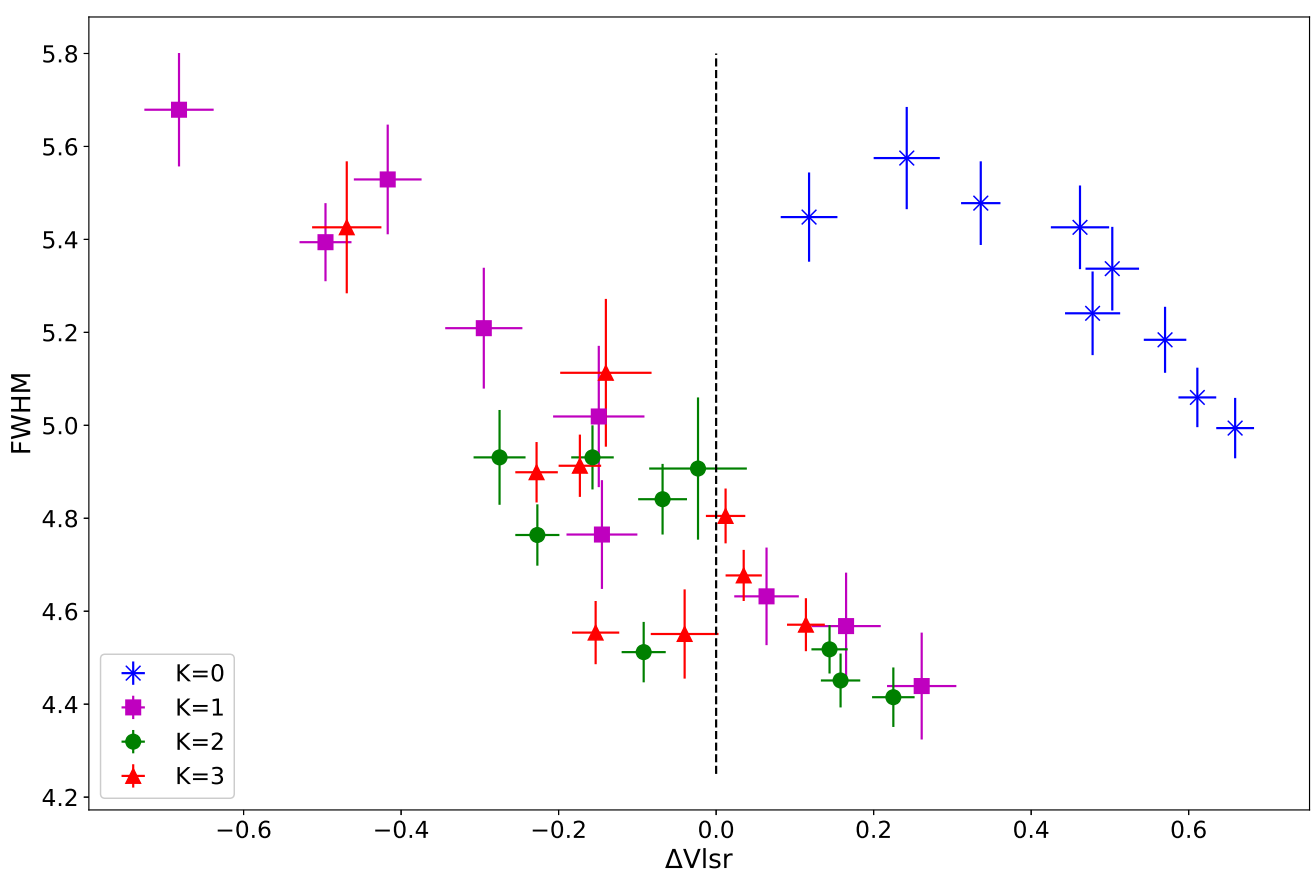

Figure 3.16: Line widths vs. peak velocities obtained from gaussian fittings of the observed transitions. Blue cross marks represent transitions with $K=0$, purple squares represent transitions with $K=1$, green circles represent transitions with $\mathrm{K}=2$ and red triangles represent transitions with $\mathrm{K}=3$. The black dashed line separates the blue- and red-shifted regions of the plot. G331 has a systemic velocity of $-90 \mathrm{~km} \mathrm{~s}^{-1}$.

3.9). As the temperature rises, however, the relative intensities of the $K \neq 0$ transitions rapidly increase, resulting in richer K-ladders. Thus, it is likely that the bulk of the $\mathrm{K}=0$ emission is originated from a cooler and more extended region, which does not contribute as significantly to the lines with $\mathrm{K} \neq 0$. These, in turn, must be tracing a hotter and more compact environment, resulting in a different kinematic signature. The systematic redshift of the peak velocities for the $\mathrm{K}=0$ lines compared the other groups also suggests interesting velocity features of the cold component.

In Figure 3.17, we illustrate the variation in the line widths and peak velocities by plotting the $\mathrm{K}=0$ and $\mathrm{K}=1$ transitions of the $\mathrm{J}=10-9$ and $\mathrm{J}=18-17 \mathrm{~K}$-ladders. The gaussian fittings show that lines with higher frequencies are broader, and the dash-dotted lines evince the negative correlation of the peak velocities with frequency. Further surveys of the $\mathrm{CH}_{3} \mathrm{CCH}$ emission toward G331 with higher angular resolution are therefore imperative to better understand the different gas components it might be tracing, as well as the "microphysics" of the hot core. 

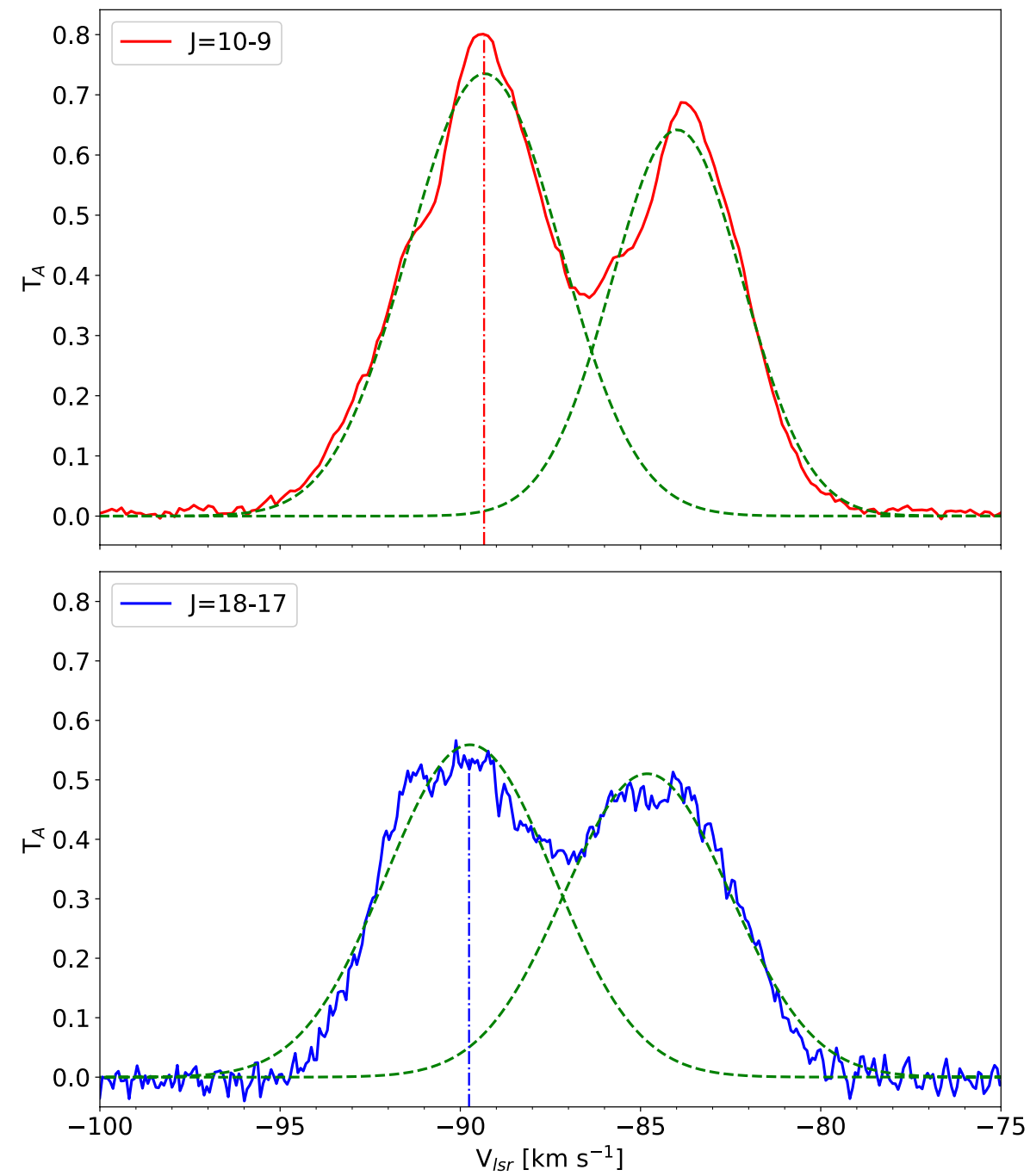

Figure 3.17: Top pannel: Spectrum of G331 showing the $10_{0}-9_{0}$ and $10_{1}-9_{1}$ transitions of $\mathrm{CH}_{3} \mathrm{CCH}$ in solid red line. Dashed green lines are the Gaussian profile fittings. The red dash-dot line indicates the peak velocity of the $10_{0}-9_{0}$ transition. Bottom pannel: Same as the top pannel, but for the $18_{0}-17_{0}$ and $18_{1}-17_{1}$ transitions - in blue instead of red. 
Chapter 4

\section{Conclusions and future perspectives}

\subsection{Conclusions}

In the present master's thesis, we have conducted a spectral survey of methyl acetylene $\left(\mathrm{CH}_{3} \mathrm{CCH}\right)$ toward the Hot Molecular Core G331.512-0.103, using the APEX $12 \mathrm{~m}$ telescope. We observed a total of 41 clear lines of $\mathrm{CH}_{3} \mathrm{CCH}$ above the detection limit of $3 \sigma$, without any contamination. To the best of our knowledge, the number of different lines of $\mathrm{CH}_{3} \mathrm{CCH}$ detected in this work was unprecedented, making this project the first spectral survey of methyl acetylene, and resulting in interesting insights on the physics of the source.

To investigate the excitation conditions of the $\mathrm{CH}_{3} \mathrm{CCH}$ emission and their implications on the physical properties of G331, we constructed rotational diagrams using all of the 41 detected lines. This analysis allows to estimate the excitation temperature $\left(\mathrm{T}_{\text {exc }}\right)$ and total column density $\left(\mathrm{N}_{t o t}\right)$ of the species under consideration, provided that the emission is under Local Thermodynamic Equilibrium (LTE). Since the observations were performed with a single-dish facility, the source size cannot be directly inferred from our data. Nonetheless, we explored both possible limiting scenarios of a compact $\left(\sim 5^{\prime \prime}\right)$ or an extended $\left(\sim 10^{\prime \prime}\right)$ emission. Opacity corrections were also considered in this analysis.

Synthetic LTE spectra of $\mathrm{CH}_{3} \mathrm{CCH}$ were constructed based on the values of $\mathrm{T}_{\text {exc }}$ and $\mathrm{N}_{\text {tot }}$ derived from the rotational diagrams. Moreover, pure rotational spectra of $\mathrm{CH}_{3} \mathrm{CCH}$ were simulated at a wide range of temperatures, in order to investigate the temperature dependence of the K-ladders' spectral profile. Adaptations of the rotational diagram technique, including modified diagrams and multiple-component fittings, were employed to scrutinize the temperature profile of the source. Finally, spectroscopic properties derived 
from fitting single gaussian profiles to the lines were used to explore the kinematics of the emitting gas. This approach is common for single-dish observations, which lack the angular resolution to directly study the small-scale structure of the source.

The main findings and conclusions achieved by virtue of the aforementioned procedures are listed below:

- From the rotational diagram analysis, we estimated an excitation temperature of $\mathrm{T}_{e x c}=40.2 \pm 1.1 \mathrm{~K}$ and a total column density of $\mathrm{N}_{t o t}=(3.9 \pm 0.3) \times 10^{16} \mathrm{~cm}^{-2}$ for a compact source, including opacity corrections. Comparatively, we derived $\mathrm{T}_{\text {exc }}=47.1 \pm 1.2$ $\mathrm{K}$ and $\mathrm{N}_{t o t}=(6.9 \pm 0.5) \times 10^{15} \mathrm{~cm}^{-2}$ for an extended emission. Those values of $\mathrm{T}_{\text {exc }}$ indicate that the bulk of the $\mathrm{CH}_{3} \mathrm{CCH}$ emission is originated from a cool gas, which is in accordance with other observational works and is inconsistent with a source size of $\sim 5^{\prime \prime}$. Thus, we additionally infer that the emission should encompass an extended region.

- Adopting the $\mathrm{N}_{\text {tot }}$ value of $\mathrm{CH}_{3} \mathrm{CCH}$ estimated for a source size of $\sim 10^{\prime \prime}$, and assuming an $\mathrm{H}_{2}$ column density of $\sim(4.5-9.1) \times 10^{23} \mathrm{~cm}^{-2}$, we derived a $\mathrm{CH}_{3} \mathrm{CCH}$ abundance of $\mathrm{X}\left(\mathrm{CH}_{3} \mathrm{CCH}\right)=\mathrm{N}_{\text {tot }}\left(\mathrm{CH}_{3} \mathrm{CCH}\right) / \mathrm{N}_{\text {tot }}\left(\mathrm{H}_{2}\right) \sim(1.5-7.6) \times 10^{-8}$. This value agrees with other observational works of $\mathrm{CH}_{3} \mathrm{CCH}$ toward star-forming regions.

- The synthetic LTE spectra based on the $\mathrm{T}_{e x c}$ and $\mathrm{N}_{t o t}$ values derived from the rotational diagrams become gradually less accurate for higher frequency transitions. Moreover, the $\mathrm{K}=2 / \mathrm{K}=3$ line intensity ratios of transitions within a given $\mathrm{K}$-ladder are strongly negatively correlated with $\mathrm{J}_{u}$, which is not predicted by the LTE models. Those observations firmly imply that the emission cannot be described by only one temperature component. Rather, we suggest that it is arising from a region with a temperature profile. Modified rotational diagrams with the abscissa plotted in logarithmic scale yield a better fit than the traditional diagram, which reinforces the hypothesis of a non-isothermal emission. Additionally, an analysis of the rotational diagram separated into two subsections provides upper and lower limits of, respectively, $\sim 60$ and $\sim 40 \mathrm{~K}$ for the temperature gradient. This approach enables us to assess the small-scale structure of the source with data from single-dish facilities, further broadening the temperature-probing capabilities of symmetric tops with three equivalent hydrogens - such as $\mathrm{CH}_{3} \mathrm{CCH}$. For a thorough analysis of the 
temperature profile of the source, however, it is imperative to observe a wide range of $\mathrm{CH}_{3} \mathrm{CCH}$ lines with high angular resolution, by means of interferometry instruments such as ALMA.

- The line widths and peak velocities of transitions with the same K quantum number are strongly correlated to their rest frequencies: for higher J quantum numbers, the lines become gradually broader and more blueshifted. Those trends indicate that the $\mathrm{CH}_{3} \mathrm{CCH}$ lines are indeed tracing different components of the gas, and that the warmer region - associated with higher-frequency transitions - is likely under stronger turbulence effects.

- By comparing the correlation between the line widths and the peak velocities, we observe that the $K=0$ transitions present a different kinematic signature than the rest of the lines. Thus, these transitions seem to be tracing a slightly different region than the bulk of the $K \neq 0$ lines: a potentially cooler and more extended gas. Surveys of this species with higher angular resolution are also fundamental to further explore the origin of this differentiation.

\subsection{Future perspectives}

A forthcoming paper on the research that was described in this thesis is currently under preparation. We expect to submit it to a peer-review journal on astrophysics by the first trimester of 2021 .

As was constantly mentioned in the previous section and throughout $\$ 3$, higher angular resolution is necessary to properly explore our conclusions. For this reason, the conduction of a spectral survey of $\mathrm{CH}_{3} \mathrm{CCH}$ toward G331 using interferometry instruments is paramount. Observations at higher frequencies are also desired in order to verify the intensity behavior of the $\mathrm{K}=2$ peaks, and possibly constrain the temperature profile. Accordingly, we plan to submit an observing proposal to ALMA in the near future, hopefully encompassing an even broader frequency range than the current work, and possibly including other promising HMCs additionally to G331.

Moreover, theoretical models of the time-dependent chemical abundances in star-forming regions are extremely useful to explore their evolution. By comparing the theoretical and 
observed abundances of different molecular species, those models allow to estimate the source's evolutionary stage, providing key information to put together the puzzle-pieces of massive star-formation. Thus, another future perspective of this work is to investigate the chemical network of $\mathrm{CH}_{3} \mathrm{CCH}$ in G331. By comparing its observed abundances to related species, such as $\mathrm{CCH}$, we aim to further our knowledge on the age of this source, and hopefully expand those findings to other young star-forming sites. 


\section{Bibliography}

Abgrall, H., Roueff, E., and Drira, I. (2000). Total transition probability and spontaneous radiative dissociation of $\mathrm{B}, \mathrm{C}, \mathrm{B}^{\prime}$ and $\mathrm{D}$ states of molecular hydrogen. AEAS $141: 297-$ 300.

Acharyya, K. and Herbst, E. (2017). Gas-grain Fluorine and Chlorine Chemistry in the Interstellar Medium. ApJ, 850(1):105.

Andre, P., Ward-Thompson, D., and Barsony, M. (2000). From Prestellar Cores to Protostars: the Initial Conditions of Star Formation. In Mannings, V., Boss, A. P., and Russell, S. S., editors, Protostars and Planets IV, page 59.

Andron, I., Gratier, P., Majumdar, L., Vidal, T. H. G., Coutens, A., Loison, J.-C., and Wakelam, V. (2018). Methyl cyanide $\left(\mathrm{CH}_{3} \mathrm{CN}\right)$ and propyne $\left(\mathrm{CH}_{3} \mathrm{CCH}\right)$ in the low-mass protostar IRAS 16293-2422. MNRAS, 481(4):5651-5659.

Belitsky, V., Lapkin, I., Fredrixon, M., Meledin, D., Sundin, E., Billade, B., Ferm, S. E., Pavolotsky, A., Rashid, H., Strandberg, M., Desmaris, V., Ermakov, A., Krause, S., Olberg, M., Aghdam, P., Shafiee, S., Bergman, P., De Beck, E., Olofsson, H., Conway, J., De Breuck, C., Immer, K., Yagoubov, P., Montenegro-Montes, F. M., Torstensson, K., Pérez-Beaupuits, J. P., Klein, T., Boland, W., Baryshev, A. M., Hesper, R., Barkhof, J., Adema, J., Bekema, M. E., and Koops, A. (2018). SEPIA - a new single pixel receiver at the APEX telescope. A\&GA, 612:A23.

Belloche, A., Müller, H. S. P., Menten, K. M., Schilke, P., and Comito, C. (2013). Complex organic molecules in the interstellar medium: IRAM $30 \mathrm{~m}$ line survey of Sagittarius B2(N) and (M). A\&̈A, 559:A47. 
Bergin, E. A., Goldsmith, P. F., Snell, R. L., and Ungerechts, H. (1994). CH 3C 2H as a Temperature Probe in Dense Giant Molecular Cloud Cores. ApJ, 431:674.

Beuther, H., Zhang, Q., Greenhill, L. J., Reid, M. J., Wilner, D., Keto, E., Shinnaga, H., Ho, P. T. P., Moran, J. M., Liu, S. Y., and Chang, C. M. (2005). Line Imaging of Orion KL at $865 \mu \mathrm{m}$ with the Submillimeter Array. ApJ, 632(1):355-370.

Bisschop, S. E., Jørgensen, J. K., van Dishoeck, E. F., and de Wachter, E. B. M. (2007). Testing grain-surface chemistry in massive hot-core regions. A $\& A, 465(3): 913-929$.

Blake, G. A., Sutton, E. C., Masson, C. R., and Phillips, T. G. (1987). Molecular Abundances in OMC-1: The Chemical Composition of Interstellar Molecular Clouds and the Influence of Massive Star Formation. ApJ, 315:621.

Bøgelund, E. G., Barr, A. G., Taquet, V., Ligterink, N. F. W., Persson, M. V., Hogerheijde, M. R., and van Dishoeck, E. F. (2019). Molecular complexity on disc scales uncovered by ALMA. Chemical composition of the high-mass protostar AFGL 4176. A\&GA, 628:A2.

Bonnell, I. A. and Bate, M. R. (2002). Accretion in stellar clusters and the collisional formation of massive stars. MNRAS, 336(2):659-669.

Bonnell, I. A., Bate, M. R., Clarke, C. J., and Pringle, J. E. (2001). Competitive accretion in embedded stellar clusters. MNRAS, 323(4):785-794.

Bonnell, I. A., Bate, M. R., and Zinnecker, H. (1998). On the formation of massive stars. MNRAS, 298(1):93-102.

Bottinelli, S., Ceccarelli, C., Neri, R., Williams, J. P., Caux, E., Cazaux, S., Lefloch, B., Maret, S., and Tielens, A. G. G. M. (2004). Near-Arcsecond Resolution Observations of the Hot Corino of the Solar-Type Protostar IRAS 16293-2422. ApJ, 617(1):L69-L72.

Brogan, C. L., Hunter, T. R., Cyganowski, C. J., Chandler, C. J., Friesen, R., and Indebetouw, R. (2016). The Massive Protostellar Cluster NGC 6334I at 220 au Resolution: Discovery of Further Multiplicity, Diversity, and a Hot Multi-core. ApJ, 832(2):187.

Bronfman, L., Alvarez, H., Cohen, R. S., and Thaddeus, P. (1989). A Deep CO Survey of Molecular Clouds in the Southern Milky Way. ApJS, 71:481. 
Bronfman, L., Casassus, S., May, J., and Nyman, L. A. (2000). The radial distribution of OB star formation in the Galaxy. A\&A, 358:521-534.

Bronfman, L., Garay, G., Merello, M., Mardones, D., May, J., Brooks, K. J., Nyman, L.-A., and Güsten, R. (2008). Discovery of an Extremely High Velocity, Massive, and Compact Molecular Outflow in Norma. ApJ, 672(1):391-397.

Buhl, D. and Snyder, L. E. (1973). The Detection of MM-Wave Transition of Methlacetylene. In Gordon, M. A. and Snyder, L. E., editors, Molecules in the Galactic Environment, page 187.

Burgdorf, M., Orton, G., van Cleve, J., Meadows, V., and Houck, J. (2006). Detection of new hydrocarbons in Uranus' atmosphere by infrared spectroscopy. Icarus, 184(2):634637.

Calcutt, H., Willis, E. R., Jørgensen, J. K., Bjerkeli, P., Ligterink, N. F. W., Coutens, A., Müller, H. S. P., Garrod, R. T., Wampfler, S. F., and Drozdovskaya, M. N. (2019). The ALMA-PILS survey: propyne $\left(\mathrm{CH}_{3} \mathrm{CCH}\right)$ in IRAS 16293-2422. A\&A, 631:A137.

Caselli, P. and Myers, P. C. (1995). The Line Width-Size Relation in Massive Cloud Cores. ApJ, 446:665.

Caswell, J. L. and Haynes, R. F. (1987). Southern HII regions : an extensive study of radio recombination lineemission. $A \mathscr{E} A, 171: 261-276$.

Cazaux, S., Tielens, A. G. G. M., Ceccarelli, C., Castets, A., Wakelam, V., Caux, E., Parise, B., and Teyssier, D. (2003). The Hot Core around the Low-mass Protostar IRAS 16293-2422: Scoundrels Rule! ApJ, 593(1):L51-L55.

Ceccarelli, C. (2005). Observations of low-mass protostars: Cold envelopes and hot corinos. Proceedings of the International Astronomical Union, 1(S231):1-16.

Cerqueira, H. B. A., Santos, J. C., Fantuzzi, F., Ribeiro, F. d. A., Rocco, M. L. M., Oliveira, R. R., and Rocha, A. B. (2020). Structure, stability, and spectroscopic properties of small acetonitrile cation clusters. The Journal of Physical Chemistry A, 124(34):6845-6855. PMID: 32702984. 
Cesaroni, R. (2005). Hot molecular cores. In Cesaroni, R., Felli, M., Churchwell, E., and Walmsley, M., editors, Massive Star Birth: A Crossroads of Astrophysics, volume 227, pages 59-69.

Cesaroni, R., Felli, M., Testi, L., Walmsley, C. M., and Olmi, L. (1997). The disk-outflow system around the high-mass (proto)star IRAS 20126+4104. A\& A, 325:725-744.

Cesaroni, R., Hofner, P., Walmsley, C. M., and Churchwell, E. (1998). Sub-arcsecond structure of hot cores in the NH_3 (4,4) line. A\&A, 331:709-725.

Cesaroni, R., Walmsley, C. M., Koempe, C., and Churchwell, E. (1991). Molecular clumps associated with ultra compact HII regions. A\&A, 252:278.

Churchwell, E. and Hollis, J. M. (1983). The kinetic temperature and CH3CCH column density profiles in SGR B2,Orion and DR 21. ApJ, 272:591-608.

Comito, C., Schilke, P., Phillips, T. G., Lis, D. C., Motte, F., and Mehringer, D. (2005). A Molecular Line Survey of Orion KL in the 350 Micron Band. ApJS, 156(2):127-167.

Cordiner, M. A., Palmer, M. Y., Nixon, C. A., Irwin, P. G. J., Teanby, N. A., Charnley, S. B., Mumma, M. J., Kisiel, Z., Serigano, J., Kuan, Y. J., Chuang, Y. L., and Wang, K. S. (2015). Ethyl Cyanide On Titan: Spectroscopic Detection and Mapping Using Alma. ApJ, 800(1):L14.

de Graauw, T., Feuchtgruber, H., Bezard, B., Drossart, P., Encrenaz, T., Beintema, D. A., Griffin, M., Heras, A., Kessler, M., Leech, K., Lellouch, E., Morris, P., Roelfsema, P. R., Roos-Serote, M., Salama, A., Vandenbussche, B., Valentijn, E. A., Davis, G. R., and Naylor, D. A. (1997). First results of ISO-SWS observations of Saturn: detection of CO_2_, CH_3_C_2_H, C_4_H_2_ and tropospheric H_2_O. A $\& A$ A, 321:L13-L16.

Di Francesco, J., André, P., and Myers, P. C. (2004). Quiescent Dense Gas in Protostellar Clusters: The Ophiuchus A Core. ApJ, 617(1):425-438.

Draine, B. T. (2011). Physics of the Interstellar and Intergalactic Medium. Princeton University Press. 
Dubrulle, A., Boucher, D., Burie, J., and Demaison, J. (1978). Microwave spectra of propyne and its [13c] isotopic species: Refined molecular structure of propyne. Journal of Molecular Spectroscopy, 72(1):158 - 164.

Dumke, M. and Mac-Auliffe, F. (2010). The APEX calibration plan: goals, implementation, and achievements. In Observatory Operations: Strategies, Processes, and Systems III, volume 7737 of Proc. SPIE, page 77371J.

Duronea, N. U., Bronfman, L., Mendoza, E., Merello, M., Finger, R., Reyes, N., HervíasCaimapo, C., Faure, A., Cappa, C. E., Arnal, E. M., Lépine, J. R. D., Kleiner, I., and Nyman, L. A. (2019). Cyanoacetylene in the outflow/hot molecular core G331.512-0.103. MNRAS, 489(2):1519-1532.

Edgar, R. (2004). A review of Bondi-Hoyle-Lyttleton accretion. New A Rev., 48(10):843859.

Endres, C. P., Schlemmer, S., Schilke, P., Stutzki, J., and Müller, H. S. P. (2016). The Cologne Database for Molecular Spectroscopy, CDMS, in the Virtual Atomic and Molecular Data Centre, VAMDC. Journal of Molecular Spectroscopy, 327:95-104.

Fontani, F., Cesaroni, R., Caselli, P., and Olmi, L. (2002). The structure of molecular clumps around high-mass young stellar objects. A\&A, 389:603-617.

Fouchet, T., Lellouch, E., Bézard, B., Feuchtgruber, H., Drossart, P., and Encrenaz, T. (2000). Jupiter's hydrocarbons observed with ISO-SWS: vertical profiles of C_2H_6 and C_2H_2, detection of CH_3C_2H. A\&SA, 355:L13-L17.

Galametz, M., Zhang, Z. Y., Immer, K., Humphreys, E., Aladro, R., De Breuck, C., Ginsburg, A., Madden, S. C., Møller, P., and Arumugam, V. (2016). Water, methanol and dense gas tracers in the local ULIRG Arp 220: results from the new SEPIA Band 5 Science Verification campaign. MNRAS, 462(1):L36-L40.

Galván-Madrid, R., Liu, H. B., Zhang, Z. Y., Pineda, J. E., Peng, T. C., Zhang, Q., Keto, E. R., Ho, P. T. P., Rodríguez, L. F., Zapata, L., Peters, T., and De Pree, C. G. (2013). MUSCLE W49: A Multi-Scale Continuum and Line Exploration of the Most Luminous Star Formation Region in the Milky Way. I. Data and the Mass Structure of the Giant Molecular Cloud. ApJ, 779(2):121. 
Garay, G. and Lizano, S. (1999). Massive Stars: Their Environment and Formation. PASP, 111(763):1049-1087.

García, P., Bronfman, L., Nyman, L.-Å., Dame, T. M., and Luna, A. (2014). Giant Molecular Clouds and Massive Star Formation in the Southern Milky Way. ApJS, $212(1): 2$.

Garrod, R. T. and Herbst, E. (2006). Formation of methyl formate and other organic species in the warm-up phase of hot molecular cores. A\&A, 457(3):927-936.

Genzel, R. (1991). Physical conditions and heating/cooling processes in high mass star formation regions. In Lada, C. J. and Kylafis, N. D., editors, The Physics of Star Formation and Early Stellar Evolution. Springer Netherlands.

Giannetti, A., Leurini, S., Wyrowski, F., Urquhart, J., Csengeri, T., Menten, K. M., König, C., and Güsten, R. (2017). ATLASGAL-selected massive clumps in the inner Galaxy. V. Temperature structure and evolution. A\&A, 603:A33.

Gibb, A. G., Wyrowski, F., and Mundy, L. G. (2004). High-Velocity Gas toward Hot Molecular Cores: Evidence for Collimated Outflows from Embedded Sources. ApJ, 616(1):301-318.

Goldsmith, P. F. and Langer, W. D. (1999). Population Diagram Analysis of Molecular Line Emission. ApJ, 517(1):209-225.

Gomez, Y., Garay, G., and Lizano, S. (1995). Warm Molecular Gas Associated with Cometary H II Regions. ApJ, 453:727.

Gordy, W. and Cook, R. L. (1984). Microwave molecular spectra. John Wiley \& Sons.

Güsten, R., Nyman, L. Å., Schilke, P., Menten, K., Cesarsky, C., and Booth, R. (2006). The Atacama Pathfinder EXperiment (APEX) - a new submillimeter facility for southern skies -. AESA, 454:L13-L16.

Guzmán, A. E., Guzmán, V. V., Garay, G., Bronfman, L., and Hechenleitner, F. (2018). Chemistry of the High-mass Protostellar Molecular Clump IRAS 16562-3959. ApJS, $236(2): 45$. 
Hatchell, J., Fuller, G. A., Millar, T. J., Thompson, M. A., and Macdonald, G. H. (2000). SCUBA imaging of high mass star formation regions. A\&A, 357:637-650.

Heaton, B. D., Little, L. T., and Bishop, I. S. (1989). THE "ultracompact hot core" of G $34.3+0.15$ : arcsecond resolution ammonia observations. A\&A, 213:148-154.

Heays, A. N., Visser, R., Gredel, R., Ubachs, W., Lewis, B. R., Gibson, S. T., and van Dishoeck, E. F. (2014). Isotope selective photodissociation of $\mathrm{N}_{2}$ by the interstellar radiation field and cosmic rays. A\&A, 562:A61.

Herbst, E. and van Dishoeck, E. F. (2009). Complex Organic Interstellar Molecules. ARA\&A, 47(1):427-480.

Hervías-Caimapo, C., Merello, M., Bronfman, L., Åke-Nyman, L., Garay, G., Lo, N., Evans, Neal J., I., López-Calderón, C., and Mendoza, E. (2019). ALMA Observations of the Massive Molecular Outflow G331.512-0.103. II. Physical Properties, Kinematics, and Geometry Modeling. ApJ, 872(2):200.

Herzberg, G. (1945). Molecular Spectra And Molecular Structure. Volume II. D. Van Nostrand Company, Inc.

Hickson, K. M., Wakelam, V., and Loison, J.-C. (2016). Methylacetylene $\left(\mathrm{CH}_{3} \mathrm{CCH}\right)$ and propene $\left(\mathrm{C}_{3} \mathrm{H}_{6}\right)$ formation in cold dense clouds: A case of dust grain chemistry. Molecular Astrophysics, 3:1-9.

Hofner, P., Wyrowski, F., Walmsley, C. M., and Churchwell, E. (2000). A C ${ }^{17}$ O Survey toward Ultracompact H II Regions. ApJ, 536(1):393-405.

Hollas, M. (2004). Modern Spectroscopy. john Wiley \& Sons.

Hollis, J. M., Snyder, L. E., Blake, D. H., Lovas, F. J., Suenram, R. D., and Ulich, B. L. (1981). New interstellar molecular transitions in the 2 millimeter range. ApJ, 251:541548.

Hunter, T. R., Brogan, C. L., Cyganowski, C. J., and Young, K. H. (2014). Subarcsecond Imaging of the NGC 6334 I(N) Protocluster: Two Dozen Compact Sources and a Massive Disk Candidate. ApJ, 788(2):187. 
Keto, E. (2002). On the Evolution of Ultracompact H II Regions. ApJ, 580(2):980-986.

Keto, E. (2003). The Formation of Massive Stars by Accretion through Trapped Hypercompact H II Regions. ApJ, 599(2):1196-1206.

Krumholz, M. R. (2006). High Mass Star Formation by Gravitational Collapse of Massive Cores. arXiv e-prints, pages astro-ph/0607429.

Krumholz, M. R. and Bonnell, I. A. (2007). Models for the Formation of Massive Stars. arXiv e-prints, page arXiv:0712.0828.

Krumholz, M. R., Cunningham, A. J., Klein, R. I., and McKee, C. F. (2010). Radiation Feedback, Fragmentation, and the Environmental Dependence of the Initial Mass Function. ApJ, 713(2):1120-1133.

Krumholz, M. R., Klein, R. I., and McKee, C. F. (2011). Radiation-hydrodynamic Simulations of the Formation of Orion-like Star Clusters. I. Implications for the Origin of the Initial Mass Function. ApJ, 740(2):74.

Krumholz, M. R., McKee, C. F., and Klein, R. I. (2005). The formation of stars by gravitational collapse rather than competitive accretion. Nature, 438(7066):332-334.

Kuiper, R. and Yorke, H. W. (2013). On the Effects of Optically Thick Gas (Disks) around Massive Stars. ApJ, 763(2):104.

Kuiper, T. B. H., Kuiper, E. N. R., Dickinson, D. F., Turner, B. E., and Zuckerman, B. (1984). Methyl acetylene as a temperature probe for dense interstellar clouds. ApJ, 276:211-220.

Lada, C. J. and Kylafis, N. D. (1999). The Origin of Stars and Planetary Systems. Springer, Dordrecht.

Levine, I. N. (1975). Molecular Spectroscopy. John Wiley \& Sons.

Li, X., Heays, A. N., Visser, R., Ubachs, W., Lewis, B. R., Gibson, S. T., and van Dishoeck, E. F. (2013). Photodissociation of interstellar $\mathrm{N}_{2}$. A\& A, 555:A14.

Linnartz, H., Ioppolo, S., and Fedoseev, G. (2015). Atom addition reactions in interstellar ice analogues. International Reviews in Physical Chemistry, 34(2):205-237. 
Lovas, F. J. (2004). NIST Recommended Rest Frequencies for Observed Interstellar Molecular Microwave Transitions-2002 Revision. Journal of Physical and Chemical Reference Data, 33:177-355.

Lovas, F. J., Johnson, D. R., Buhl, D., and Snyder, L. E. (1976). Millimeter emission lines in Orion A. ApJ, 209:770-777.

Maret, S., Hily-Blant, P., Pety, J., Bardeau, S., and Reynier, E. (2011). Weeds: a CLASS extension for the analysis of millimeter and sub-millimeter spectral surveys. $A \& A$, 526:A47.

Mauersberger, R., Henkel, C., Walmsley, C. M., Sage, L. J., and Wiklind, T. (1991). Dense gas in nearby galaxies. V. Multilevel studies of $\mathrm{CH} 3 \mathrm{CCH}$ and $\mathrm{CH} 3 \mathrm{CN}$. A $\& A, 247: 307$.

Mauersberger, R., Wilson, T. L., and Henkel, C. (1988). A multilevel study of ammonia in a star-forming regions. IV. Emissionand absorption toward W3 (OH). AEA, 201:123-130.

McGuire, B. A. (2018). 2018 Census of Interstellar, Circumstellar, Extragalactic, Protoplanetary Disk, and Exoplanetary Molecules. ApJS, 239(2):17.

McKee, C. F. and Tan, J. C. (2003). The Formation of Massive Stars from Turbulent Cores. ApJ, 585(2):850-871.

McLaughlin, D. E. and Pudritz, R. E. (1997). Gravitational Collapse and Star Formation in Logotropic and Nonisothermal Spheres. ApJ, 476(2):750-765.

Mendoza, E., Bronfman, L., Duronea, N. U., Lépine, J. R. D., Finger, R., Merello, M., Hervías-Caimapo, C., Gama, D. R. G., Reyes, N., and Åke-Nyman, L. (2018). G331.5120.103: An Interstellar Laboratory for Molecular Synthesis. I. The Ortho-to-para Ratios for $\mathrm{CH}_{3} \mathrm{OH}$ and $\mathrm{CH}_{3} \mathrm{CN}$. ApJ, 853(2):152.

Merello, M., Bronfman, L., Garay, G., Lo, N., Evans, Neal J., I., Nyman, L.-A., Cortés, J. R., and Cunningham, M. R. (2013a). ALMA Observations of the Massive Molecular Outflow G331.512-0.103. ApJ, 774(1):L7.

Merello, M., Bronfman, L., Garay, G., Nyman, L.-Å., Evans, Neal J., I., and Walmsley, C. M. (2013b). Physical Characteristics of G331.5-0.1: The Luminous Central Region of a Giant Molecular Cloud. ApJ, 774(1):38. 
Millar, T. J. and Freeman, A. (1984). Chemical modelling of molecular sources - I. TMC-1. MNRAS, 207:405-423.

Millar, T. J. and Williams, D. A. (1988). Rate Coefficients in Astrochemistry. Proceedings of a Conference held in UMIST, Manchester, United Kingdom, September 21-24, 1987., volume 146. Springer Netherlands.

Milligan, T. A. (2005). Modern Antenna Design. John Wiley \& Sons.

Mills, I. M. (1972). Molecular Spectroscopy: Modern Research. Academic Press, Inc.

Molinari, S., Merello, M., Elia, D., Cesaroni, R., Testi, L., and Robitaille, T. (2016). Calibration of Evolutionary Diagnostics in High-mass Star Formation. ApJ, 826(1):L8.

Muenter, J. S. and Laurie, V. W. (1966). Deuterium isotope effects on molecular dipole moments by microwave spectroscopy. The Journal of Chemical Physics, 45(3):855-858.

Myers, P. C. and Fuller, G. A. (1992). Density Structure and Star Formation in Dense Cores with Thermal and Nonthermal Motions. ApJ, 396:631.

Nagy, Z., van der Tak, F. F. S., Fuller, G. A., and Plume, R. (2015). Physical and chemical differentiation of the luminous star-forming region W49A. Results from the JCMT Spectral Legacy Survey. A\&A, 577:A127.

Neufeld, D. A., Falgarone, E., Gerin, M., Godard, B., Herbst, E., Pineau des Forêts, G., Vasyunin, A. I., Güsten, R., Wiesemeyer, H., and Ricken, O. (2012). Discovery of interstellar mercapto radicals (SH) with the GREAT instrument on SOFIA. $A \mathscr{G} A$, 542:L6.

Nyman, L. Å., Lerner, M., Nielbock, M., Anciaux, M., Brooks, K., Chini, R., Albrecht, M., Lemke, R., Kreysa, E., Zylka, R., Johansson, L. E. B., Bronfman, L., Kontinen, S., Linz, H., and Stecklum, B. (2001). SIMBA explores the southern sky. The Messenger, 106:40-44.

Öberg, K. I., Boamah, M. D., Fayolle, E. C., Garrod, R. T., Cyganowski, C. J., and van der Tak, F. (2013). The Spatial Distribution of Organics toward the High-mass YSO NGC 7538 IRS9. ApJ, 771(2):95. 
Offner, S. S. R., Kratter, K. M., Matzner, C. D., Krumholz, M. R., and Klein, R. I. (2010). The Formation of Low-mass Binary Star Systems Via Turbulent Fragmentation. ApJ, $725(2): 1485-1494$.

Osorio, M., Lizano, S., and D'Alessio, P. (1999). Hot Molecular Cores and the Formation of Massive Stars. ApJ, 525(2):808-820.

Papousek, D. and Aliev, M. R. (1982). Molecular Vibrational Rotational Spectra. Elsevier.

Pickett, H. M., Poynter, R. L., Cohen, E. A., Delitsky, M. L., Pearson, J. C., and Müller, H. S. P. (1998). Submillimeter, millimeter and microwave spectral line catalog. J. Quant. Spec. Radiat. Transf., 60:883-890.

Plume, R., Jaffe, D. T., Evans, Neal J., I., Martín-Pintado, J., and Gómez-González, J. (1997). Dense Gas and Star Formation: Characteristics of Cloud Cores Associated with Water Masers. ApJ, 476(2):730-749.

Puzzarini, C. (2017). Astronomical complex organic molecules: Quantum chemistry meets rotational spectroscopy. International Journal of Quantum Chemistry, 117(2):129-138.

Puzzarini, C. and Barone, V. (2020). The challenging playground of astrochemistry: an integrated rotational spectroscopy - quantum chemistry strategy. Physical Chemistry Chemical Physics (Incorporating Faraday Transactions), 22(12):6507-6523.

Puzzarini, C., Stanton, J. F., and Gauss, J. (2010). Quantum-chemical calculation of spectroscopic parameters for rotational spectroscopy. International Reviews in Physical Chemistry, 29(2):273-367.

Qasim, D., Fedoseev, G., Chuang, K. J., He, J., Ioppolo, S., van Dishoeck, E. F., and Linnartz, H. (2020). An experimental study of the surface formation of methane in interstellar molecular clouds. Nature Astronomy, 4:781-785.

Qasim, D., Fedoseev, G., Lamberts, T., Chuang, K.-J., He, J., Ioppolo, S., Kästner, J., and Linnartz, H. (2019). Alcohols on the Rocks: Solid-State Formation in a H3CC $\equiv \mathrm{CH}+$ OH Cocktail under Dark Cloud Conditions. ACS Earth and Space Chemistry, 3(6):986999. 
Qiu, J., Zhang, J., Zhang, Y., Jia, L., and Tang, X. (2020). $\lambda=2 \mathrm{~mm}$ spectroscopy observations toward the circumnuclear disk of NGC 1068. A\&A, 634:A125.

Qiu, K., Zhang, Q., Megeath, S. T., Gutermuth, R. A., Beuther, H., Shepherd, D. S., Sridharan, T. K., Testi, L., and De Pree, C. G. (2008). Spitzer IRAC and MIPS Imaging of Clusters and Outflows in Nine High-Mass Star Forming Regions. ApJ, 685(2):10051025.

Roberts, H., van der Tak, F. F. S., Fuller, G. A., Plume, R., and Bayet, E. (2011). Dense molecular gas towards W49A: a template for extragalactic starbursts? A\& A, 525:A107.

Russeil, D., Schneider, N., Anderson, L. D., Zavagno, A., Molinari, S., Persi, P., Bontemps, S., Motte, F., Ossenkopf, V., André, P., Arzoumanian, D., Bernard, J. P., Deharveng, L., Didelon, P., Di Francesco, J., Elia, D., Hennemann, M., Hill, T., Könyves, V., Li, J. Z., Martin, P. G., Nguyen Luong, Q., Peretto, N., Pezzuto, S., Polychroni, D., Roussel, H., Rygl, K. L. J., Spinoglio, L., Testi, L., Tigé, J., Vavrek, R., Ward-Thompson, D., and White, G. (2013). The Herschel view of the massive star-forming region NGC 6334 . $A \& A, 554: \mathrm{A} 42$.

Rybicki, G. B. and Lightman, A. P. (1985). Radiative Processes in Astrophysics. Wiley$\mathrm{VCH}$.

Sadaghiani, M., Sánchez-Monge, Á., Schilke, P., Liu, H. B., Clarke, S. D., Zhang, Q., Girart, J. M., Seifried, D., Aghababaei, A., Li, H., Juárez, C., and Tang, K. S. (2020). Physical properties of the star-forming clusters in NGC 6334. A study of the continuum dust emission with ALMA. AEAA, 635:A2.

Santos, J. C., Rocha, A. B., and Oliveira, R. R. (2020). Rotational spectrum simulations of asymmetric tops in an astrochemical context. Journal of Molecular Modeling, 26(278).

Schiff, H. I. and Bohme, D. K. (1979). An ion-molecule scheme for the synthesis of hydrocarbon-chain and organonitrogen molecules in dense interstellar clouds. ApJ, 232:740-746.

Schmidt, D. R. and Ziurys, L. M. (2019). Exotic Carbon Chemistry in a Planetary Nebula: The Unusual Case of K4-47. ApJ, 881(2):L38. 
Shu, F. H. (1977). Self-similar collapse of isothermal spheres and star formation. ApJ, 214:488-497.

Shu, F. H., Adams, F. C., and Lizano, S. (1987). Star formation in molecular clouds: observation and theory. ARAËA, 25:23-81.

Silva, A., Zhang, Q., Sanhueza, P., Lu, X., Beltran, M. T., Fallscheer, C., Beuther, H., Sridharan, T. K., and Cesaroni, R. (2017). SMA Observations of the Hot Molecular Core IRAS 18566+0408. ApJ, 847(2):87.

Stahler, S. W., Shu, F. H., and Taam, R. E. (1980). The evolution of protostars. I - Global formulation and results. ApJ, 241:637-654.

Tan, J. C. (2018). Fire from Ice - Massive Star Birth from Infrared Dark Clouds. IAU Symposium, 332:139-152.

Tan, J. C., Beltrán, M. T., Caselli, P., Fontani, F., Fuente, A., Krumholz, M. R., McKee, C. F., and Stolte, A. (2014). Massive Star Formation. In Beuther, H., Klessen, R. S., Dullemond, C. P., and Henning, T., editors, Protostars and Planets VI, page 149.

Taniguchi, K., Herbst, E., Caselli, P., Paulive, A., Maffucci, D. M., and Saito, M. (2019). Cyanopolyyne Chemistry around Massive Young Stellar Objects. ApJ, 881(1):57.

Taniguchi, K., Saito, M., Majumdar, L., Shimoikura, T., Dobashi, K., Ozeki, H., Nakamura, F., Hirota, T., Minamidani, T., Miyamoto, Y., and Kaneko, H. (2018). Chemical Diversity in Three Massive Young Stellar Objects Associated with $6.7 \mathrm{GHz}^{\mathrm{CH}_{3} \mathrm{OH}}$ Masers. ApJ, 866(2):150.

Tielens, A. G. G. M. (2005). The Physics and Chemistry of the Interstellar Medium. Cambridge University Press.

Townes, C. H. and Schawlow, A. L. (1975). Microwave Spectroscopy. Dover Publications. Turner, B. E., Terzieva, R., and Herbst, E. (1999). The Physics and Chemistry of Small Translucent Molecular Clouds. XII. More Complex Species Explainable by Gas-Phase Processes. ApJ, 518(2):699-732.

van Dishoeck, E. F. (2014). Astrochemistry of dust, ice and gas: introduction and overview. Faraday Discussions, 168:9. 
van Dishoeck, E. F. and Black, J. H. (1988). The Photodissociation and Chemistry of Interstellar CO. ApJ, 334:771.

Vassilev, V., Meledin, D., Lapkin, I., Belitsky, V., Nyström, O., Henke, D., Pavolotsky, A., Monje, R., Risacher, C., Olberg, M., Strandberg, M., Sundin, E., Fredrixon, M., Ferm, S. E., Desmaris, V., Dochev, D., Pantaleev, M., Bergman, P., and Olofsson, H. (2008). A Swedish heterodyne facility instrument for the APEX telescope. $A \mathscr{E} A$, 490(3):1157-1163.

Vastel, C., Ceccarelli, C., Lefloch, B., and Bachiller, R. (2014). The Origin of Complex Organic Molecules in Prestellar Cores. ApJ, 795(1):L2.

Visser, R., van Dishoeck, E. F., Doty, S. D., and Dullemond, C. P. (2009). The chemical history of molecules in circumstellar disks. I. Ices. A\&A, 495(3):881-897.

Walmsley, C. M., Cesaroni, R., Olmi, L., Churchwell, E., and Hofner, P. (1995). The Massive Hot Core Associated with G31.41+0.31. Ap\&SS, 224(1-2):173-175.

Wang, P., Li, Z.-Y., Abel, T., and Nakamura, F. (2010). Outflow Feedback Regulated Massive Star Formation in Parsec-Scale Cluster-Forming Clumps. ApJ, 709(1):27-41.

Western, C. M. (2017). PGOPHER: A program for simulating rotational, vibrational and electronic spectra. J. Quant. Spec. Radiat. Transf., 186:221-242.

Williams, D. A. and Cecchi-Pestellini, C. (2016). The Chemistry of Cosmic Dust. The Royal Society of Chemistry.

Wilson, T., Rohlfs, K., and Huettemeister, S. (2009). Tools of Radio Astronomy. SpringerVerlag Berlin Heidelberg.

Wyrowski, F., Schilke, P., Walmsley, C. M., and Menten, K. M. (1999). Hot Gas and Dust in a Protostellar Cluster near W3(OH). ApJ, 514(1):L43-L46.

Zahorecz, S., Jimenez-Serra, I., Testi, L., Immer, K., Fontani, F., Caselli, P., Wang, K., and Toth, L. V. (2017). Gas versus solid-phase deuterated chemistry: HDCO and $\mathrm{D}_{2} \mathrm{CO}$ in massive star-forming regions. $A \mathscr{E} A, 602: \mathrm{L} 3$. 
Zhang, C.-P., Wang, J.-J., Xu, J.-L., Wyrowski, F., and Menten, K. M. (2014). Submillimeter Array and Very Large Array Observations in the Hypercompact H II Region G35.58-0.03. ApJ, 784(2):107.

Zhang, Q., Sridharan, T. K., Hunter, T. R., Chen, Y., Beuther, H., and Wyrowski, F. (2007). A jet-like outflow toward the high-mass (proto) stellar object IRAS 18566+0408. $A \mathscr{E} A, 470(1): 269-279$.

Zinnecker, H. and Yorke, H. W. (2007). Toward Understanding Massive Star Formation. ARA\&A, 45(1):481-563. 
Appendix 

Appendix A

\section{Total spectrum of G331}

A.1 Here we present the total spectrum of G331, including the observations conducted with the SEPIA180, APEX-1 and APEX-2 receivers of the APEX $12 \mathrm{~m}$ telescope. 

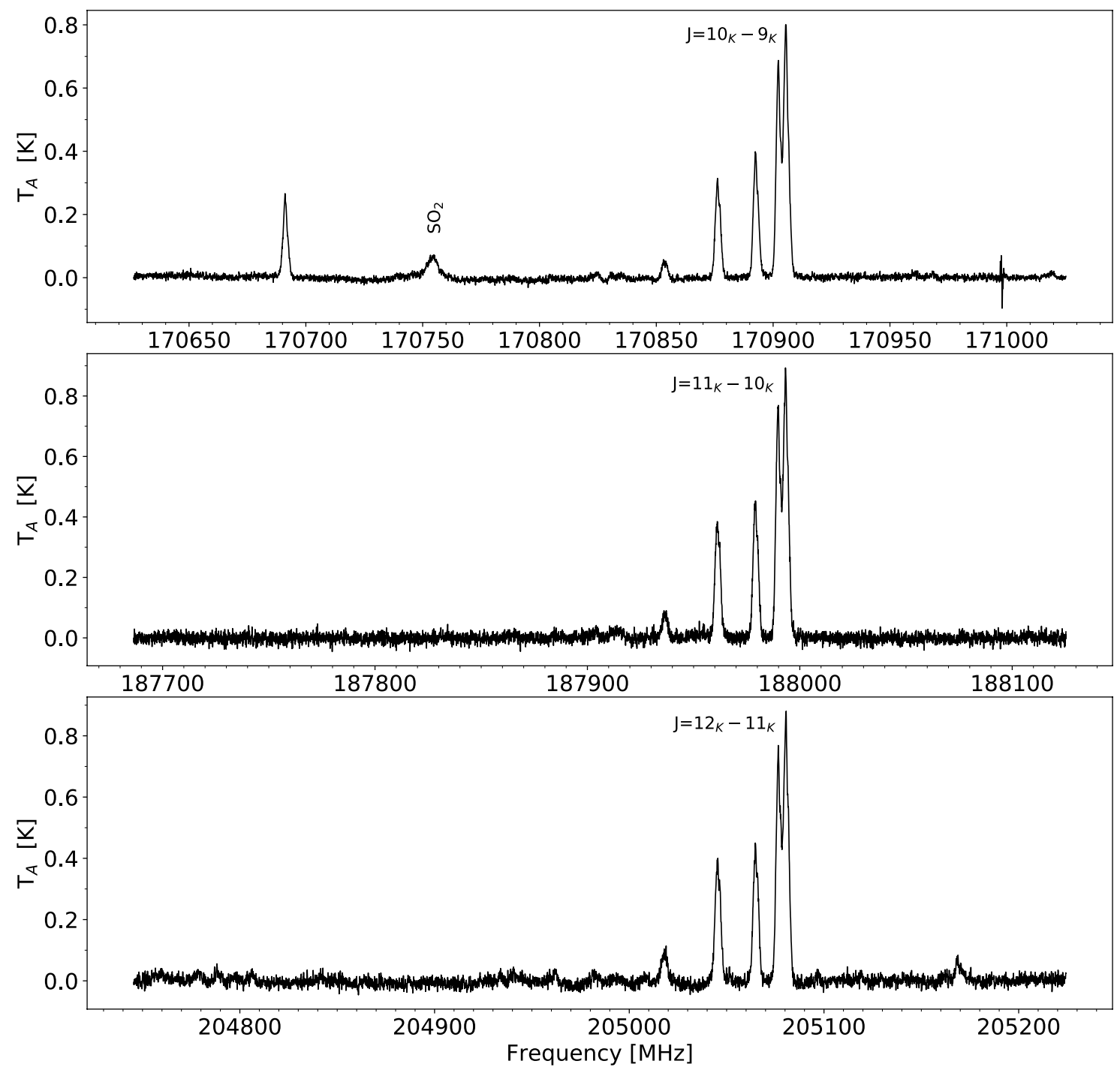

Figure A.1: Spectral bands: 170630-171030 MHz; 187690-188130 MHz; 204750-205220 MHz 

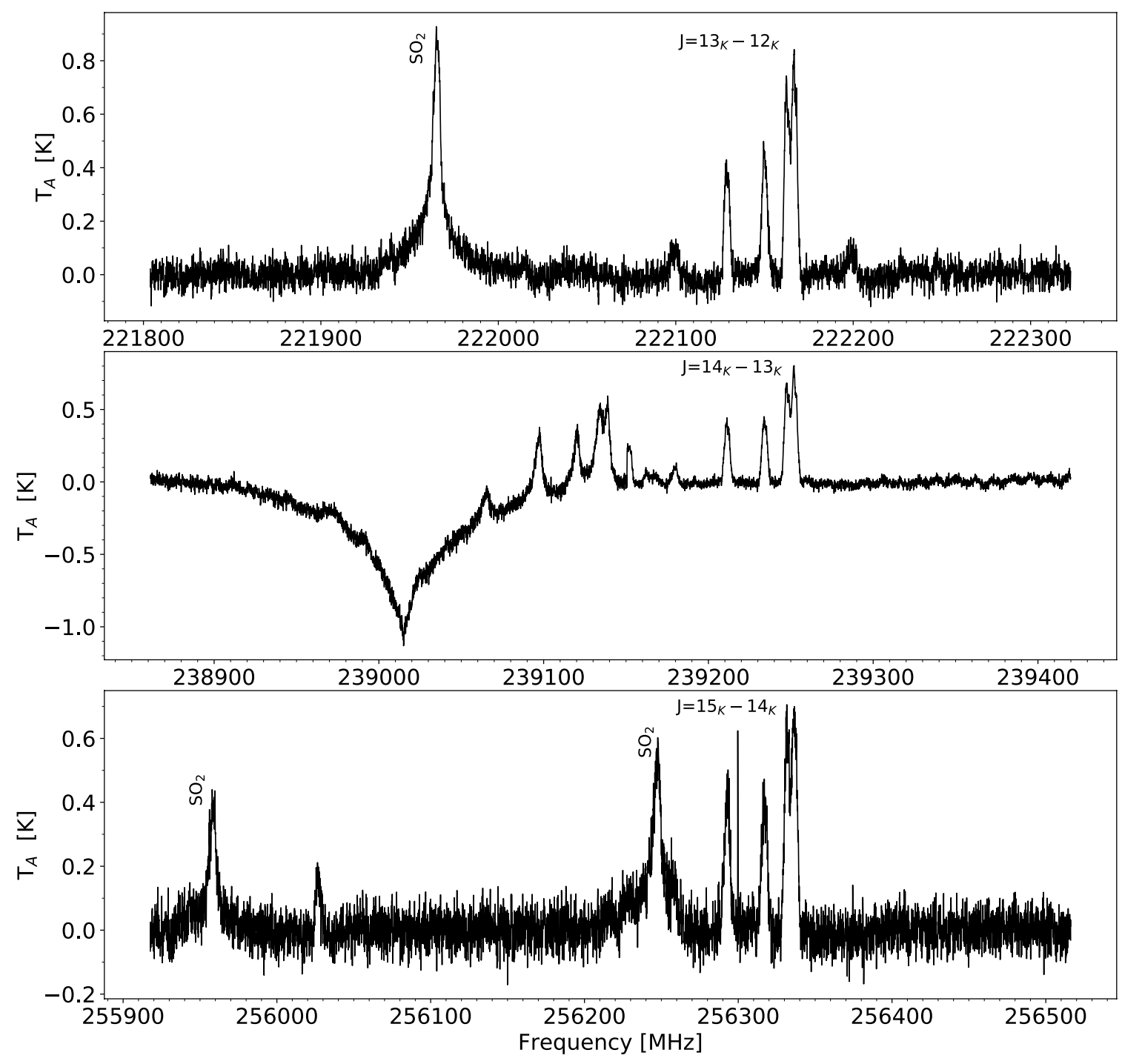

Figure A.2: Spectral bands: 221800-222320 MHz; 238840-239430 MHz; 255920-256520 MHz 

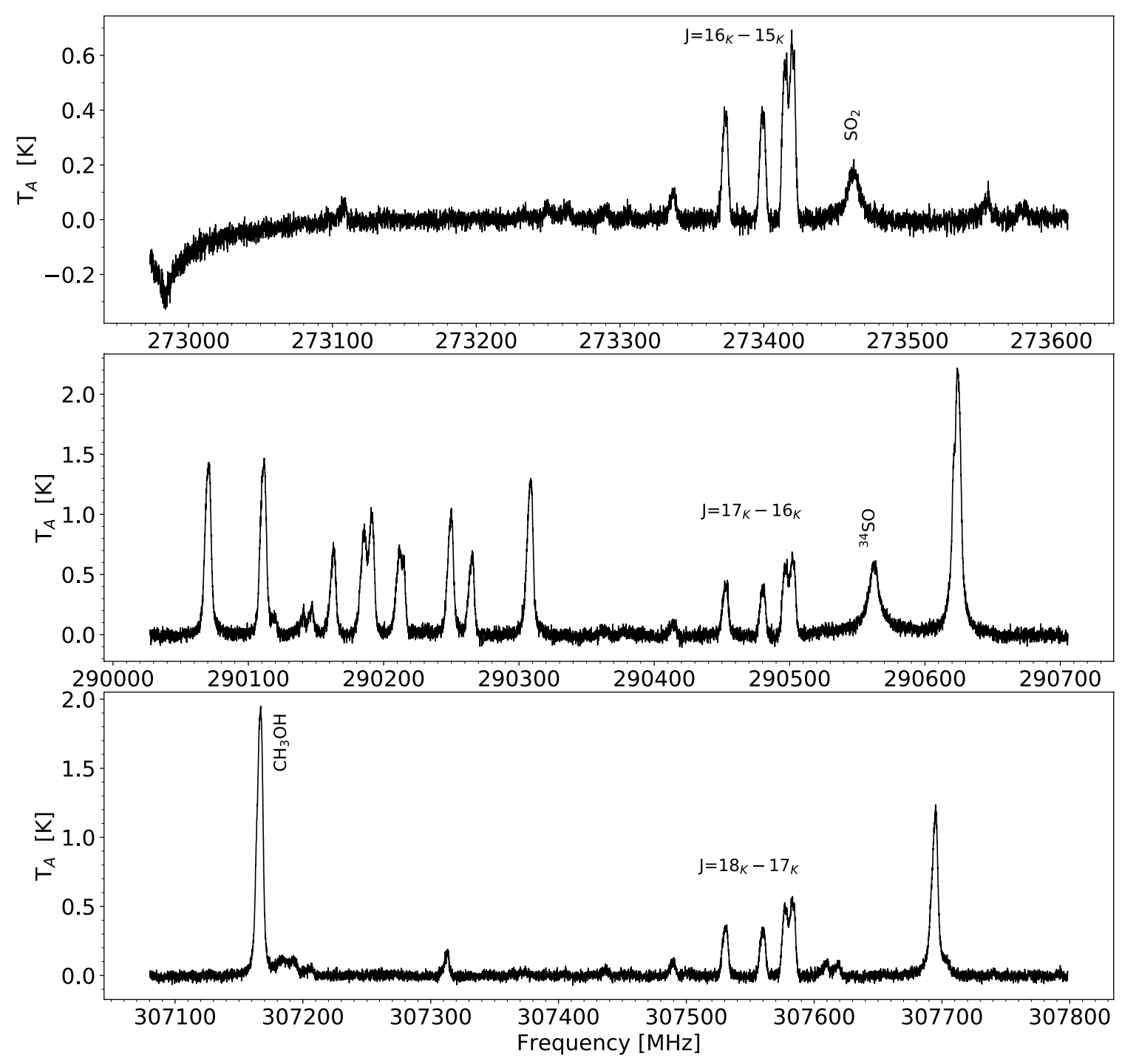

Figure A.3: Spectral bands: 272970-273610 MHz; 290030-290710 MHz; $\sim 307080-307800 \mathrm{MHz}$ 